\title{
The $\operatorname{Mn}(n, x \gamma)$ Reaction Cross Section for Incident Neutron Energies Between 0.2 and $20.0 \mathrm{MeV}$
}

\author{
G. L. Morgan
}

\section{NOTICE}

QRTIONS QF THIS REPORT ARE IILEGIBLE. It hes been reproduced foadest possiblo avail- 


\section{DISCLAIMER}

This report was prepared as an account of work sponsored by an agency of the United States Government. Neither the United States Government nor any agency Thereof, nor any of their employees, makes any warranty, express or implied, or assumes any legal liability or responsibility for the accuracy, completeness, or usefulness of any information, apparatus, product, or process disclosed, or represents that its use would not infringe privately owned rights. Reference herein to any specific commercial product, process, or service by trade name, trademark, manufacturer, or otherwise does not necessarily constitute or imply its endorsement, recommendation, or favoring by the United States Government or any agency thereof. The views and opinions of authors expressed herein do not necessarily state or reflect those of the United States Government or any agency thereof. 


\section{DISCLAIMER}

Portions of this document may be illegible in electronic image products. Images are produced from the best available original document. 
Printed in the United States of America. Available from National Technical Information Service

U.S. Department of Commerce

5285 Port Royal Road, Springfield, Virginia 22161

Price: Printed Copy \$4.50; Microfiche \$2.25

This report was prepared as an account of work sponsored by the United States Government. Neither the United States nor the Energy Research and Development Administration/United States Nuclear Regulatory Commission, nor any of their employees, nor any of their contractors, subcontractors, or their employees, makes any warranty, express or implied, or assumes any legal liability or responsibility for the accuracy, completeness or usefulness of any information, apparatus, product or process disclosed, or represents that its use would not infringe privately owned rights. 
ORNL/TM-5531

Contract No. W-7405-eng-26

Neutron Physics Division

THE $M n(n, x y)$ REACTION CROSS SECTION FOR INCIDENT

NEUTRON ENERGIES BETWEEN 0.2 AND $20.0 \mathrm{MeV}$

G. L. Morgan

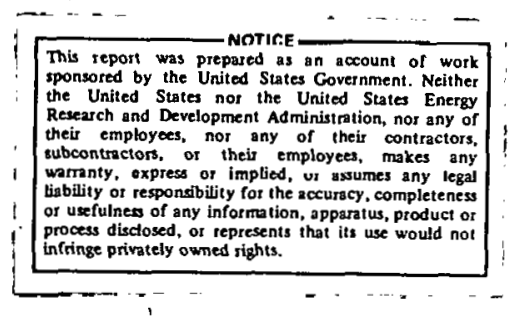

AUGUST 1976

OAK RIDGE NATIONAL LABORATORY

Oak Ridge, Tennessee 37830

operated by

UNION CARBIDE CORPORATION

for the

ENERGY RESEARCH AND DEVELOPMENT ADMINISTRATION

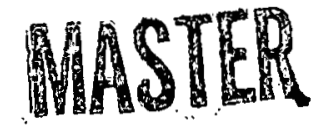




\section{THIS PAGE}

\section{WAS INTENTIONALLY LEFT BLANK}




\section{ABSTRACT}

Differential cross sections for the neutron-induced gamma-ray production from natural manganese have been measured for incident neutron energies between 0.2 and $20.0 \mathrm{MeV}$. The Oak Ridge Linear Accelerator (ORELA) was used to provide the neutrons and a $\mathrm{NaI}$ spectrometer to detect the gamma rays at $125^{\circ}$. The data presented are the double differential cross section, $d^{2} \sigma / d \Omega d E$, for gamma-ray energies between 0.22 and $10.6 \mathrm{MeV}$ for coarse intervals in incident neutron energy. The integrated yield of gamma rays of energies greater than $220 \mathrm{keV}$ with higher resolution in the neutron energy is also presented. The experimental results are compared with the Evaluated Neutron Data Files (ENDF). 


\section{INTRODUCTION}

As part of a continuing program ${ }^{1}$ for determining numerical values of gamma-ray production cross sections for neutron-induced reactions, we have measured the absolute differential cross sections for gamma rays produced by neutron interactions with natural manganese. The data are presented in this report in tabular and graphical form and are compared to the current evaluated data file for manganese (MAT 1197 ENDF/B-IV). ${ }^{2}$

Two methods of data analysis were employed. The first gives the detailed gamma-ray spectra for a series of relatively coarse intervals in incident neutron energy while the second method uses integral quantities to illustrate the detailed behavior of the cross sections as a function of the incident neutron energy. The second approach is used to facilitate comparison of the experimental and evaluated data in the region of the thresholds for the $(n, 2 n)$ and $(n, 3 n)$ reactions.

\section{EXPERIMENTAL PROCEDURE}

Details of the experimental procedure are given elsewhere ${ }^{3}$ and only a brief description will be given here. Neutrons were produced by photonuclear processes due to bremsstrahlung from the impact on a tantalum target of electrons from the Oak Ridge Electron Linear Accelerator (ORELA). The present experiment employed an electron beam energy of 135 $\mathrm{MeV}$ with a repetition rate of 800 pulses per second and a pulse width of $12 \mathrm{~ns}$. The total electron beam power was $18 \mathrm{~kW}$.

Neutrons produced at the linac target traversed a $47.35 \mathrm{~m}$ flight path and were incident on a thin slab of natural manganese oriented $45^{\circ}$ 
with respect to the incident beam. The slab was $30 \mathrm{~cm}$ wide by $30 \mathrm{~cm}$ high with a thickness of 0.0388 atoms/barn. Gamma rays originating in the sample were detected by a heavily shielded $12.5 \mathrm{~cm}$ by $12.5 \mathrm{~cm}$ NaI detector at $125^{\circ}$ with respect to the incident neutron beam. For each event in the detector data were recorded in a two-parameter array containing gamma-ray pulse height as a function of time-of-flight for the incident neutron.

The neutron flux at the sample position was determined in a separate experiment using calibrated thick organic scintillators. During the course of the gamma-ray measurements the flux was monitored using a small plastic scintillator in the edge of the neutron beam $30 \mathrm{~m}$ from the source.

\section{DATA REDUCTION}

Two methods of data reduction were employed. In the first, the pulse height spectra were integrated over intervals of neutron time-of-flight to form pulse-height spectra for specific incident neutron energy ranges. These intervals ranged in width from $0.5 \mathrm{MeV}$ at energies below $5 \mathrm{MeV}$ to $3 \mathrm{MeV}$ in the range 14 to $20 \mathrm{MeV}$. The spectra so formed were then unfolded using the code FERD and measured response functions of the NaI detector. The results were the gamma-ray spectra defined by 149 points covering the gamma-ray energy range from 0.22 to $10.6 \mathrm{MeV}$. After correction for selfabsorption and neutron self-shielding in the sample, these spectra were normalized to cross sections using the measured neutron flux and sample thickness. A further correction was then applied to the unfolded data. The contribution to the observed cross section at $E_{\gamma}=0.511 \mathrm{MeV}$ due to pair production in the sample was analytically removed. This was done 
by first calculating the pair production probability as a function of gamma-ray energy using a Monte Carlo technique. Implicit in the calculation are the assumptions of uniform gamma-ray production probability within the sample volume intercepted by the beam and the isotropy of emitted gamma rays. The total $0.511-\mathrm{MeV}$ cross section within each neutron energy group was then calculated from the product of the observed gammaray cross section and the pair production probability. This cross section was then "smeared" with the detector resolution and subtracted from the original data. The magnitude of the correction ranged from on the order of $0.05 \mathrm{mb} / \mathrm{sr}$ for $E_{n}=1.0-1.5 \mathrm{MeV}$ to $11 \mathrm{mb} / \mathrm{sr}$ for $E_{n}=10-12 \mathrm{MeV}$.

These results are presented in the first set of figures at the end of this report. Figure $l$ is a three-dimensional representation of the data giving cross section versus gamma-ray and incident neutron energy. Figures 2-20 present the detailed gamma-ray spectra for each incident neutron energy interval. These are compared to cross sections generated from the evaluation (ENDF/B-IV MAT 1197) by averaging over the appropriate neutron energy interval.

The data described above provide detailed information about the secondary gamma-ray spectra, but because the unfolding technique requires good statistical accuracy the data must be binned over large neutron energy intervals. Therefore, a second type of data reduction, pulseheight weighting, ${ }^{4,5}$ was also used. This technique provided only integral information about the secondary gamma spectra (e.g., total yield and average photon energy); but because the demands on statistical accuracy are less it allowed better resolution in the incident neutron energy. In this work the pulse height weighting analysis was applied to spectra 
formed by integration over time-of-flight intervals corresponding to $\Delta E_{n}=0.1 \mathrm{MeV}$ at $E_{n}=1 \mathrm{MeV}$ increasing to $\Delta E_{n}=1.0 \mathrm{MeV}$ at $E_{n}=20 \mathrm{MeV}$. The results of this analysis for the total yield and average secondary gamma-ray energy as a function of the incident neutron energy are presented in Figs. 21-24. Two values of the lower cut-off in gamma-ray energy were used, 0.22 and $0.62 \mathrm{MeV}$. Comparisons are made for the same quantities calculated from the evaluated files.

The data shown in the graphs are given in the tables contained in the last section of the report. The values shown in the graphs and presented in the tables do not include an uncertainty of $10 \%$ in overall normalization due mainly to the determination of the incident neutron flux.

\section{ACKNOWLEDGMENTS}

We wish to thank J. W. McConnell for help with the electronics. J. G. Craven for aid with the computers and H. A. Todd and the ORELA staff for operation of the accelerator. We appreciate the help of R. E. McDonald in obtaining the manganese sample. W. E. Ford III helped greatiy in preparing cross sections from the evaluated data file. 
1. G. L. Morgan, T. A. Love, J. K. Dickens and F. G. Perey, "Gamma-Ray Production Cross Sections of Tantalum and Carbon for Incident Neutron Energies Between 0.007 and 20.0 MeV," ORNL-TM-3702 (February 1972).

J. K. Dickens, G. L. Morgan and F. G. Perey, "Gamma-Ray Production Due to Interactions with Iron for Incident Neutron Energies Between 0.8 and $20 \mathrm{MeV}$ : Tabulated Differential Cross Sections," ORNL-4798 (August 1972).

J. K. Dickens, T. A. Love and G. L. Morgan, "Gamma-Ray Production Due to Neutron Interactions with Tungsten for Incident Neutron Energies Between 1.0 and $20 \mathrm{MeV}$ : ' Tabulated Differential Cross Sections," ORNL-4847 (January 1973).

J. K. Dickens, T. A. Love and G. L. Morgan, "Gamma-Ray Production Due to Neutron Interactions with Copper for Incident Neutron Energies Between 2.0 and $20 \mathrm{MeV}$ : Tabulated Differential Cross Sections," ORNL-4846 (January 1973).

J. K. Dickens, T. A. Love and G. L. Morgan, "Gamma-Ray Production Due to Neutron Interactions with Nitrogen for Incident Neutron Energies Between 2.0 and $20 \mathrm{MeV}$ : Tabulated Differential Cross Sections," ORNL-4864 (April 1973).

J. K. Dickens, T. A. Love and G. L. Morgan, "Gamma-Ray Production Due to Neutron Interactions with Aluminum for Incident Neutron Energies Between 0.85 and $20 \mathrm{MeV}$ : Tabulated Differential Cross Sections," ORNL-TM-4232 (July 1973).

J. K. Dickens, T. A. Love and G. L. Morgan, "Gamma-Ray Production Due to Neutron Interactions with Calcium for Incident Neutron Energies Between 0.85 and $20 \mathrm{MeV}$ : Tabulated Differential Cross Sections," ORNL-TM-4252 (July 1973).

J. K. Dickens, T. A. Love and G. L. Morgan, "Gamma-Ray Production Due to Neutron Interactions with Nickel for Incident Neutron Energies Between 1.0 and $20 \mathrm{MeV}$ : Tabulated Differential Cross Sections," ORNL-TM-4379 (November 1973).

J. K. Dickens, T. A. Love and G. L. Morgan, "Gamma-Ray Production from Neutron Interactions with Silicon for Incident Neutron Energies Between 1.0 and $20 \mathrm{MeV}$ : Tabulated Differential Cross Sections," ORNL-TM-4389 (December 1973).

J. K. Dickens, T. A. Love and G. L. Morgan, "Gamma-Ray Production Due to Neutron Interactions with Tin for Incident Neutron Energies Between 0.75 and $20 \mathrm{MeV}$ : Tabulated Differential Cross Sections," ORNL-TM-4406 (November 1973). 
J. K. Dickens, T. A. Love and G. L. Morgan, "Gamma-Ray Production Due to Neutron Interactions with Zinc for Incident Neutron Energies Between 0.85 and $20 \mathrm{MeV}$ : Tabulated Differential Cross Sections," ORNL-TM-4464 (February 1974).

J. K. Dickens, T. A. Love and G. L. Morgan, "Gamma-Ray Production Due to Neutron Interactions with Fluorine and Lithium for Incident Neutron Energies Between 0.85 and $20 \mathrm{MeV}$ : Tabulated Differential Cross Sections," ORNL-TM-4538 (April 1974).

J. K. Dickens, T. A. Love and G. L. Morgan, "Gamma-Ray Production Due to Neutron Interactions with Magnesium for Incident Neutron Energies Between 0.8 and $20 \mathrm{MeV}$ : Tabulated Differential Cross Sections," ORNL-TM-4544 (May 1974).

G. T. Chapman and G. L. Morgan, "The $\mathrm{Pb}(n, x y)$ Reaction for Incident Neutron Energies Between 0.6 and $20.0 \mathrm{MeV}, "$ ORNL-TM-4822 (February 1975).

G. L. Morgan and J. K. Dickens, "Production of Low Energy Gamma Rays by Neutron Interactions with Fluorine for Incident Neutron Energies Between 0.1 and $20 \mathrm{MeV}, "$ ORNL-TM-4823 (February 1975).

G. L. Morgan and E. Newman, "The $A u(n, x y)$ Reaction Cross Section for Incident Neutron Energies Between 0.2 and 20.0 MeV," ORNL-TM-4973 (August 1975).

J. K. Dickens, G. L. Morgan and E. Newman, "The $\mathrm{Nb}(n, \mathrm{x} \gamma)$ Reaction Cross Section for Incident Neutron Energies Between 0.65 and 20.0 MeV," ORNL-TM-4972 (September 1975).

J. K. Dickens, T. A. Love and G. L. Morgan, "Gamma-Ray Production Due to Neutron Interactions with Silver for Incident Neutron Energies Between 0.3 and $20 \mathrm{MeV}$ : Tabulated Differential Cross Sections," ORNL-TM-5081 (October 1975).

G. L. Morgan and E. Newman, "The $\mathrm{Cr}(n, x y)$ Reaction Cross Section for Incident Neutron Energies Between 0.2 and 20.0 MeV," ORNL-TM-5098, ENDF-222 (November 1975).

G. L. Morgan and E. Newman, "The Mo $(n, x y)$ Reaction Cross Section for Incident Neutron Energies Between 0.2 and $20.0 \mathrm{MeV}$," ORNL-TM-5097, ENDF-220 (December 1.975).

G. T. Chapman, "The $\mathrm{Cu}(n, \mathrm{x} \gamma)$ Reaction Cross Section for Incident Neutron Energies Between 0.2 and $20.0 \mathrm{MeV}, "$ ORNL/TM-5215 (February 1976).

E. Newman and G. L. Morgan, "The $V(n, x y)$ Reaction Cross Section for Incident Neutron Energies Between 0.2 and $20.0 \mathrm{MeV}, "$ ORNL/TM-5299 (April 1976). 
2. H. Takahashi, ENDF/B MAT 1197, Brookhaven National Laboratory (November 1974).

3. J. K. Dickens, G. L. Morgan and F. G. Perey, Nucl. Sci. Eng. 50, 311 (1973).

4. Frances Pleasonton, Robert L. Ferguson and H. W. Schmitt, Phys. Rev. C $\underline{6}, 1023$ (1972).

5. G. L. Morgan, T. A. Love and F. G. Perey, "Integral Neutron Scattering Measurements on Carbon from 1 to $20 \mathrm{MeV}$," ORNL-TM-4157 (April 1973). 


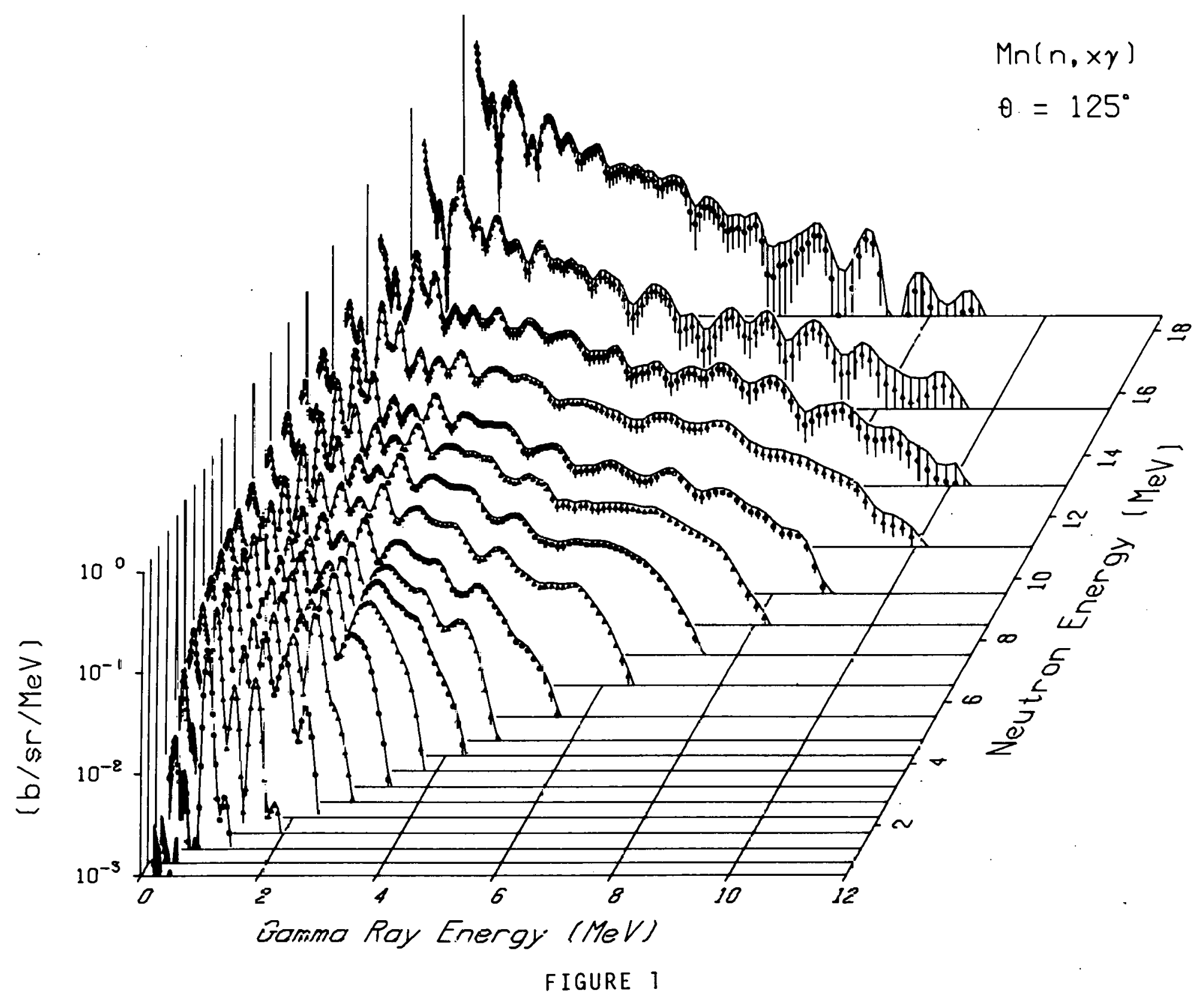




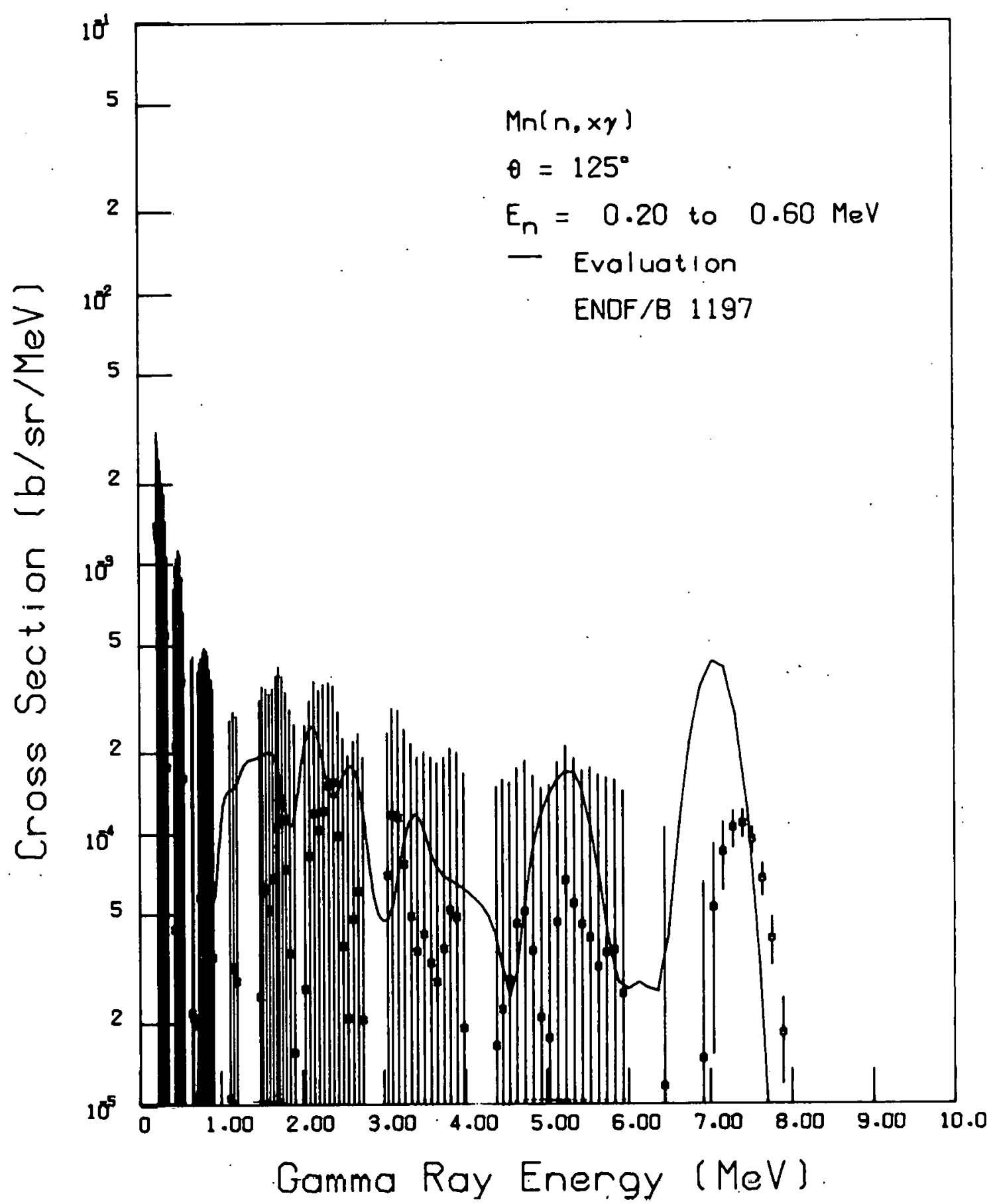

FIGURE 2 


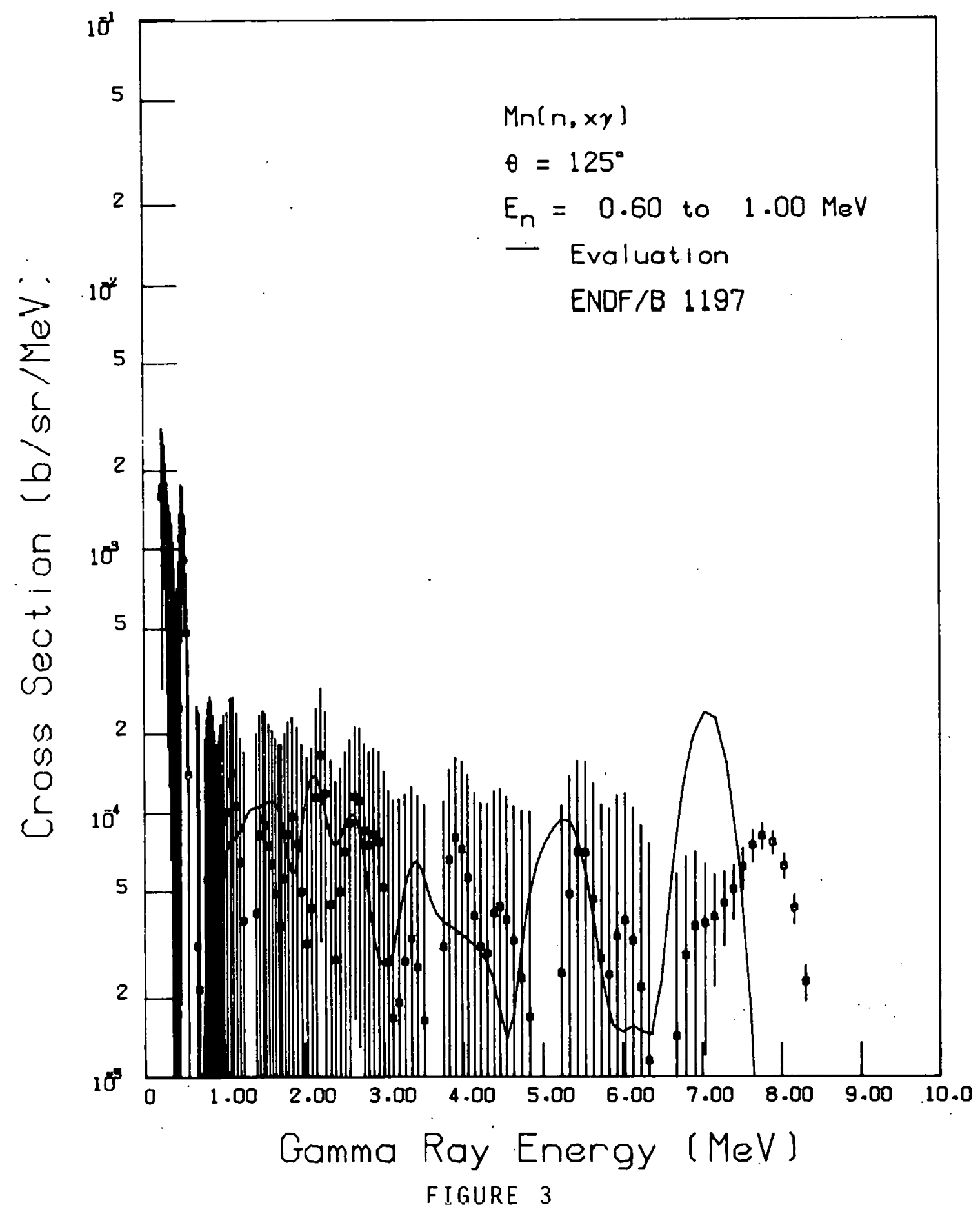




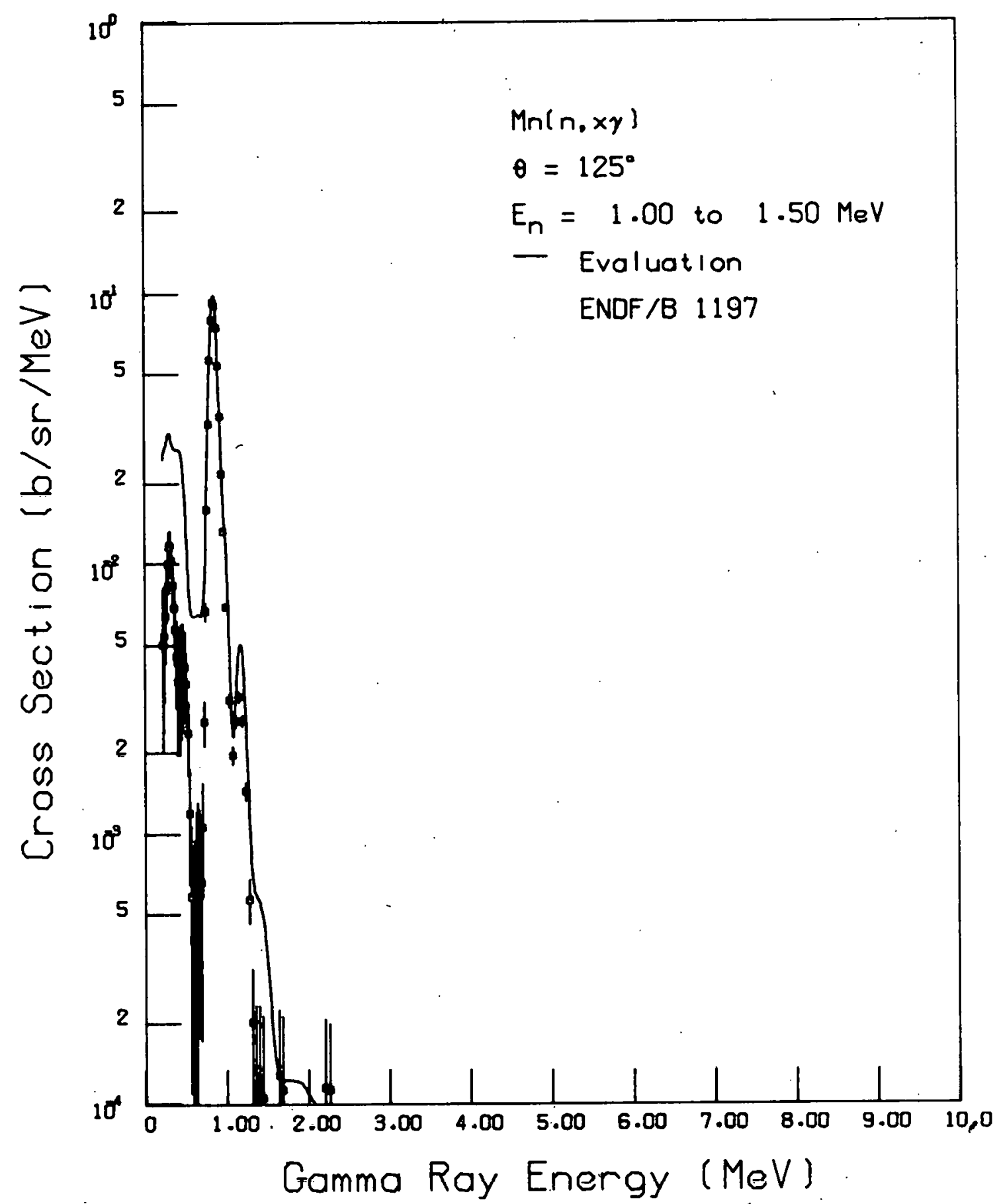

FIGURE 4 


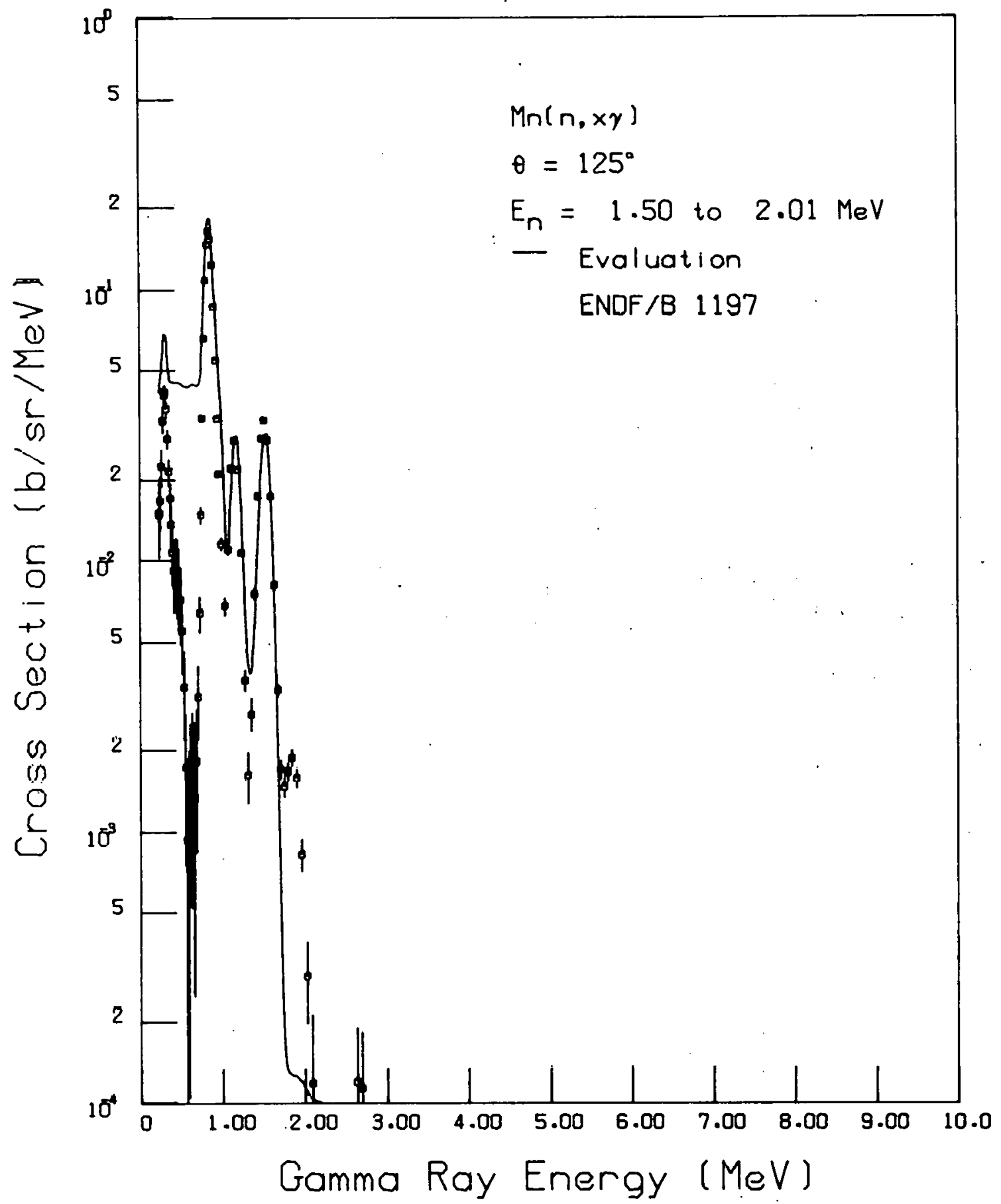

FIGURE 5 


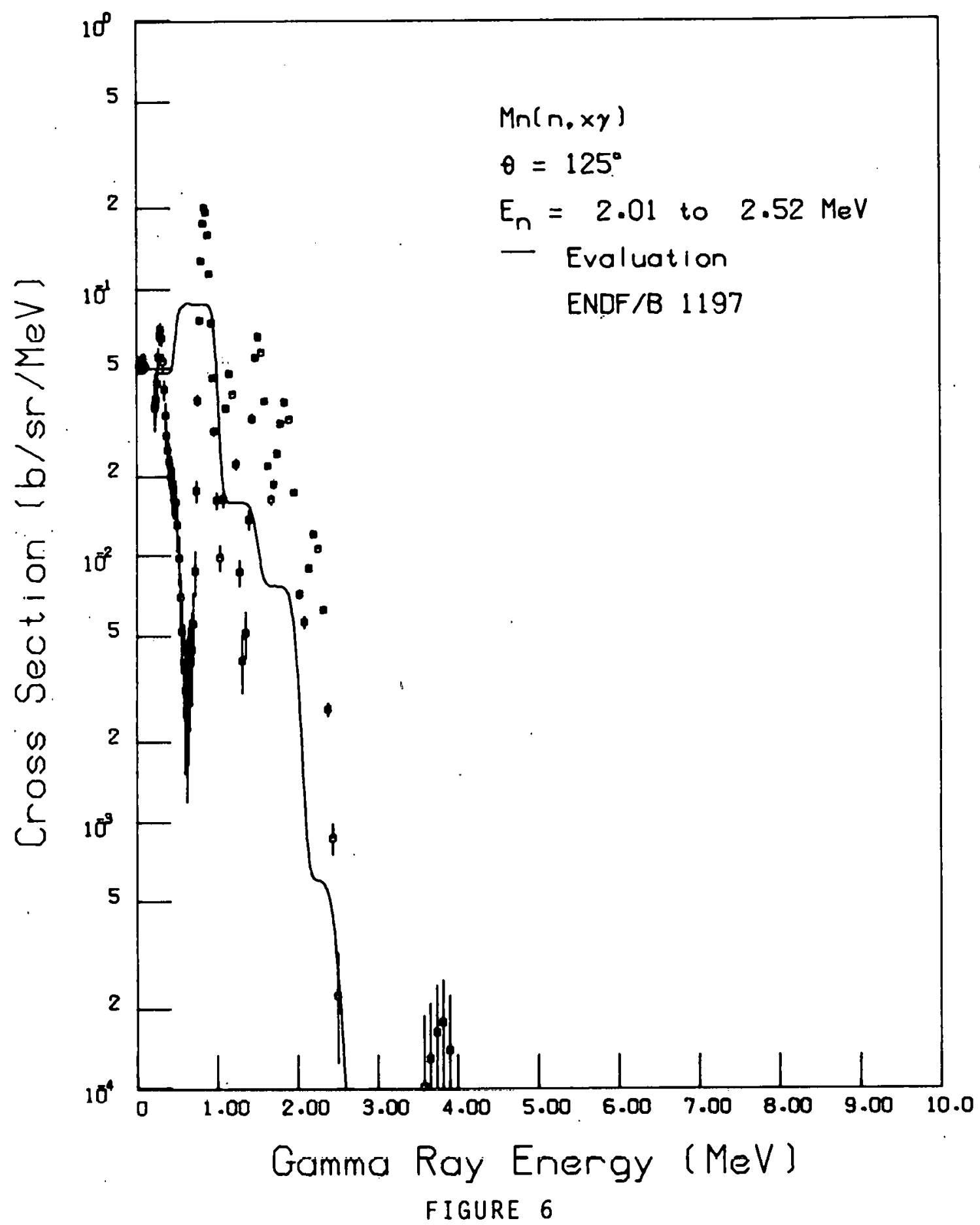




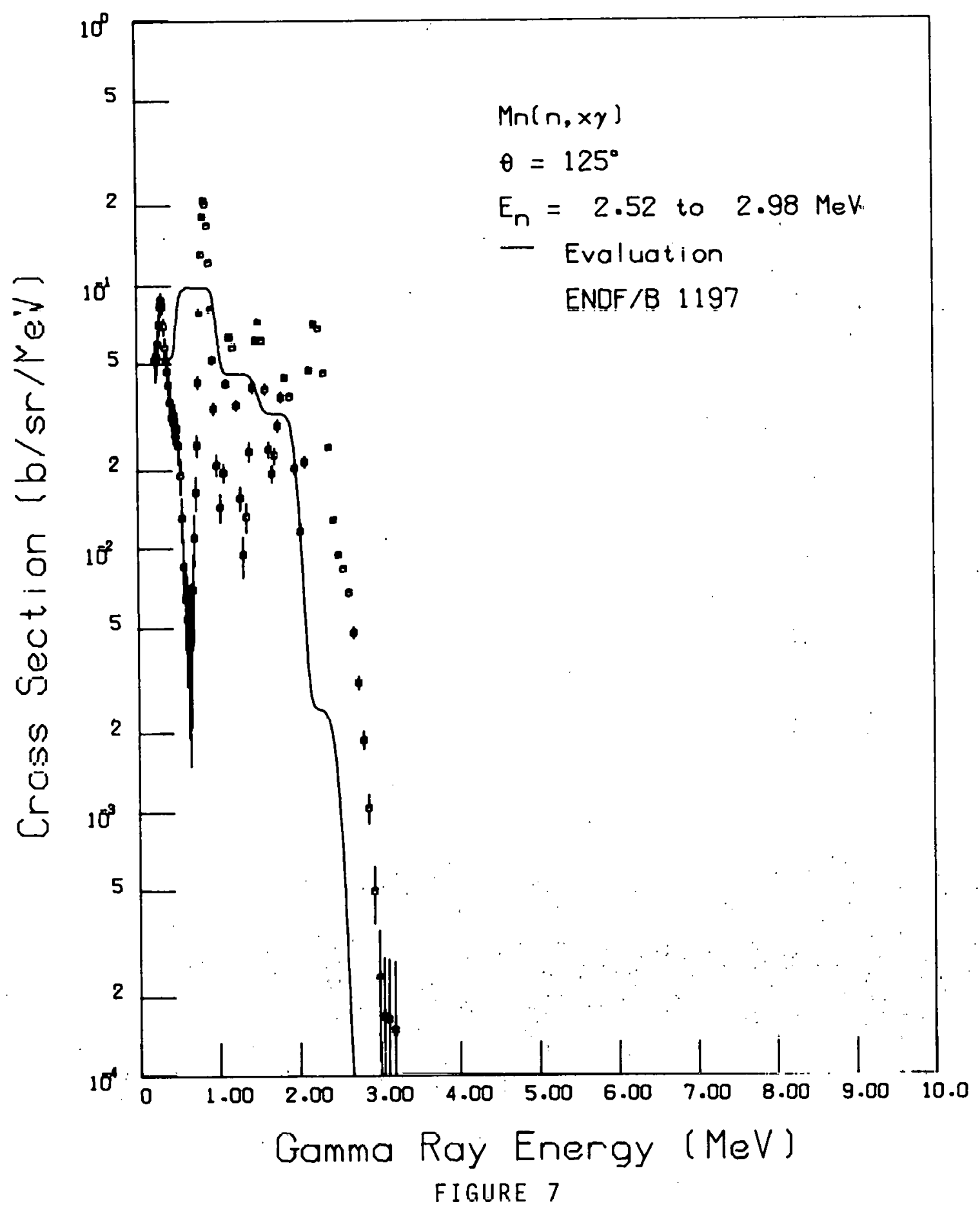




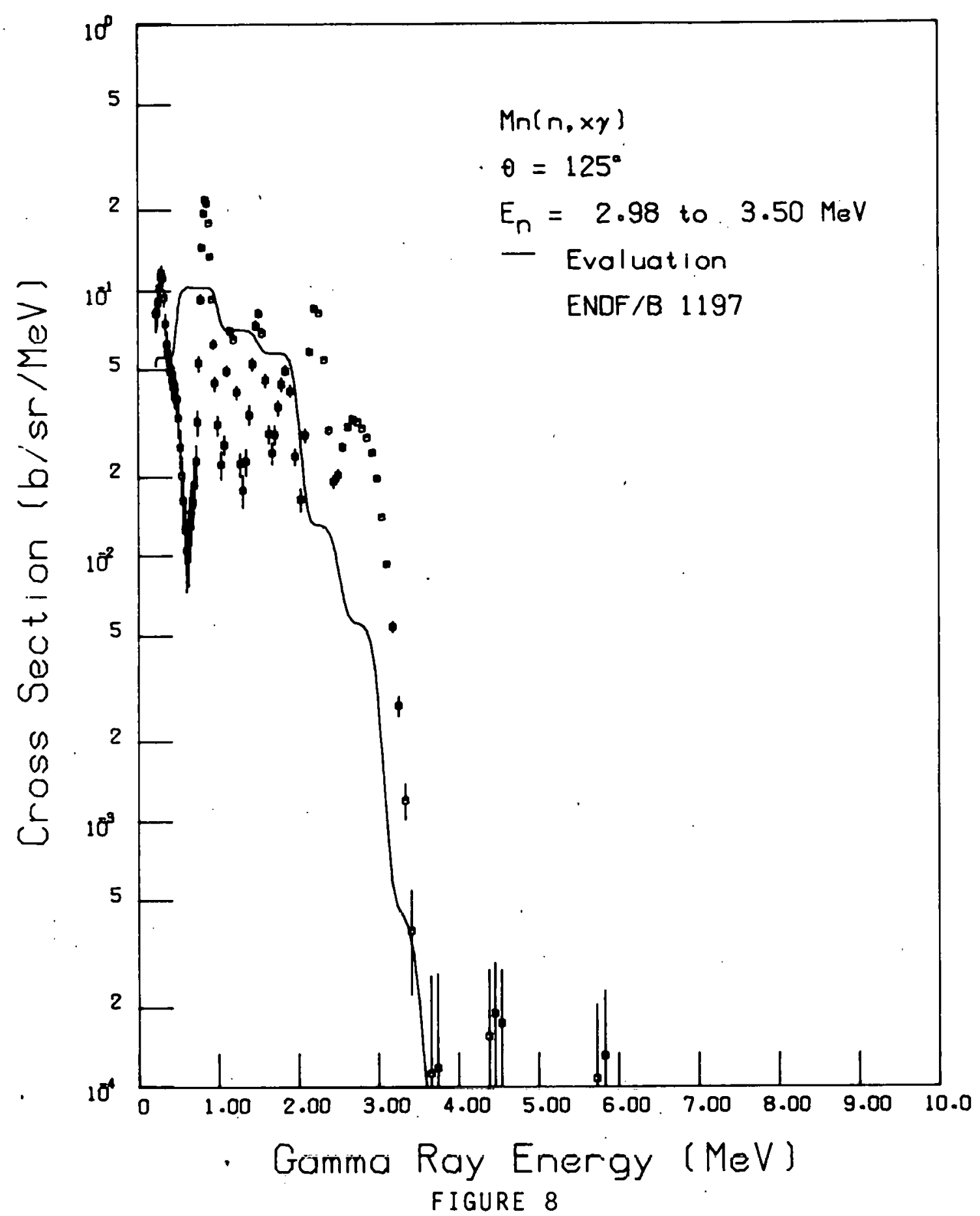




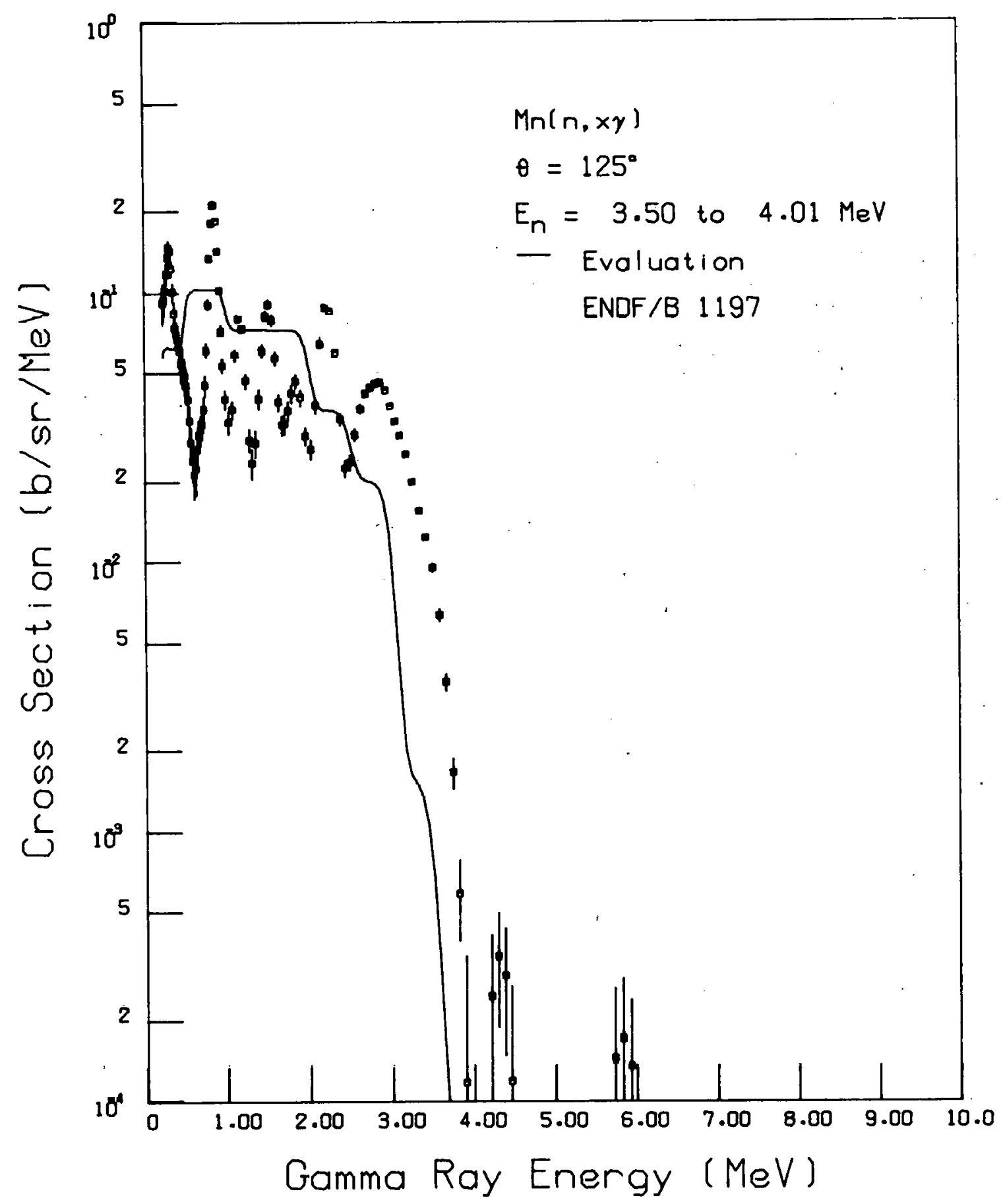

FIGURE 9 


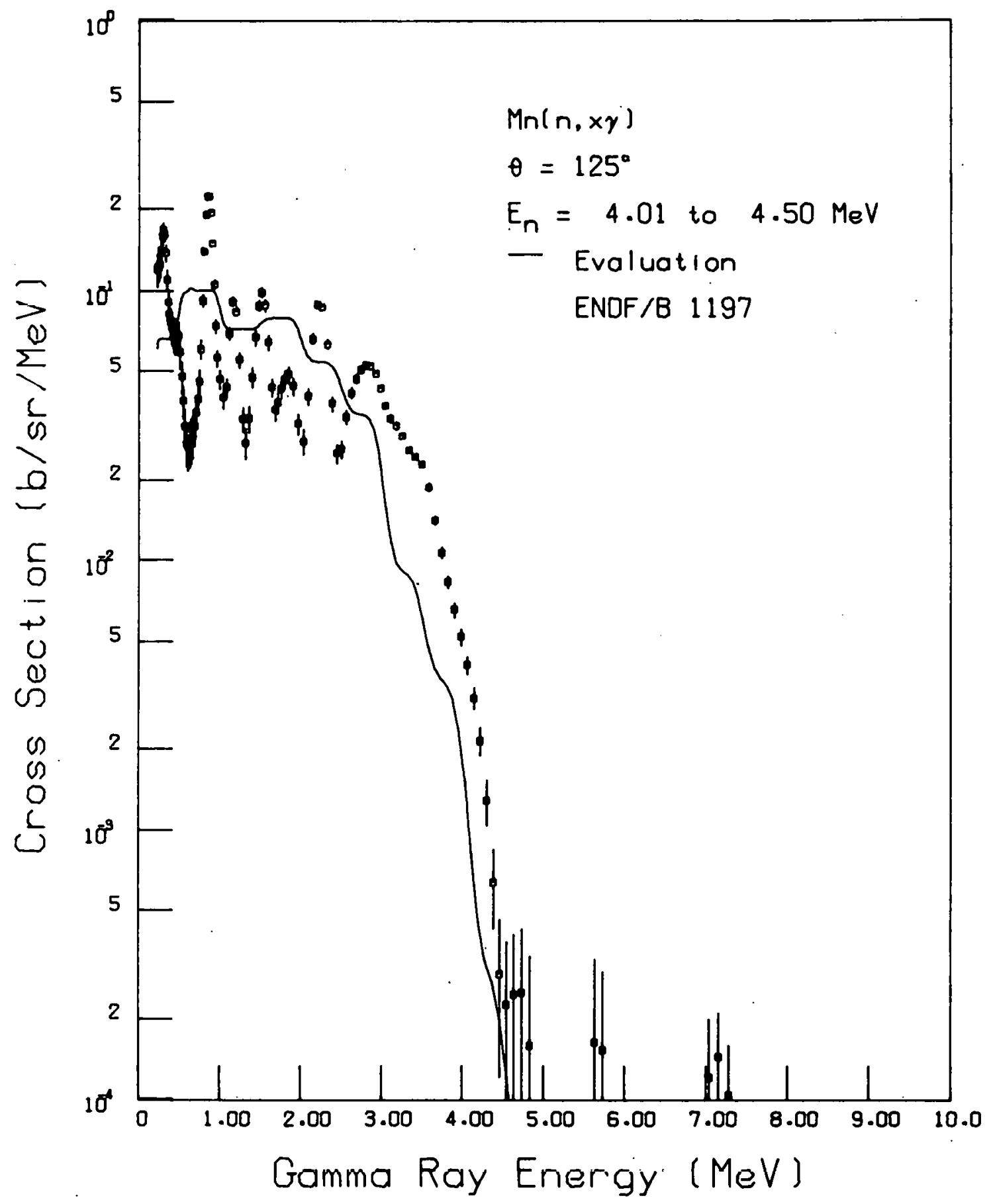

FIGURE 10 


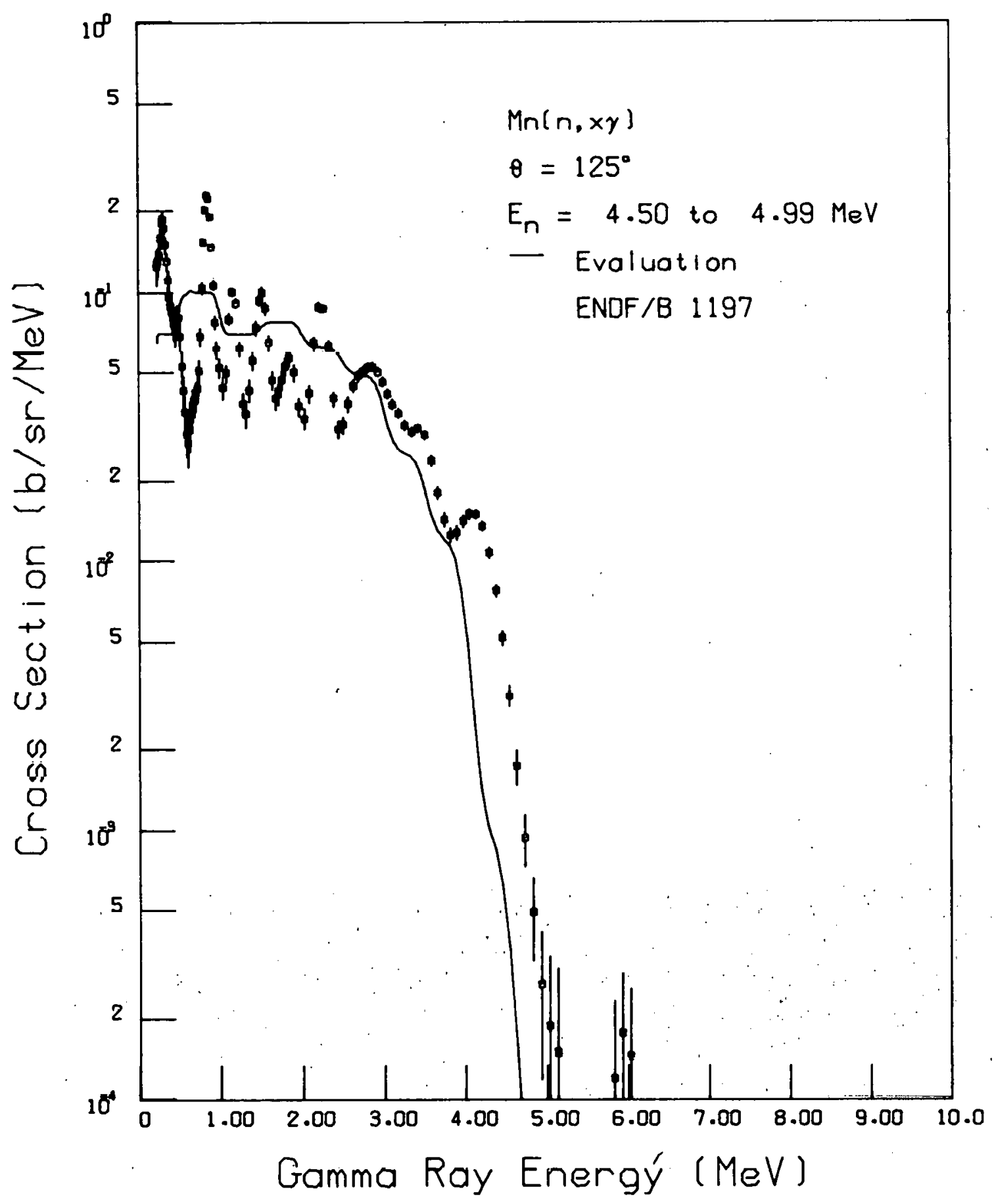

FIGURE 11 


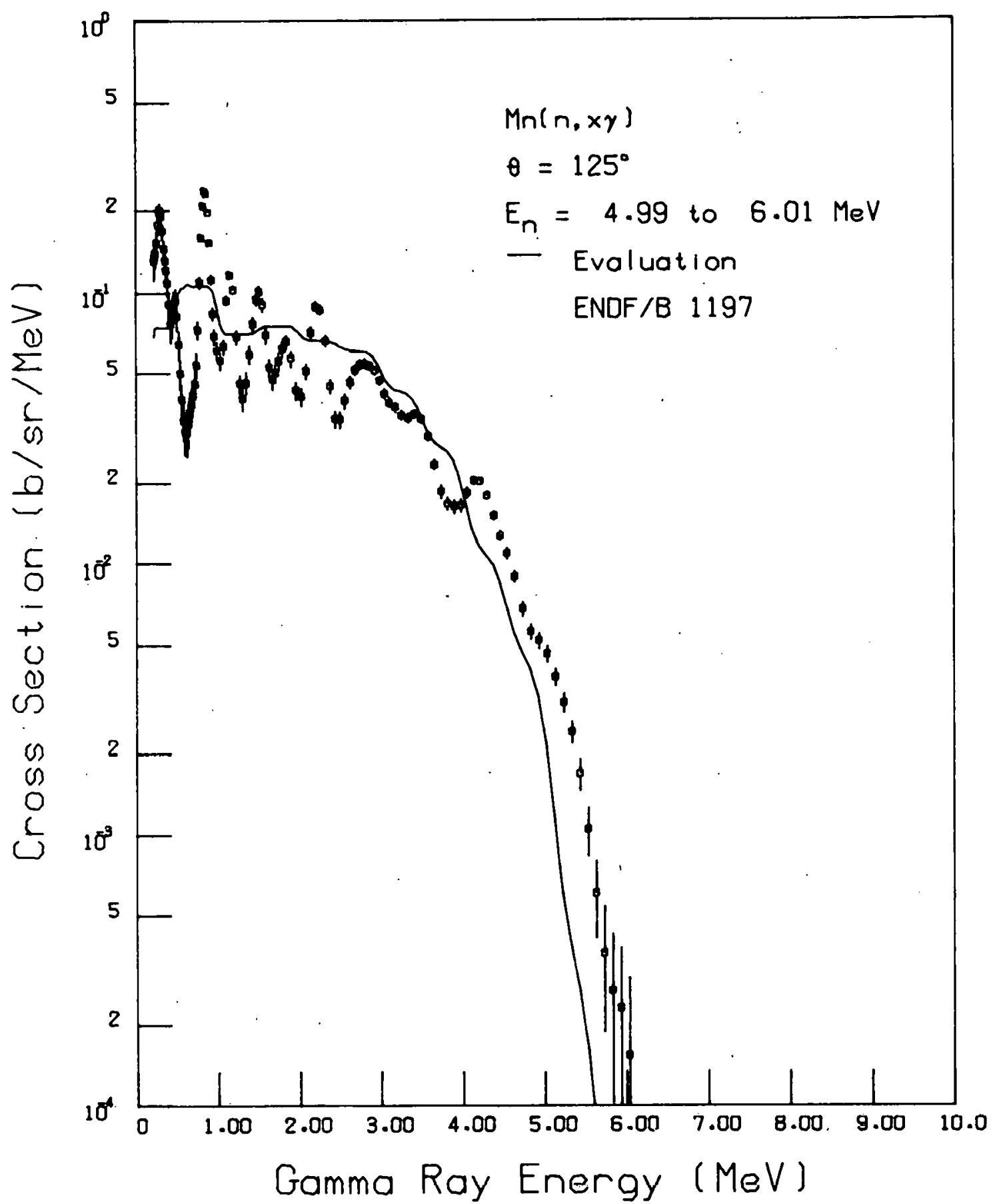

FIGURE 12 


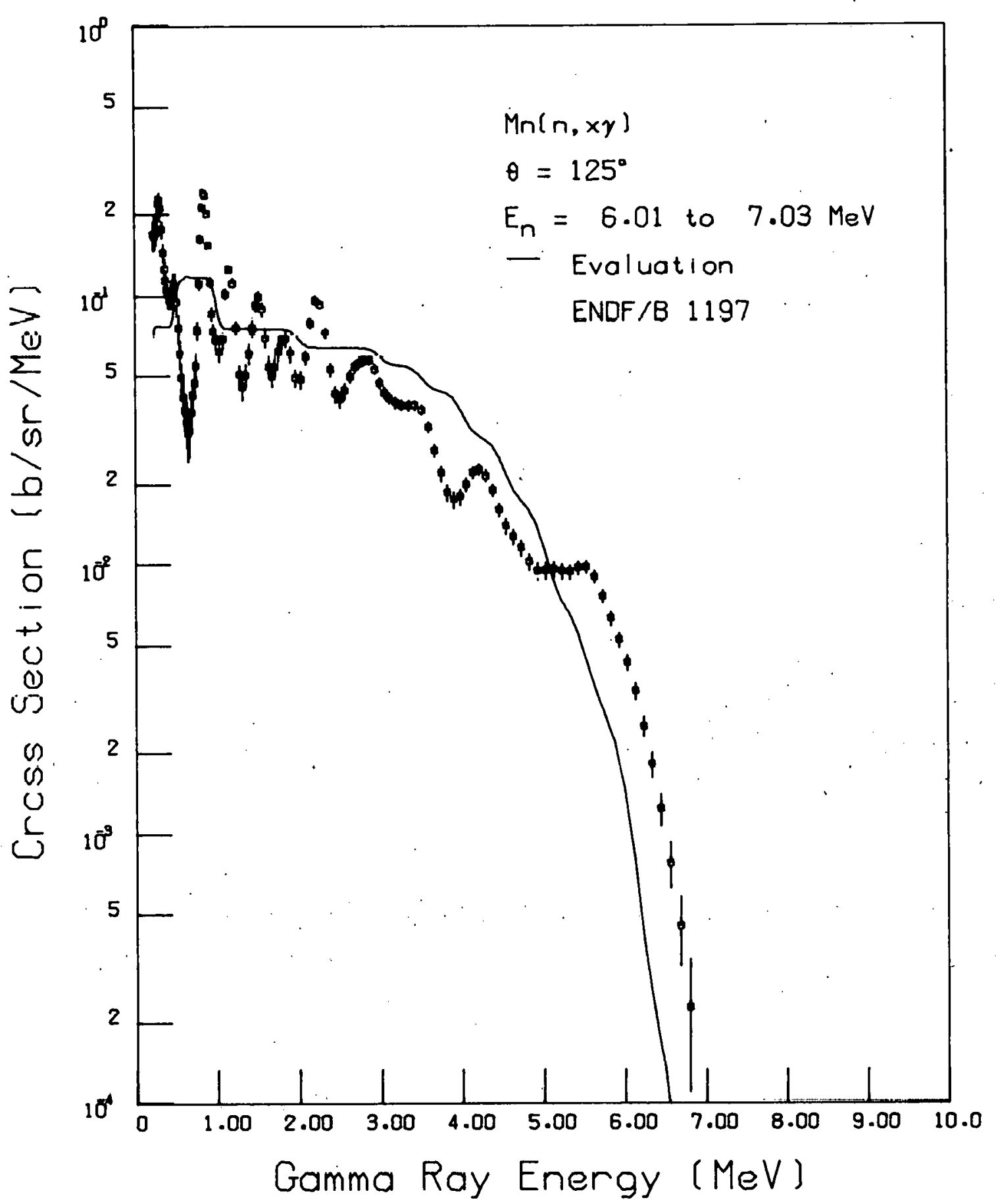

FIGURE 13 


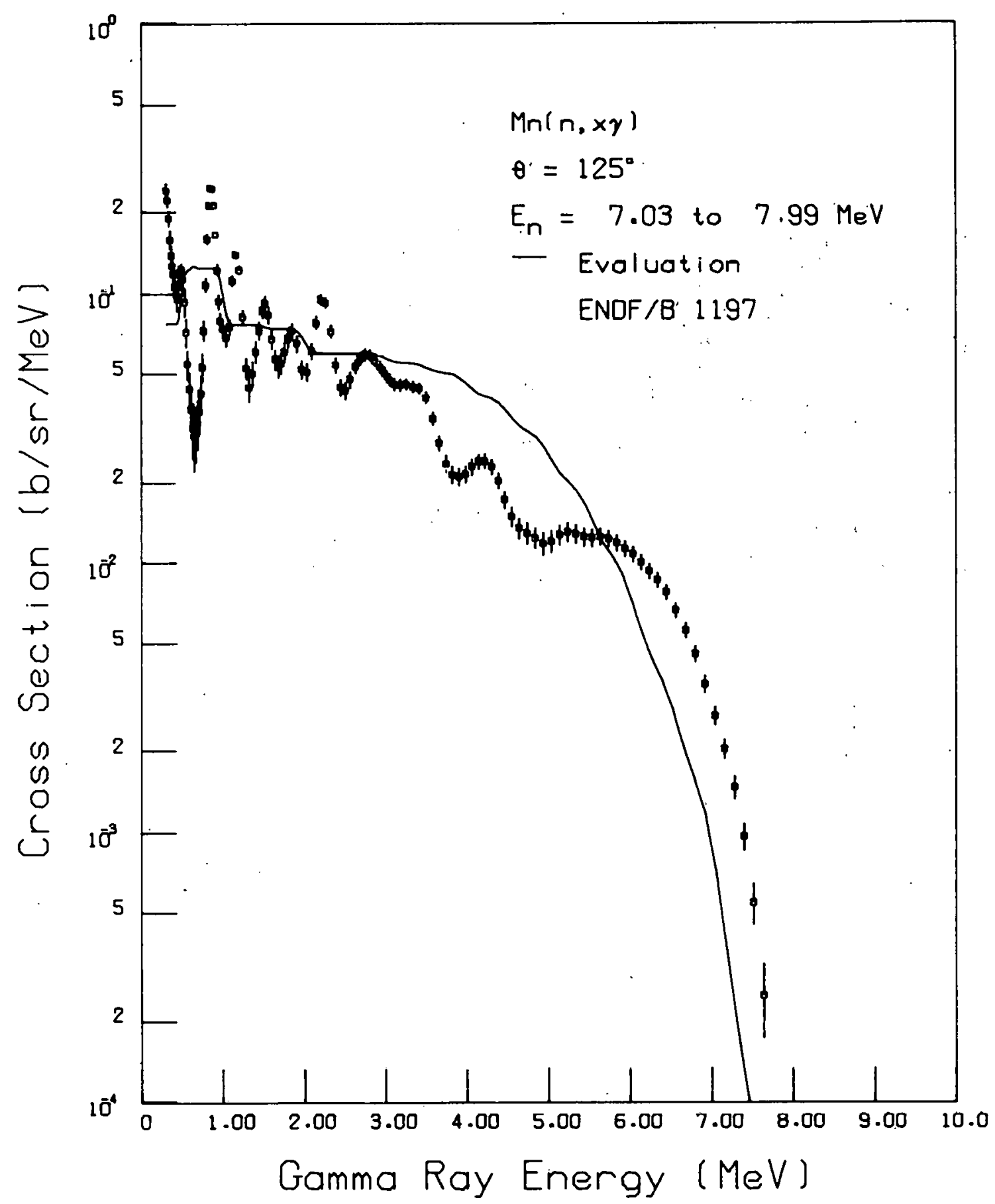

FIGURE 14 


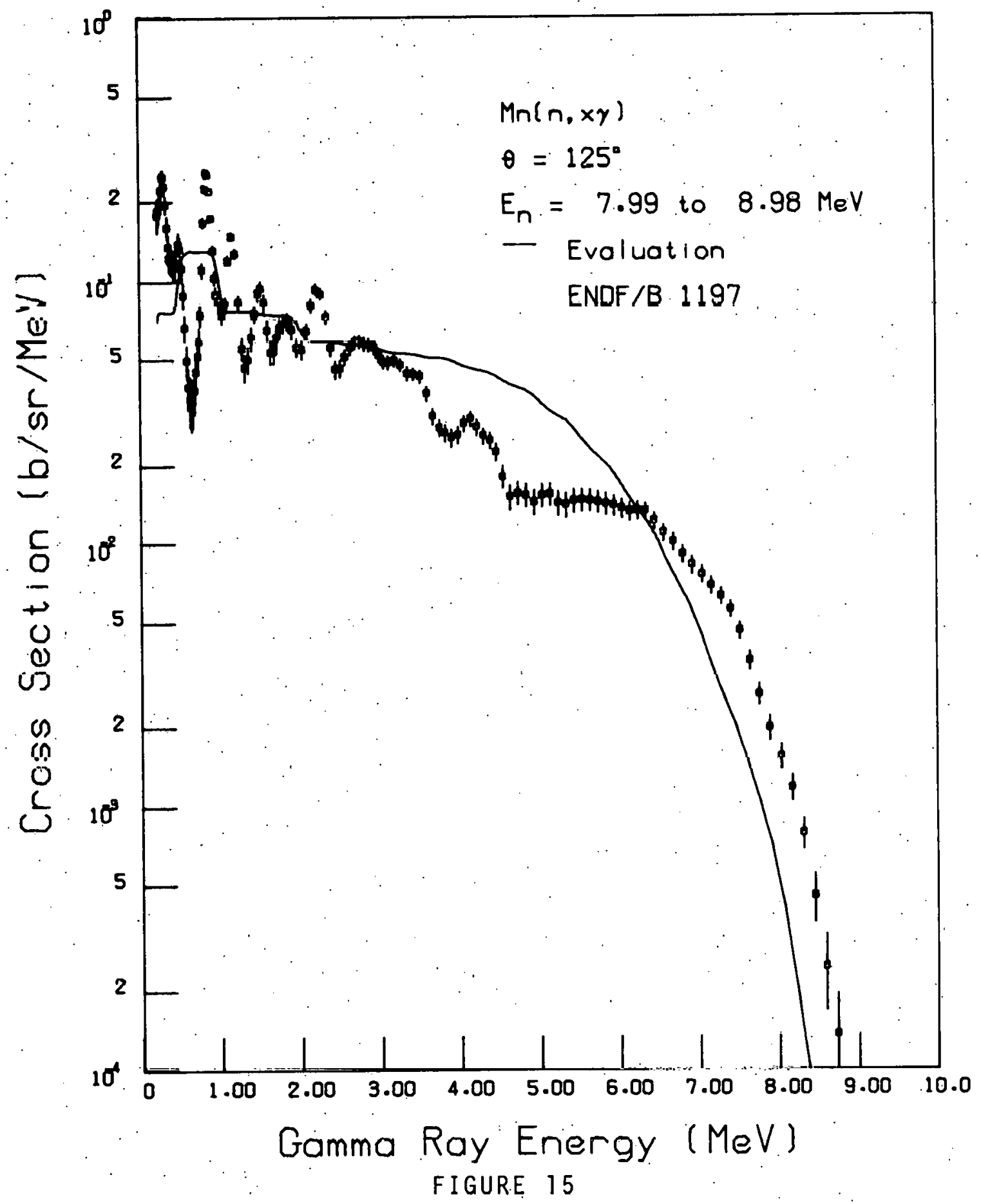




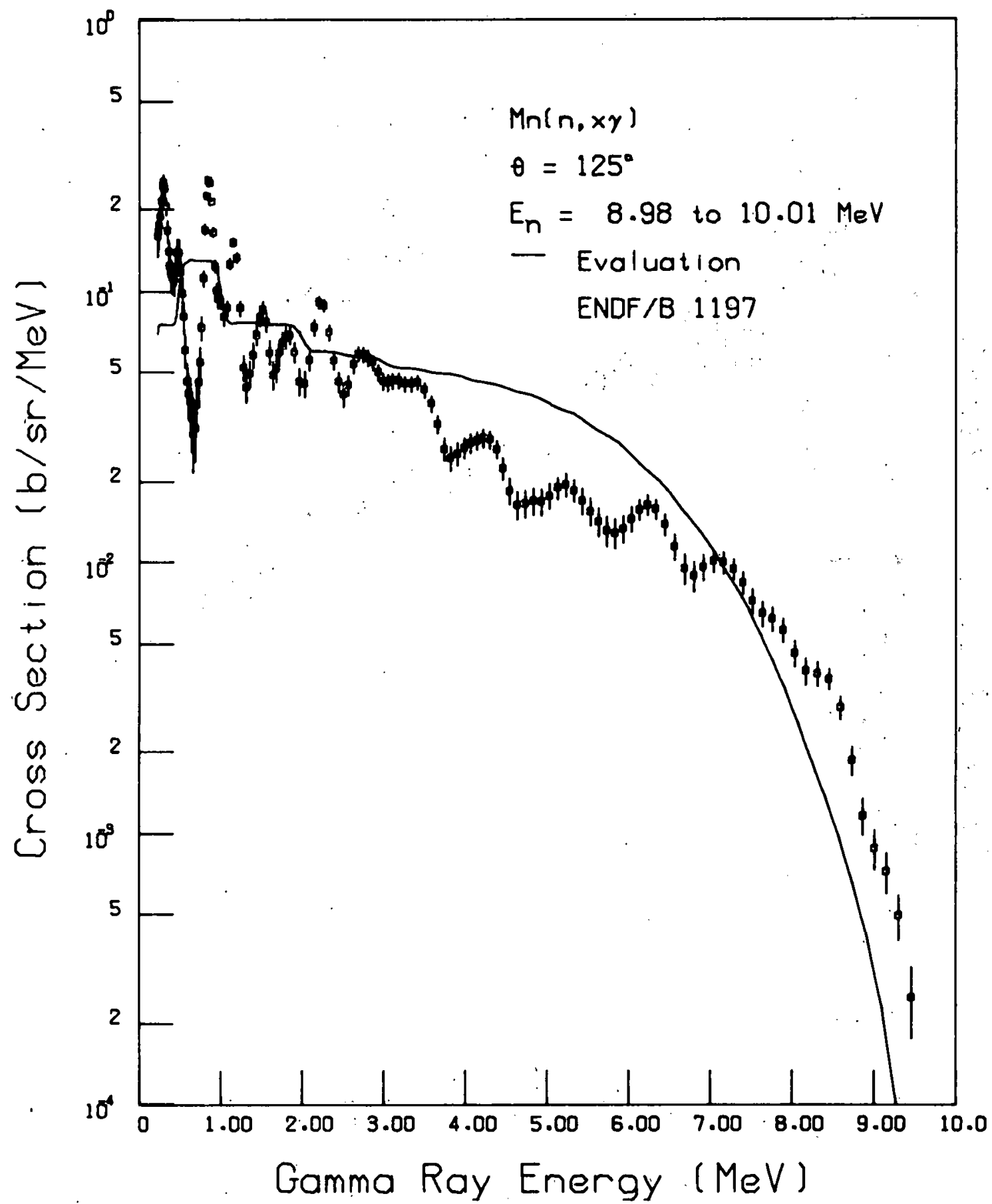

FIGURE I6 


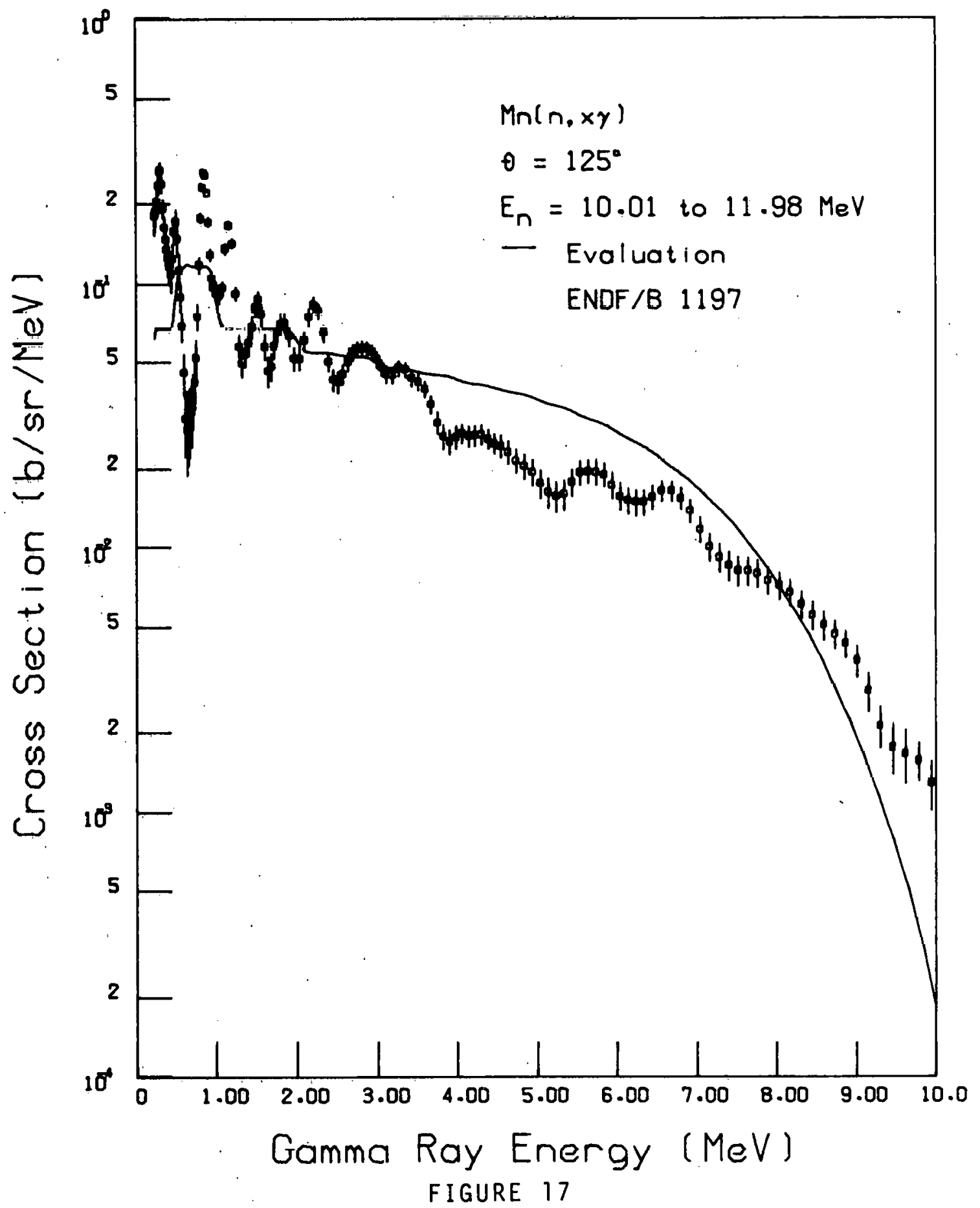




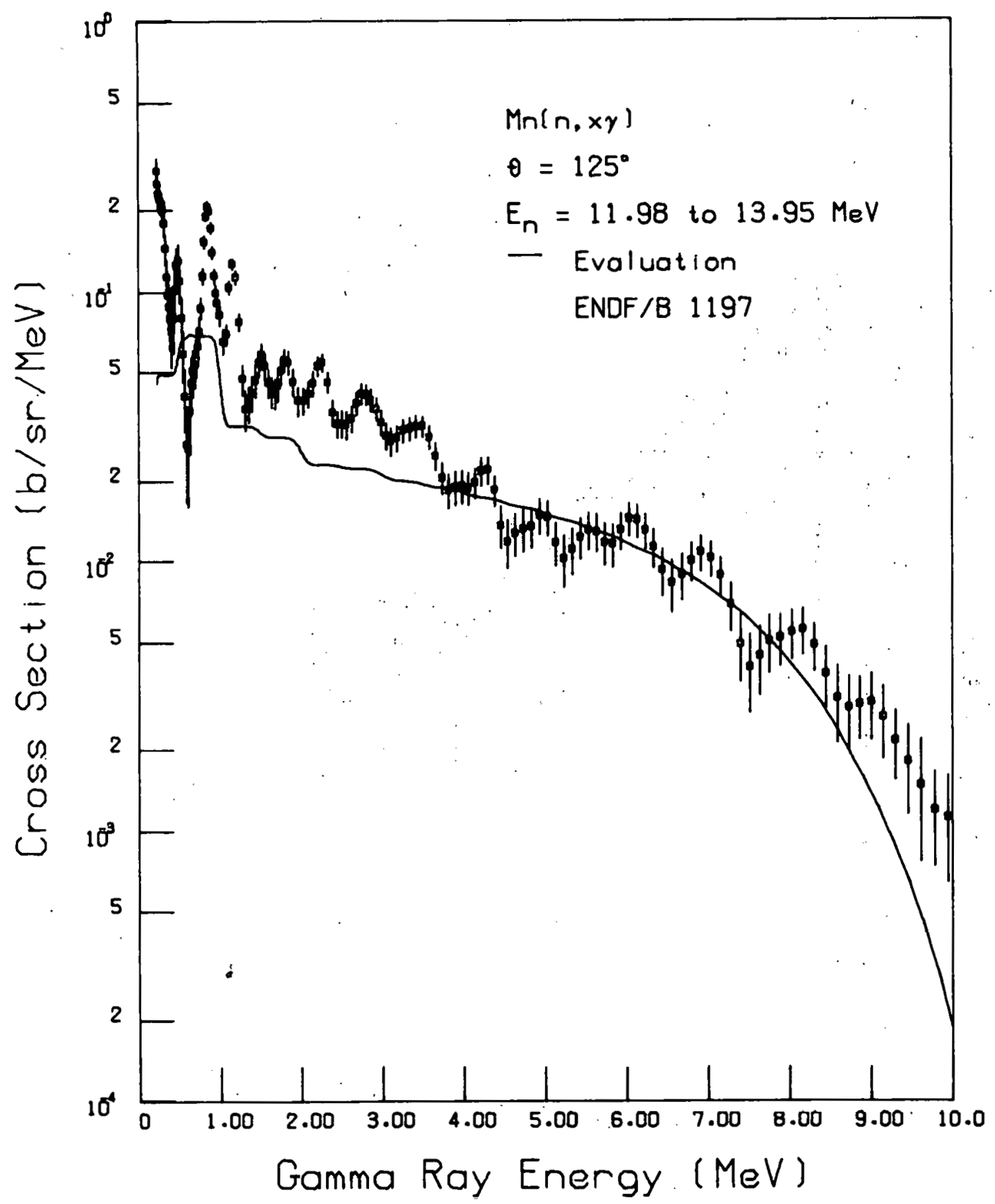

FIGURE 18 


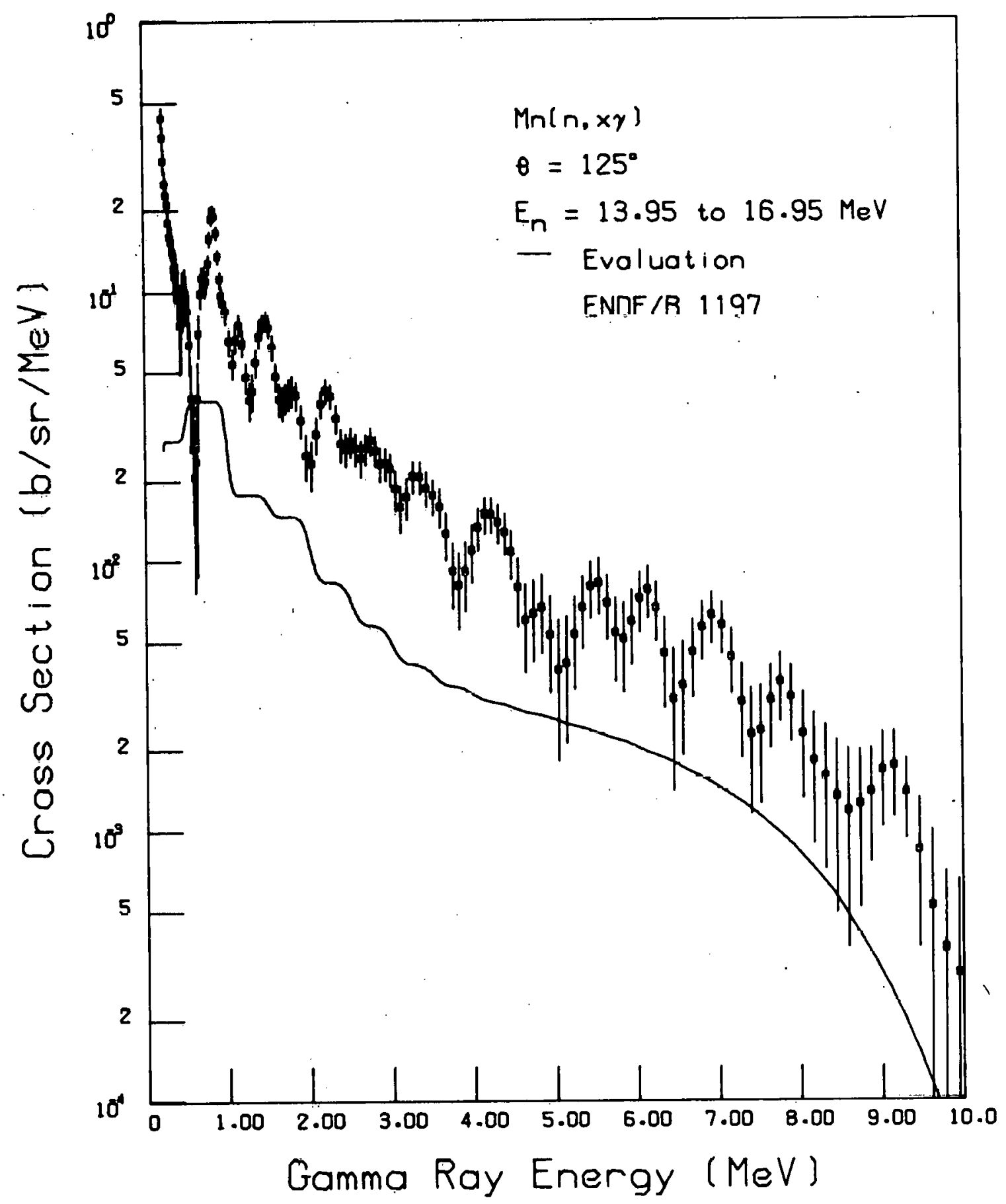

FIGURE 19 


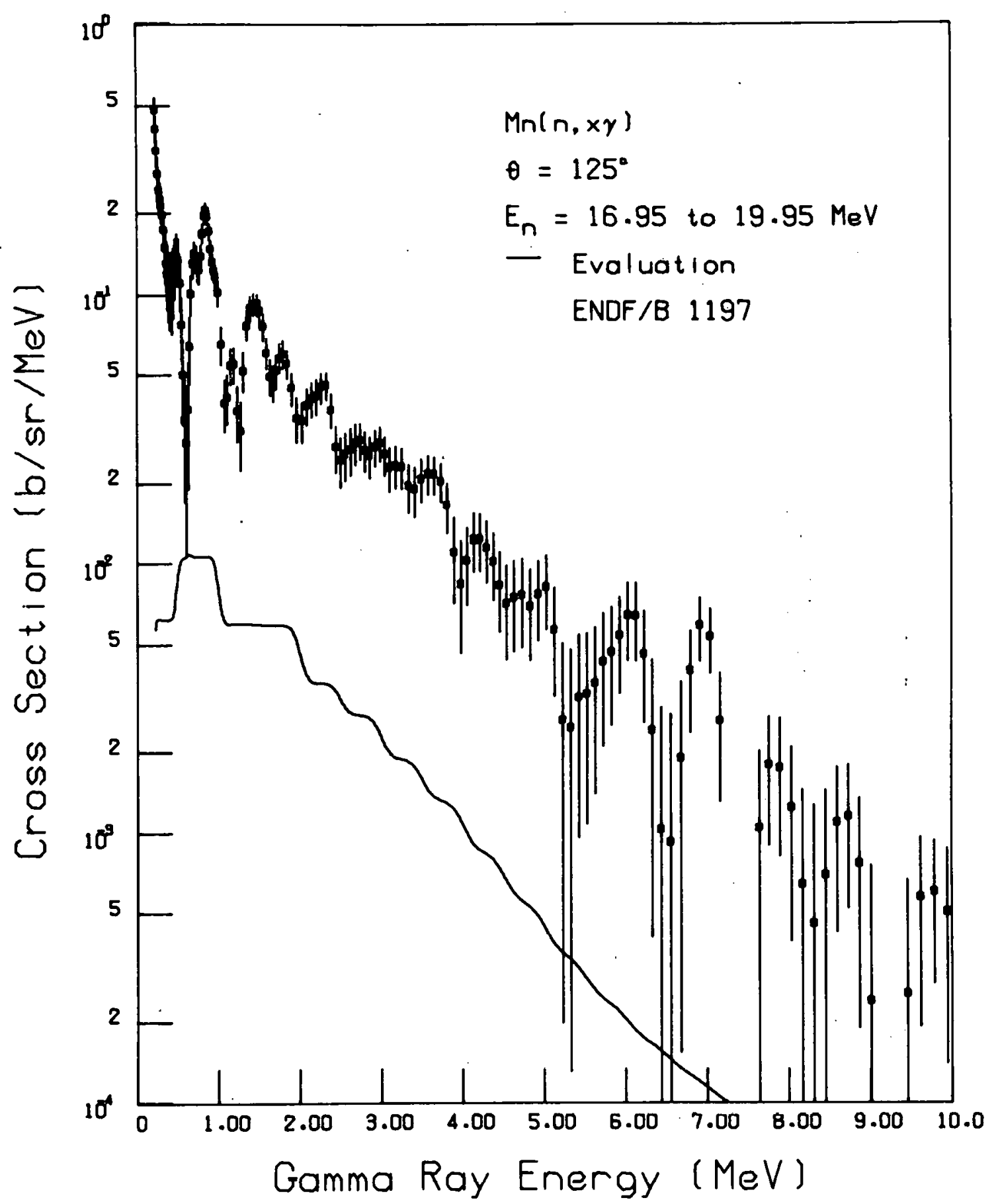

FIGURE 20 


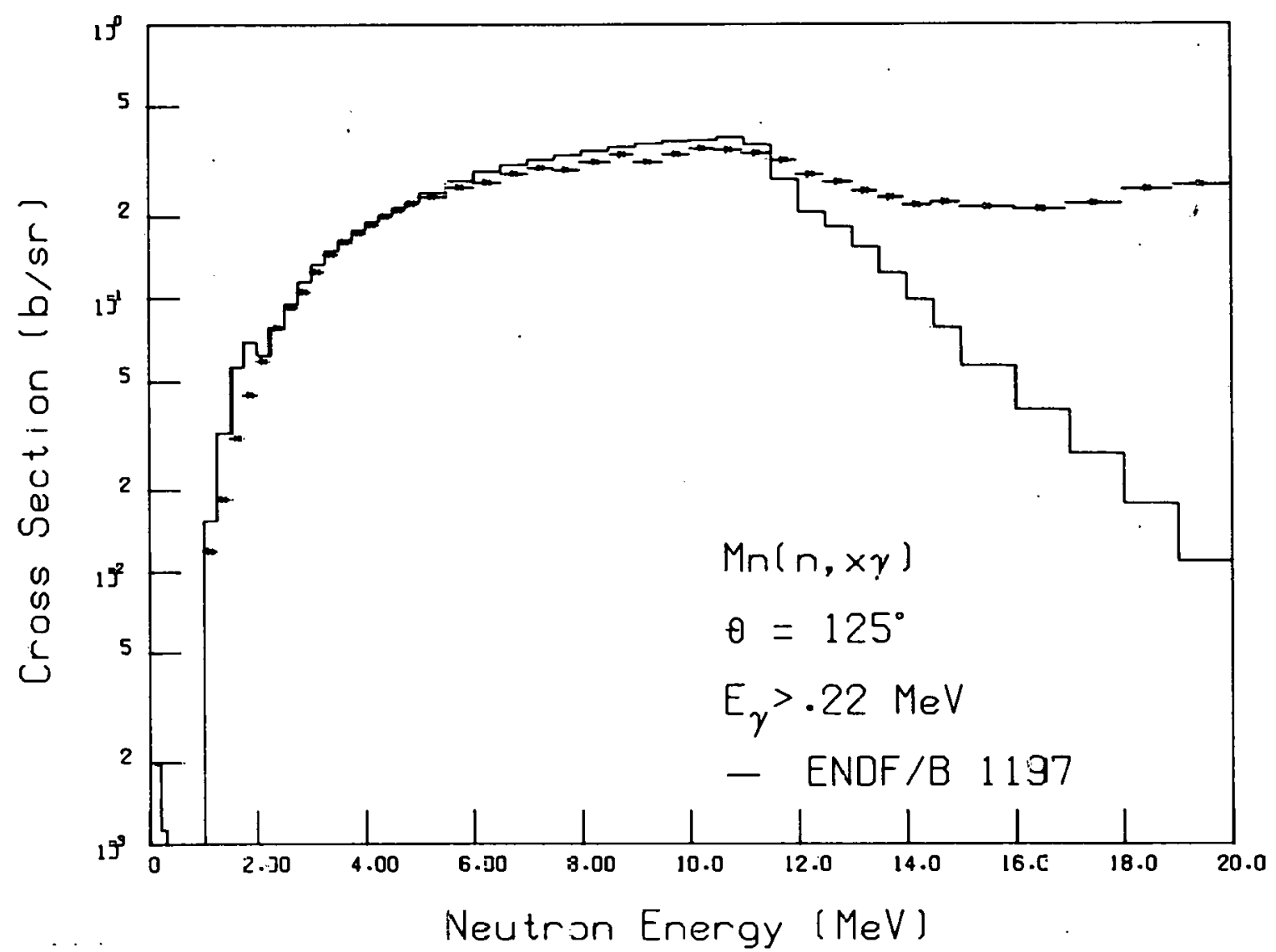

$\stackrel{\infty}{\infty}$

FIGURE 21 


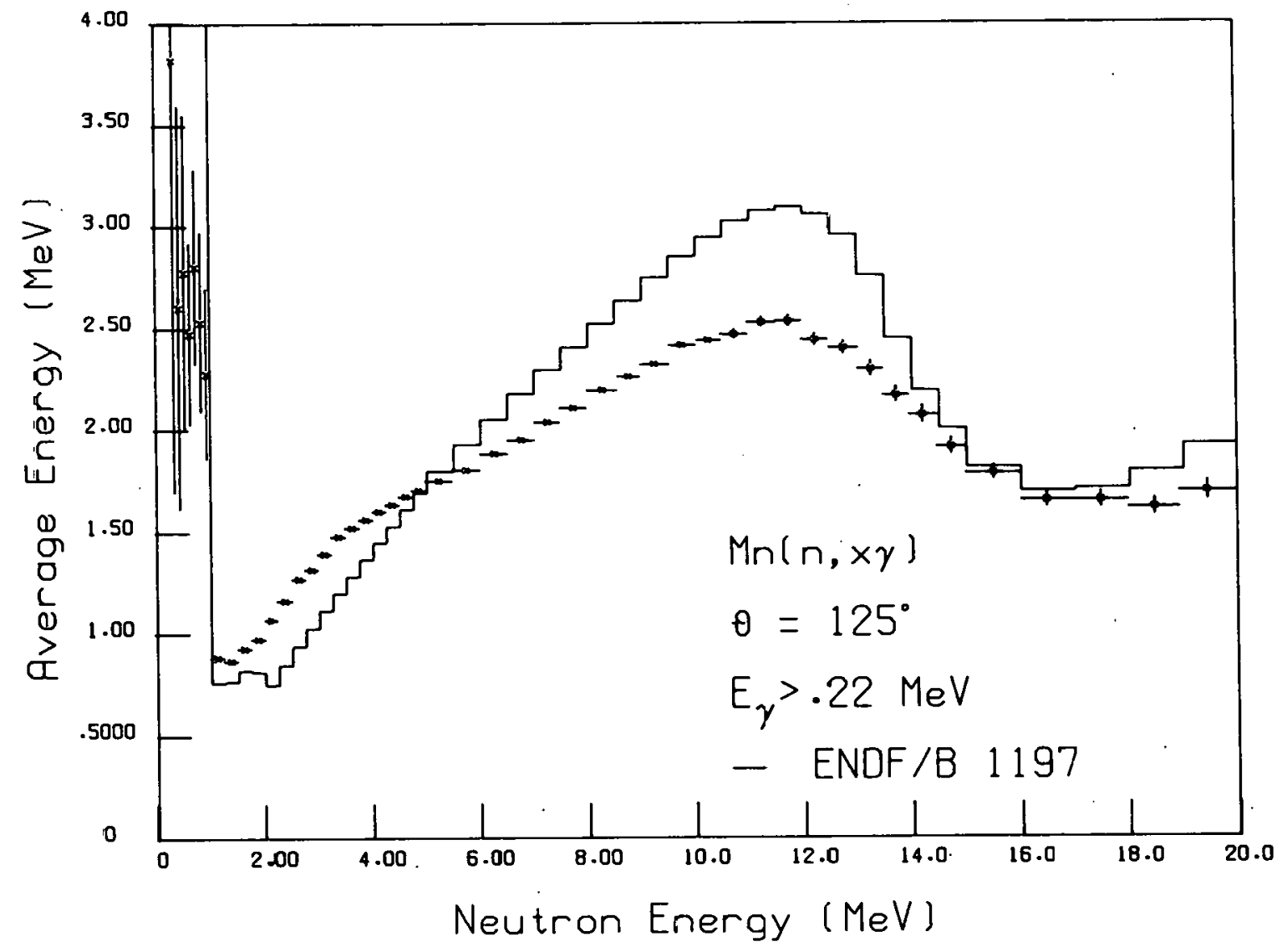

FIGURE 22 


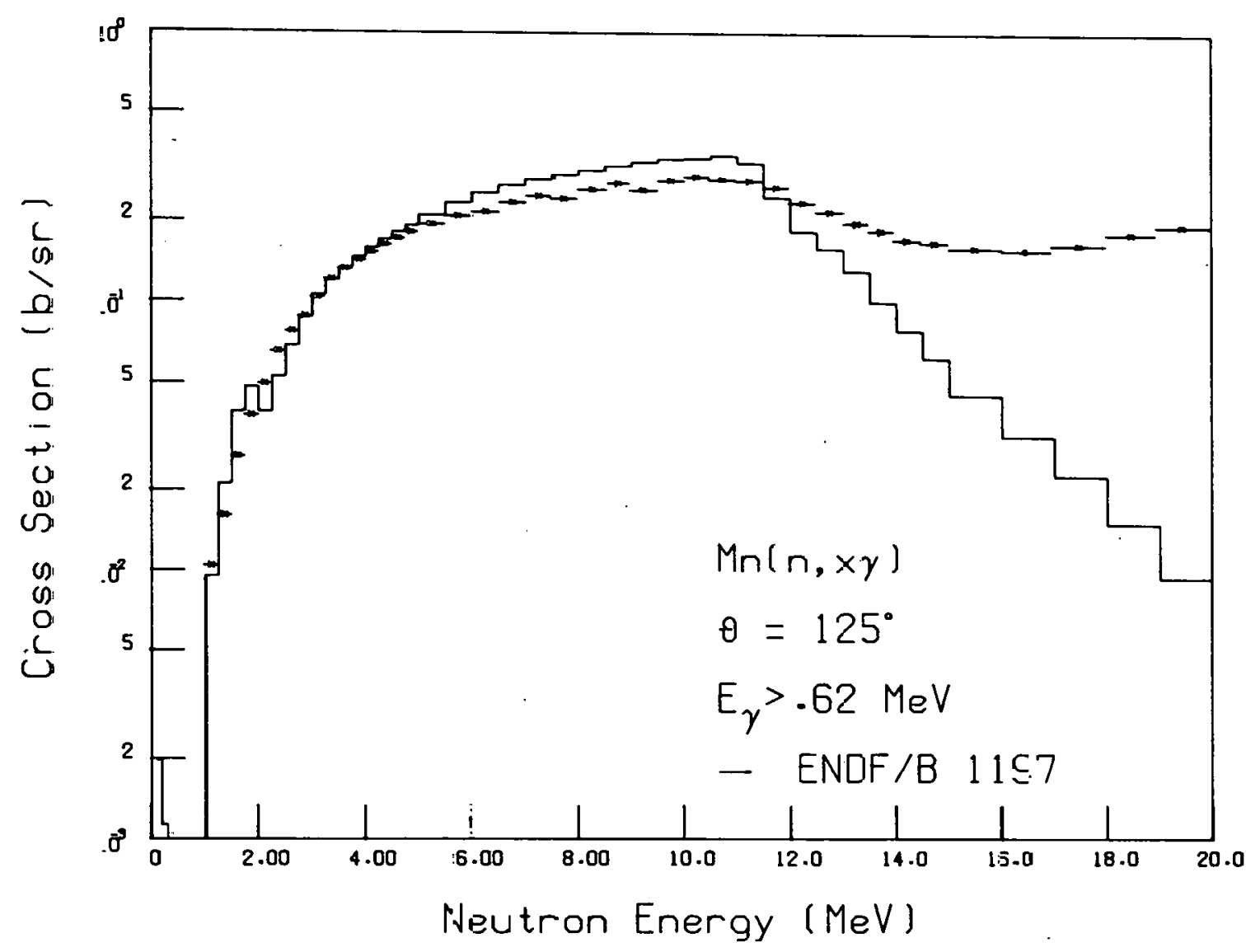

FIGURE 23 


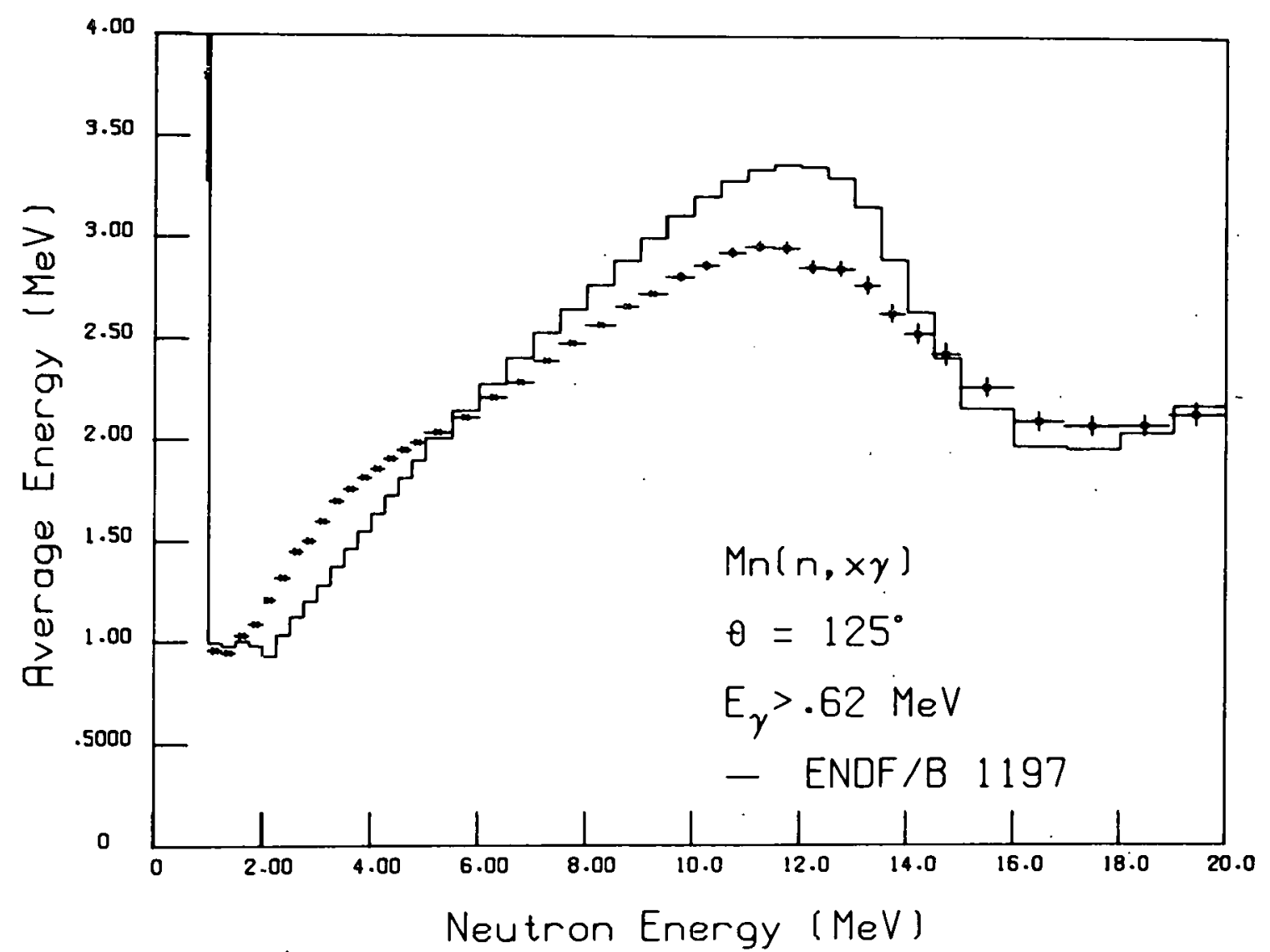

FIGURE 24 


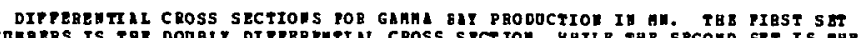

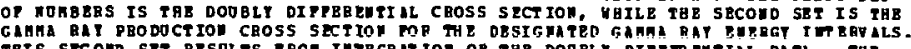
THIS SEC OWD SET BESOLTS PEOA IBTEGB AT ION OP THE DOOBLY DIPYZE EMTHAL DATA. THE

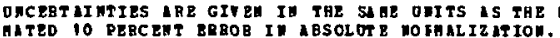

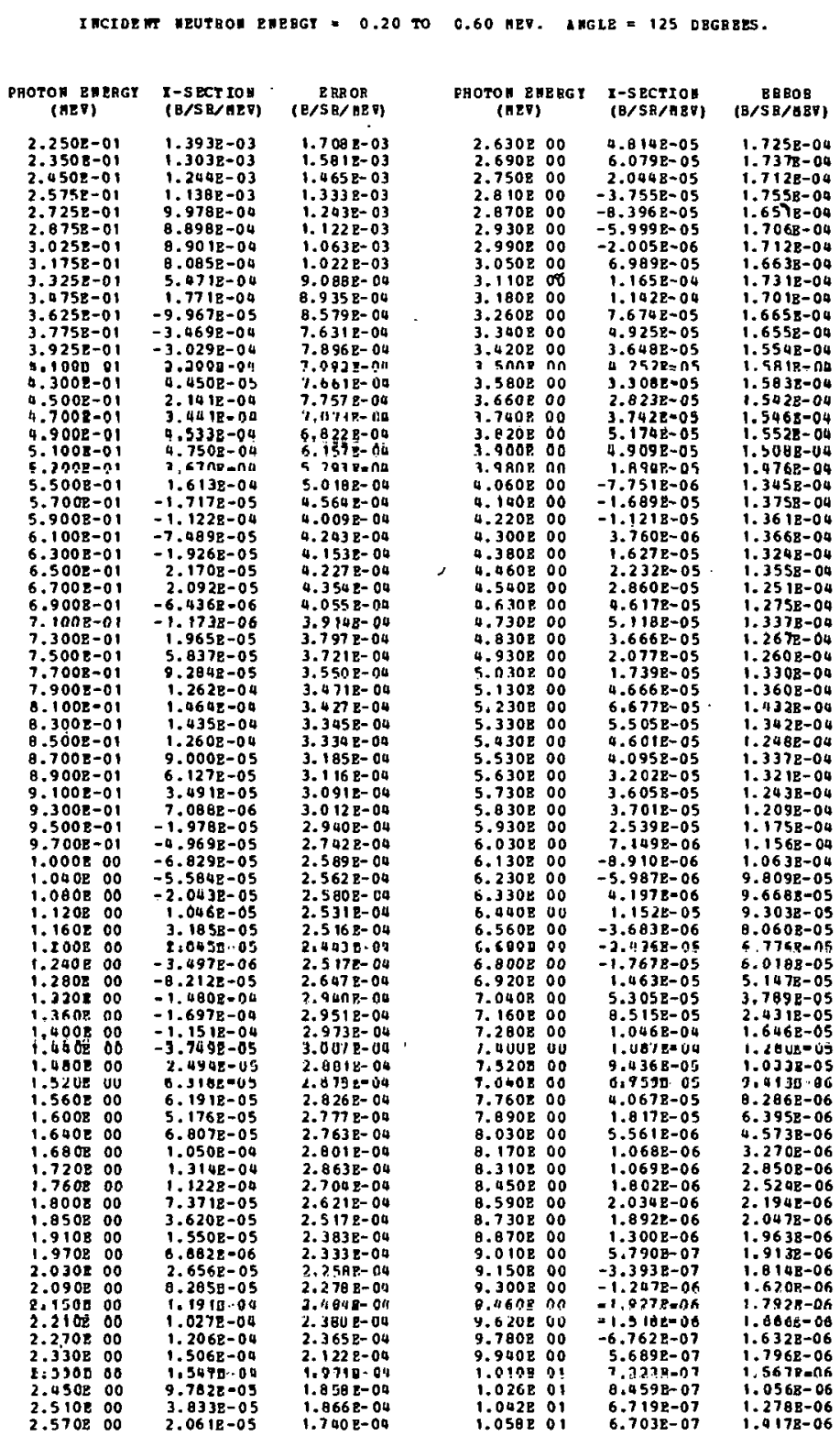

IIT RGRLTPE DITA

\begin{tabular}{|c|c|c|c|c|}
\hline ros 8 & $\begin{array}{r}\text { QWE RG } \\
\text { \{AB }\end{array}$ & If) Integvi & $\begin{array}{l}\text { I-S ECTIOH } \\
(B / S B)\end{array}$ & $\begin{array}{l}\mathrm{ERBOR} \\
(\mathrm{BP} / \mathrm{SB})\end{array}$ \\
\hline 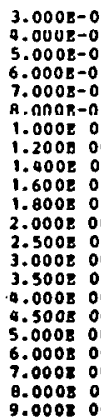 & $\begin{array}{l}000- \\
00\end{array}$ & 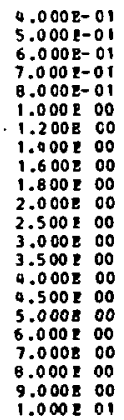 & 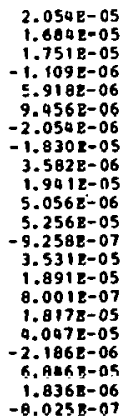 & 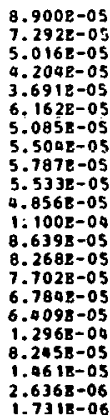 \\
\hline
\end{tabular}




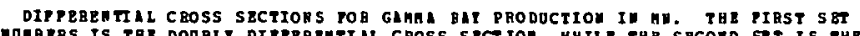

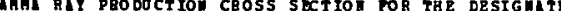

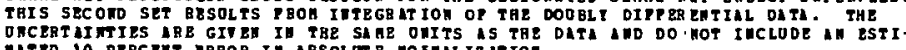

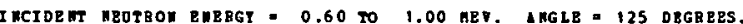

\begin{tabular}{|c|c|c|c|c|c|}
\hline $\begin{array}{l}\text { BOTON ENERGT } \\
\text { (AEV) }\end{array}$ & $\begin{array}{l}7-58 C T \text { IOM } \\
\text { (B/SE/GEV) }\end{array}$ & $\begin{array}{c}\text { ERROR } \\
\mathrm{B} / \mathrm{SR} / \mathrm{MEV} \text { ) }\end{array}$ & $\begin{array}{c}\text { PHOTON } 2 \text { HEB } \\
\text { (MEV) }\end{array}$ & $\begin{array}{l}\text { X-5eCrIon } \\
\text { (B/SB/AEV) }\end{array}$ & $\begin{array}{c}\text { B8BOE } \\
\text { (日/SR/AEY) }\end{array}$ \\
\hline 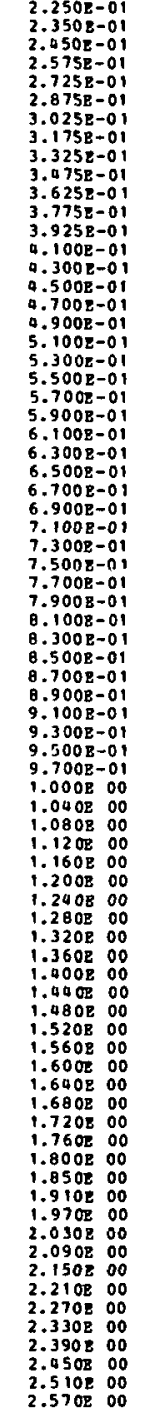 & 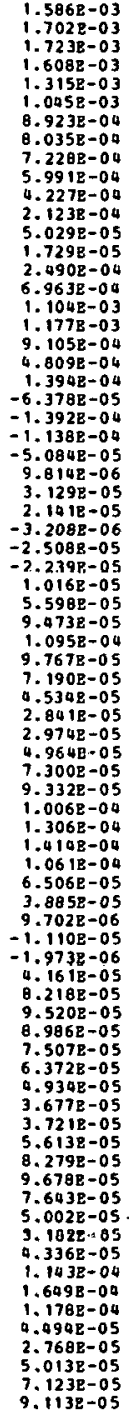 & 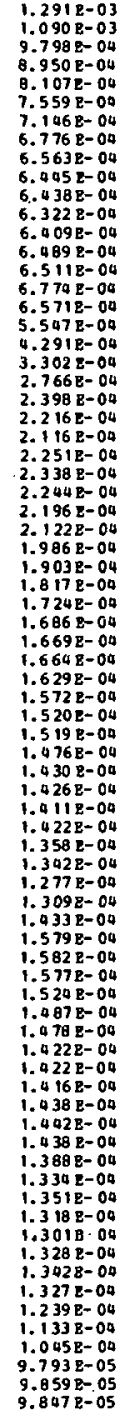 & 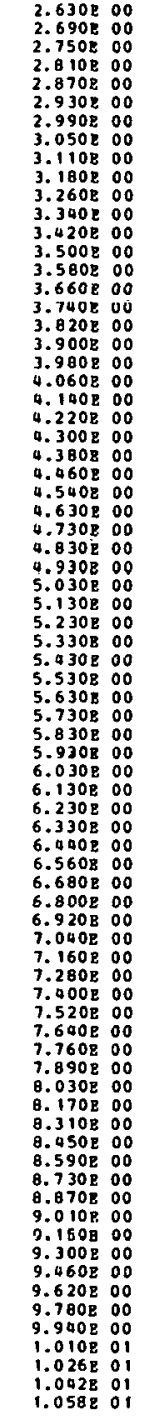 & 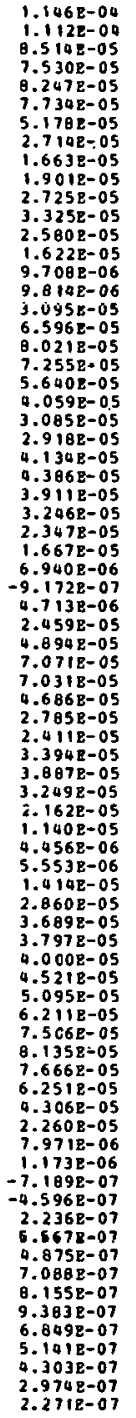 & 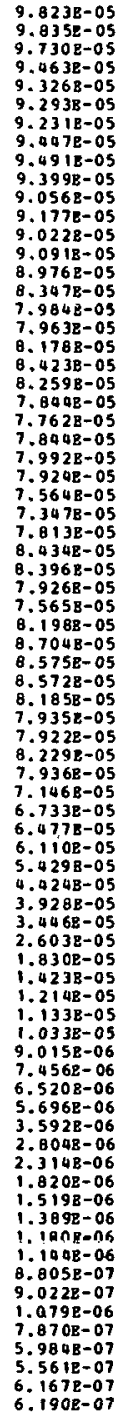 \\
\hline
\end{tabular}

ITEGBATED DAT

\begin{tabular}{|c|c|c|c|c|}
\hline 10005 & $\begin{array}{l}\mathrm{BOB} B \\
\text { (A }\end{array}$ & I I & $\begin{array}{c}\text { X-SECTIOR } \\
\text { (B/SB) }\end{array}$ & $\begin{array}{l}\text { RB80B } \\
(B / 5 B)\end{array}$ \\
\hline 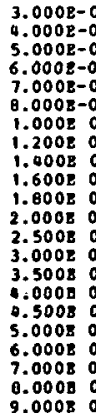 & $\begin{array}{l}01= \\
01= \\
01=1= \\
01= \\
01= \\
01= \\
01= \\
00= \\
00= \\
00= \\
00= \\
00= \\
00= \\
00= \\
00= \\
00= \\
00= \\
00= \\
00= \\
00= \\
00= \\
00= \\
00=\end{array}$ & 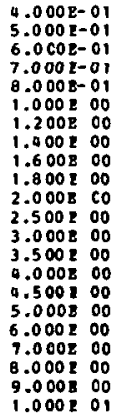 & 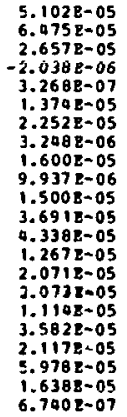 & 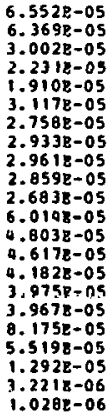 \\
\hline
\end{tabular}




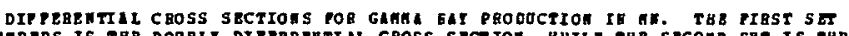

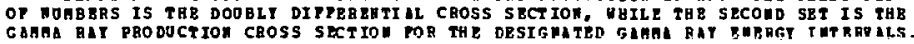

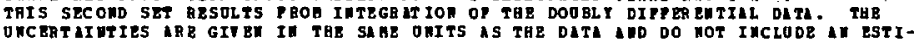

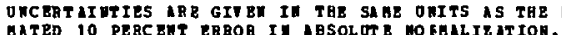

IRCIDET UEUTRON EUEBGY $=1.00$ TO $1.50 \mathrm{MEV}$. ARGLR $=125$ DEGBERS.

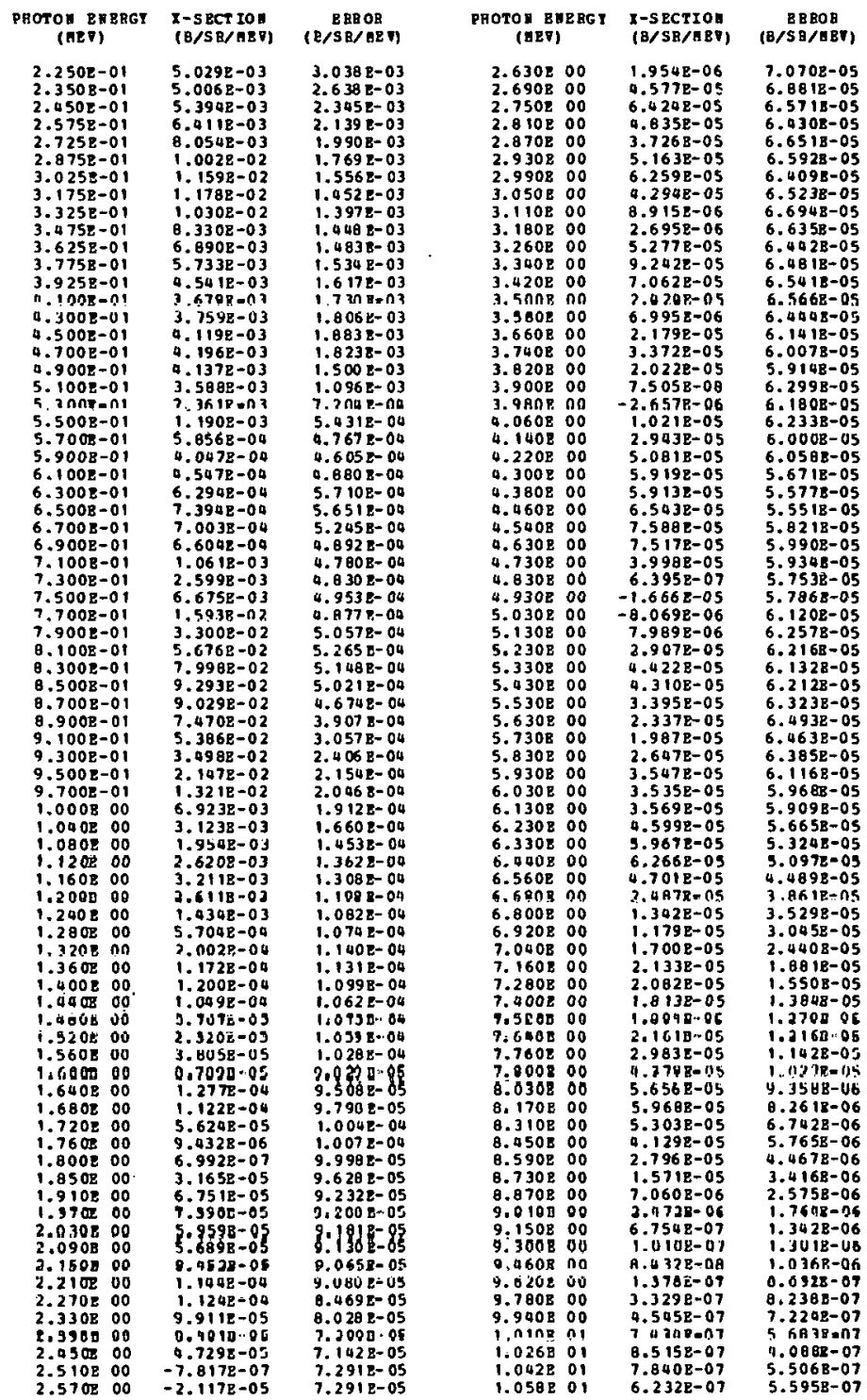

T TTEGRTED DAT

\begin{tabular}{|c|c|c|c|}
\hline $\begin{array}{r}\text { TOH ERBG } \\
\text { FHE }\end{array}$ & II & $\begin{array}{c}\text { X-S RCT ION } \\
(B / S B)\end{array}$ & $\begin{array}{l}\mathrm{REROB} \\
(\mathrm{B} / \mathrm{SB} \mathrm{B})\end{array}$ \\
\hline 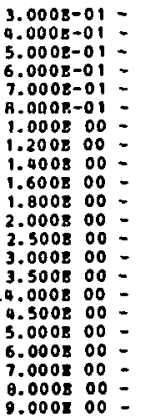 & 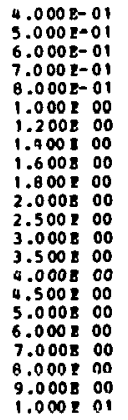 & 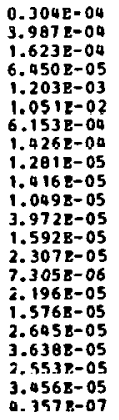 & 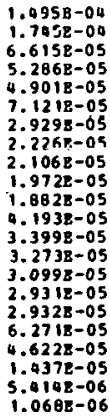 \\
\hline
\end{tabular}




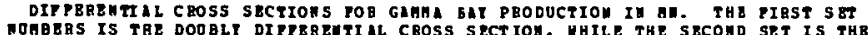
OP WOHEBAS IS THE DOOBLT DIFPRRETIAL CBOSS S ECT ION, UHILE THE SECOND SET IS THB

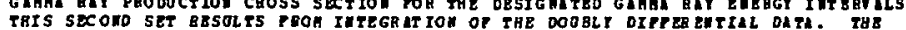

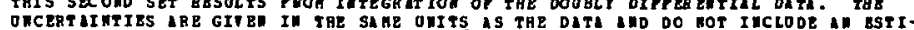

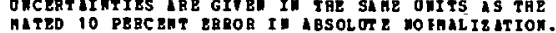

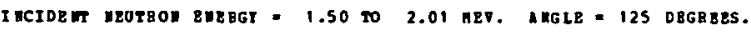

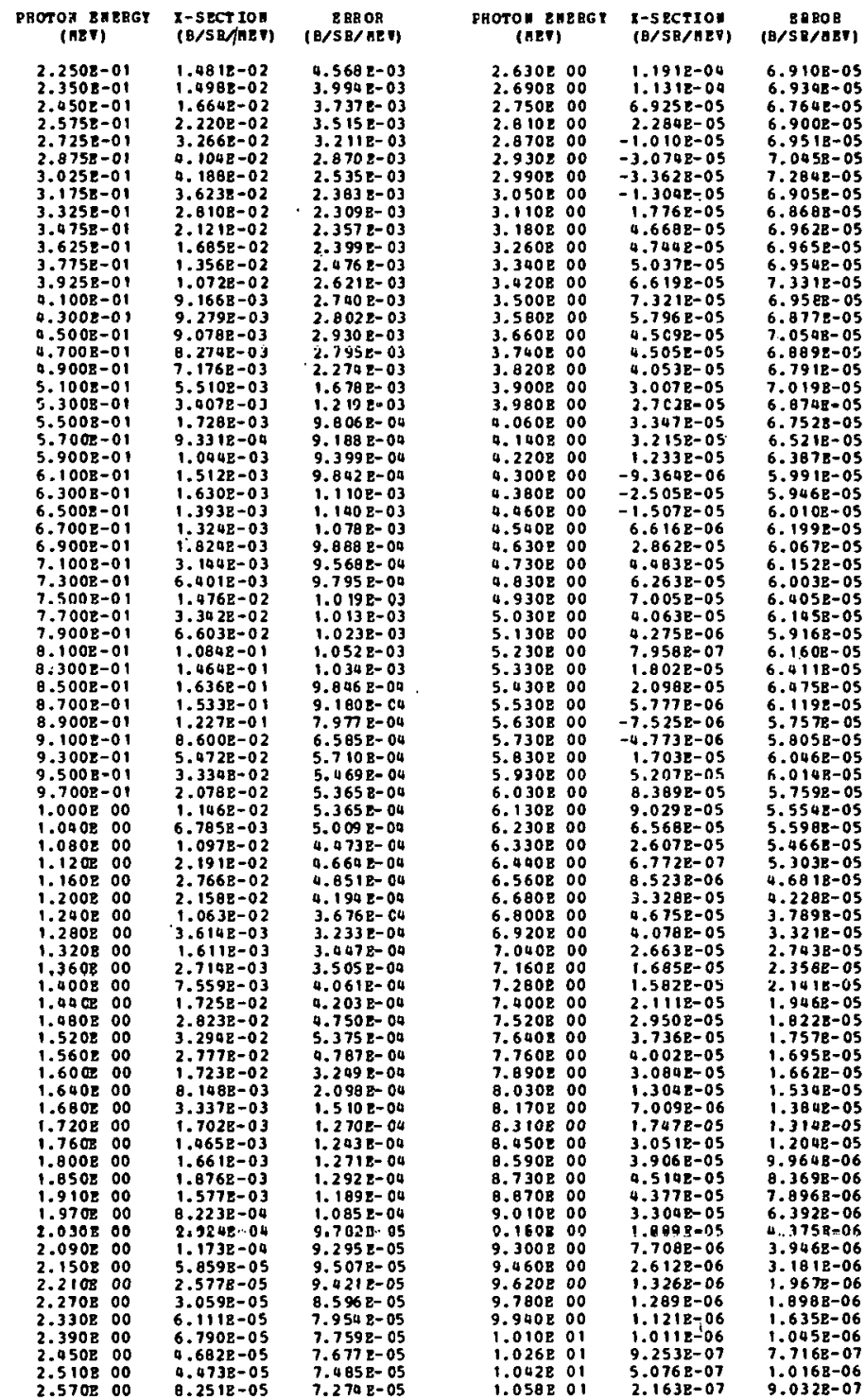

I ITEGATED DATA

\begin{tabular}{|c|c|c|c|c|}
\hline oro & $\begin{array}{l}\text { EAERG } \\
\text { (AER }\end{array}$ & IOTE QV AL & $\begin{array}{c}\text { X-S ECTIOX } \\
\text { (B/SB) }\end{array}$ & $\begin{array}{l}\text { REAOB } \\
(B / S B)\end{array}$ \\
\hline 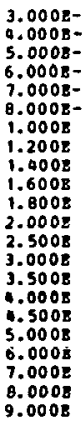 & $\begin{array}{l}-01= \\
011= \\
01=1= \\
01= \\
01= \\
01= \\
01= \\
00= \\
00= \\
00= \\
00= \\
00= \\
00= \\
00= \\
00= \\
00= \\
00= \\
00= \\
00= \\
00= \\
00= \\
00= \\
00=\end{array}$ & 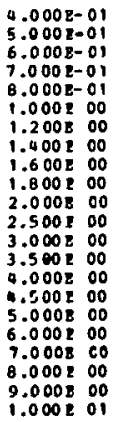 & 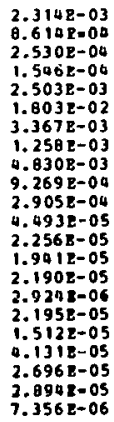 & 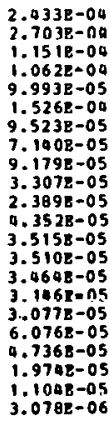 \\
\hline
\end{tabular}




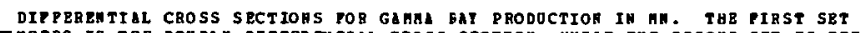

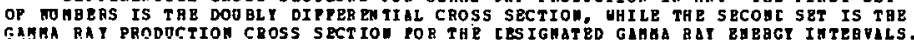

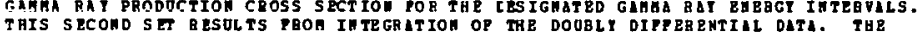

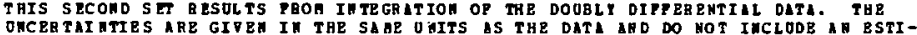
AAT RD 10 PERC ENT PRROR IN ABSOL OTE NORMALIZLTION.

INCIOENT MBOTEON EMERGY $=2.01$ TO 2.52 HEV. ANGLE $=125$ DEgQBES.

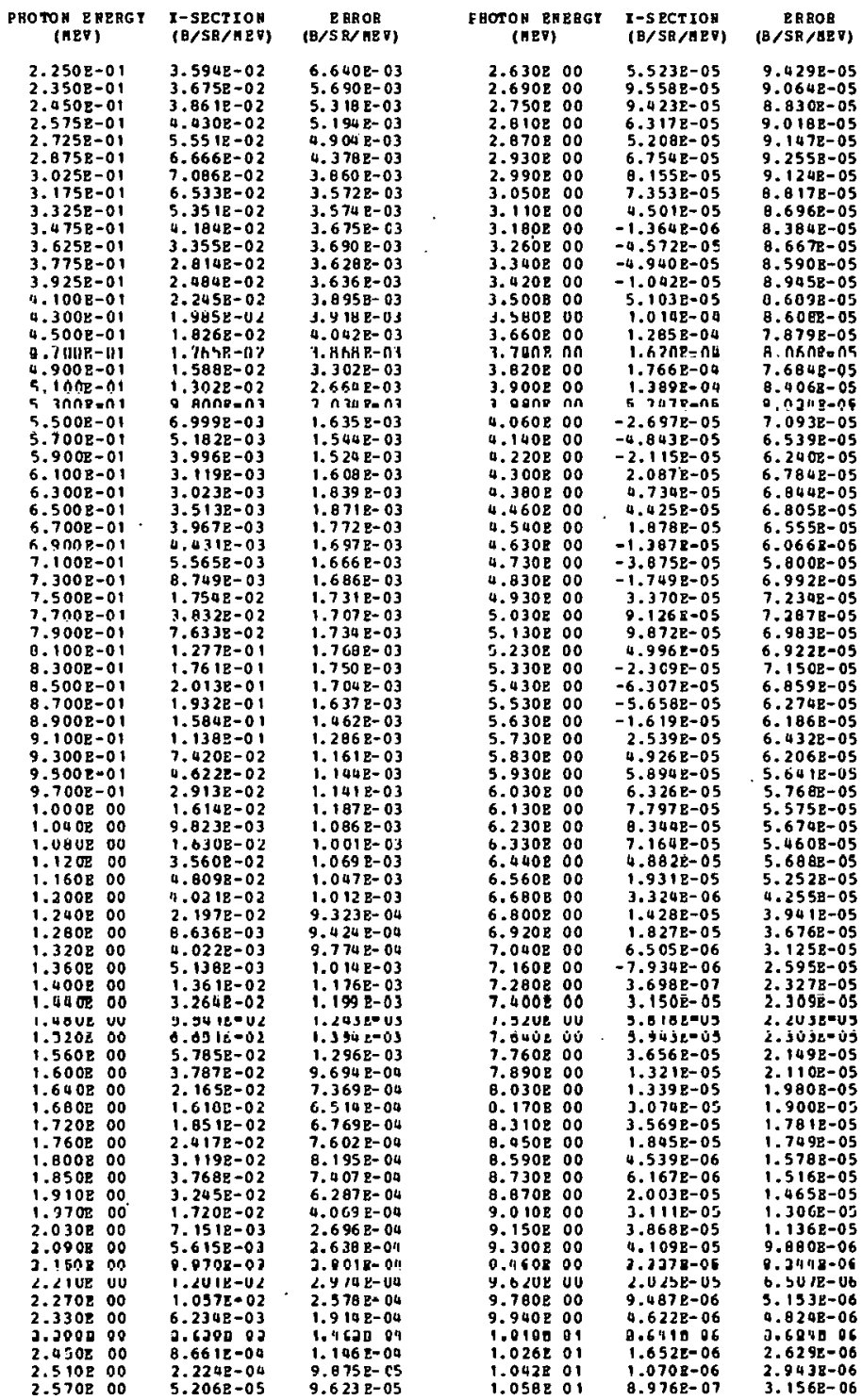

T GT ERARTR Rata

\begin{tabular}{|c|c|c|c|c|}
\hline toron & BNE BC & I IATEAV AL & $\begin{array}{c}\text { I-S ECTION } \\
(\mathrm{B} / \mathrm{SR})\end{array}$ & $\begin{array}{l}\text { ERROR } \\
\text { (B/S B) }\end{array}$ \\
\hline $\begin{array}{l}3.0008 \\
4.0008 \\
5.0008 \\
6.0008 \\
7.0008 \\
6.0008 \\
1.0008 \\
1.2008 \\
1.4008 \\
1.6008 \\
1.8008 \\
2.0008 \\
2.5008 \\
3.0008 \\
3.5008 \\
4.0008 \\
4.5008 \\
5.0008 \\
6.0008 \\
7.0008 \\
8.0008 \\
9.0008\end{array}$ & $\begin{array}{l}-01= \\
-01= \\
01=1 \\
01= \\
01= \\
01= \\
01= \\
01= \\
00= \\
00= \\
00= \\
00= \\
00= \\
00= \\
00= \\
00= \\
00= \\
00= \\
00= \\
00= \\
00= \\
00= \\
00= \\
00=\end{array}$ & 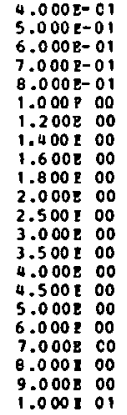 & 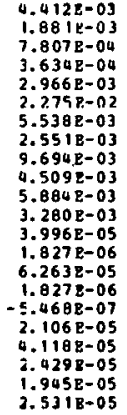 & $\begin{array}{l}3.6508=04 \\
3.7968-04 \\
1.8858-04 \\
1.7598-00 \\
1.7058-04 \\
2.8438-04 \\
2.1178-04 \\
1.9748-04 \\
2.4958-04 \\
1.4778-04 \\
1.2338-04 \\
1.1198-04 \\
4.6228-05 \\
4.3528-05 \\
4.0918-05 \\
3.3798-05 \\
3.2838-05 \\
6.5638-05 \\
4.9298-05 \\
2.3518-05 \\
1.6768-05 \\
0.0468-06\end{array}$ \\
\hline
\end{tabular}


DIPTEREMTIAL CROSS SECTIOHS POR GAHA GAT PRODOCTION IN RM. THE PIRST SET

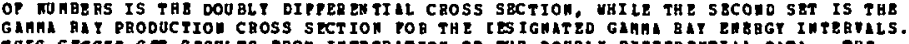

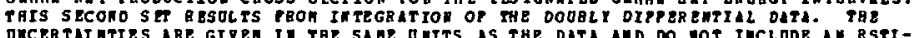

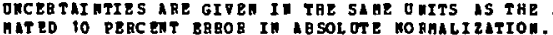

INCTOENT MEUTROA EAERG = 2.52 TO 2.98 AEP. ANGL = 125 DEGBRES.

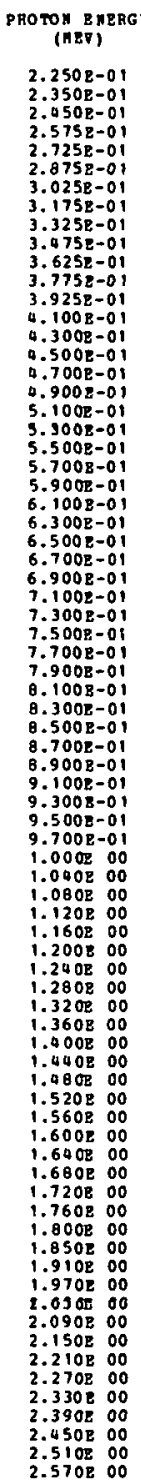

X-SRCTION
$(B / S R / A B V)$

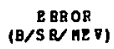

F HOTOM EHBQG
(HEV)

I-SeCTIO)
(B/SR/ARV)

5. $222 \mathrm{P}-02$

9. $3332-03$

5. $3718-02$

$6.0008-0$
$7.1218-0$

$7.663 \mathrm{~B}-03$

$7.403 \mathrm{R}-03$

$2658-0$

6.7558-03

$5.4962-03$
$4.925 \mathrm{E}-03$

$4.9638-03$

$7.0058-02$

$4.9908-0.3$

$1508-02$

(1808-02

$4.7078-03$

5. $046 \mathrm{R}-0.03$

$5082-0$
$.1518-0$

.0248-02

$3.0398-02$

$2.8598-02$
$2.0508-02$

$5.1632-0.3$
$5.2312-03$

$5.0318-63$

$4.318 \mathrm{~B}-0.03$

$2.0358-03$
$2.0048-03$

$.3068-02$

$2.3098-0.3$
$2.338 \mathrm{~g}-0.03$

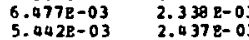

5. $0428-03$

$2.6928-03$

$.2208-03 \quad 2.734 \mathrm{E}-03$

$6.9808-03 \quad 2.585 \mathrm{P}-03$

$1028-02$

$1.6368-02$

$2.4668-02$

$2.432 \mathrm{P}-0.03$

$2.025 \mathrm{E}-03$
$2.405 \mathrm{E}-03$
2.03

$2.4998-03$

1. $3100-0$

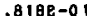

$2.6018-03$

$\begin{array}{ll}1.0958-01 & 2.4768-03 \\ 2.0278-01 & 2.450 \mathrm{~B}-03\end{array}$

$.677 \mathrm{z}-01 \quad 2.215 \mathrm{~B}-03$

$1.2198-0$

1. $980 \mathrm{p}-03$

$1808-02 \quad 1.839 \mathrm{~g}-03$

$.3938-02 \quad 1.8418-03$

i. $8998 \mathrm{~g}-03$

(.4328-02

1. $9448-02$

4. $2278-02$

.. $6058-03$

1.7098- 03

1.650 - 03

$.588-02$
$.5508-02$

$9.4228-03$

$\therefore 696 \mathrm{~g}-0.03$

$2.332 \mathrm{E}-02$
$0.095 \mathrm{E}-02$

$6.212 \mathrm{E}-02$

$1.717 \mathrm{e}-03$
$1.926 \mathrm{~B}-03$
1.962

1.946 1.9283

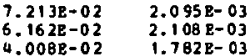

$.008 \mathrm{~B}-02$

$2.3738-02$

i. $5122-03$

$2.2528-02 \quad .5088-03$

$736 \mathrm{E}-0$

i. $6218-03$

$4.4338-02$
$3.7498-02$

$1.689 \mathrm{~B}-0.3$
i. $539 \mathrm{~g}-0.03$

$2.0048-02$

$1.343 \mathrm{E}-03$
$1.020 \mathrm{~B}-03$

$1.155 \mathrm{~B}+02$
$2.1238-02$

$1.0208-03$
$8.7 .798-0.01$
1.967

$9.9532-04$

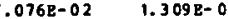

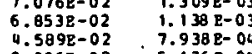

$2.3968-02 \quad 5.136 \mathrm{E}-0.00$

$\begin{array}{ll}1.2708-02 & 3.4638-04 \\ 9.388-03 & 3.0338-00 \\ 9.3218-03 & 2.836 \mathrm{E}-00\end{array}$

RBROB
(9/SR/ARV

$2.630800 \quad 6.746 \mathrm{R}-03 \quad 2.597 \mathrm{R}-04$

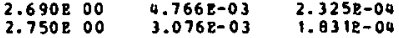

$2.810 \mathrm{E} 00 \quad 1.865 \mathrm{~B}-03 \quad 1.499 \mathrm{~B}-04$

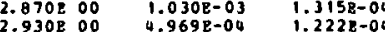

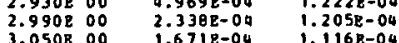

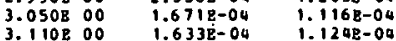

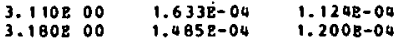

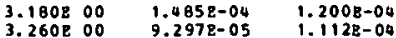

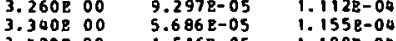

$3.420800 \quad 1.5168-05 \quad 1.1908-00$

$3.500800 \quad-2.3648-05 \quad 9.2058-05$

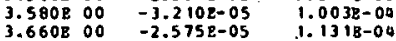

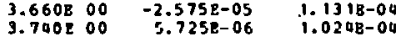

$3.820800 \quad 1.566 \mathrm{E}-05$
$3.0538-04$

$3.900 \mathrm{~B} 000 \quad 3.018 \mathrm{P}-05 \quad \mathrm{~T}, 244 \mathrm{Q}-04$

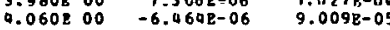

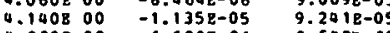

$4.220800 \quad-6.6802-06 \quad 8.5428-05$

$4.300800 \quad 2.866 \mathrm{R}-05 \quad 8.559 \mathrm{~B}-05$

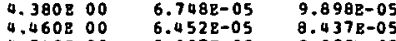

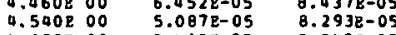

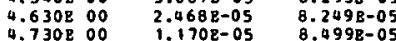

$4.830 \mathrm{~B} 00 \quad 3.700 \mathrm{~B}-06 \quad 7.986 \mathrm{~B}-05$
5.0308 .

4.9308 000 1.2328-05 $8.11 .8 \mathrm{~B}-05$

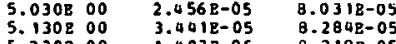

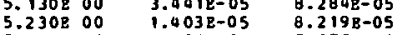

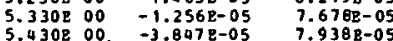

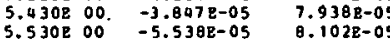

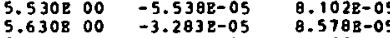

$5.730000 \quad-1.8008-06 \quad 8.6008-05$

$5.830800 \quad 1.354 \mathrm{E}-05 \quad 8.704 \mathrm{E}-05$

6.0308 oo $-8.9018-06 \quad 7.3138-05$

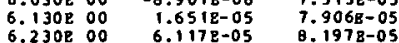

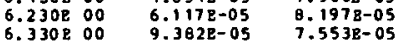

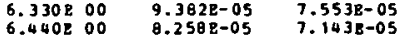

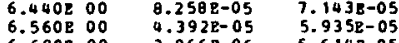

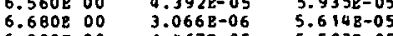

$6.800 \mathrm{E} 00 \quad-1.4678-05 \quad 5.5038-05$

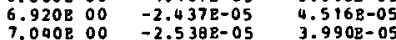

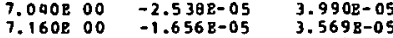

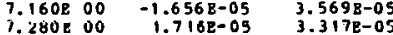

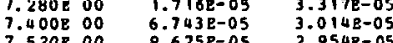

$7878-05$

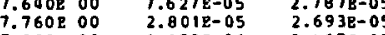

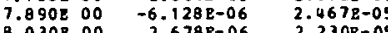

$\begin{array}{lll}8.030800 & 2.678 \mathrm{P}-06 & 2.230 \mathrm{~B}-05 \\ 8.170 \mathrm{~B} 00 & 3.012 \mathrm{~B}-05 & 2.102 \mathrm{~B}-05 \\ 8.05 & 2.05200\end{array}$

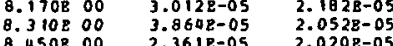

$8.450800 \quad 2.3618-05 \quad 2.0208-05$

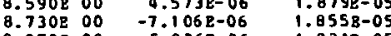

$8.070800 \quad-5.836 \mathrm{~B}-06 \quad 1.821 \mathrm{R}-05$

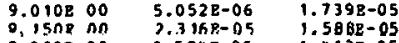

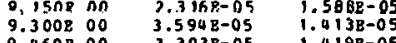

$9.60800 \quad 3.393 \mathrm{~B}-05 \quad 1.419 \mathrm{~B}-05$
$0.738-05$

$\begin{array}{lll}9.620 \mathrm{~B} 00 & 2.473 \mathrm{~B}-05 & 1.16 \mathrm{BB}-05 \\ 9.780 \mathrm{~B} 00 & 1.735 \mathrm{~B}-05 & 8.933 \mathrm{~B}-06\end{array}$

$9.940800 \quad 1.1948-05 \quad 8.818 \mathrm{~B}-06$

$1.010801 \quad 8.8098-06 \quad 6.9128-06$

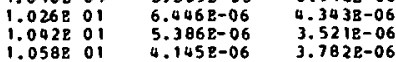

$3.7828-06$

I HTEGATED DATA

\begin{tabular}{|c|c|c|c|}
\hline oton & $\begin{array}{c}\text { EagBGi IN } \\
\text { (ERT) }\end{array}$ & $\begin{array}{c}2-\text { SgC TI of } \\
(B / \mathrm{SB})\end{array}$ & $\begin{array}{l}\varepsilon \text { ERQB } \\
\text { (B/SB) }\end{array}$ \\
\hline 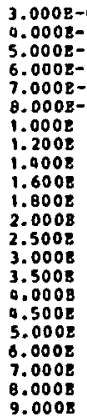 & 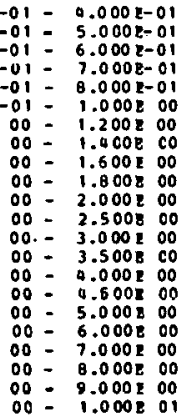 & 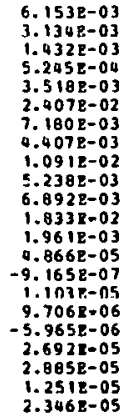 & 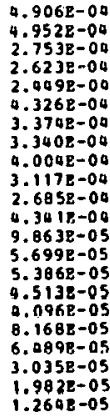 \\
\hline
\end{tabular}




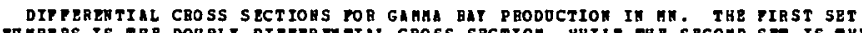
OP ROMBERS IS THE DOU BLY OIPFEB TILL CBOSS SECTION, WHILE THE SBCOND SET IS THE

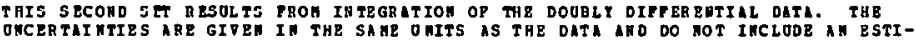
HAT ED 10 PERC EHT ERBOB IN" ABSOL OTE HO RMLLILTIOH.

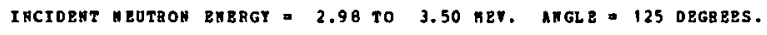

\begin{tabular}{|c|c|c|c|c|c|}
\hline $\begin{array}{l}\text { OTO E EREG I } \\
(A B V)\end{array}$ & $\begin{array}{l}\text { T-SECTIOH } \\
(B / S R / H E V)\end{array}$ & $\begin{array}{c}\text { B RROR } \\
(B / S B / A P V)\end{array}$ & $\begin{array}{l}\text { HOTON BNEBGI } \\
\text { (HEV) }\end{array}$ & $\begin{array}{l}\text { X-SECTION } \\
\text { (B/SB } / A B D)\end{array}$ & $\begin{array}{c}88808 \\
(B / S 8 / A B V)\end{array}$ \\
\hline 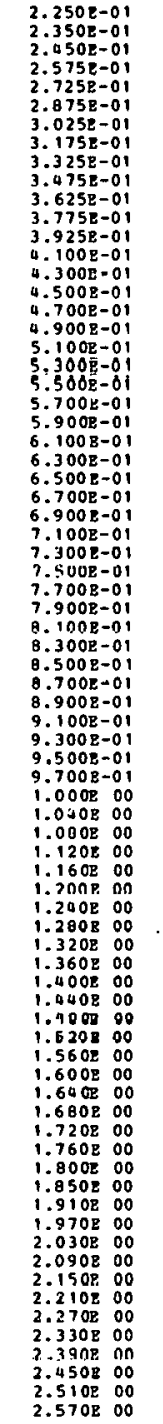 & 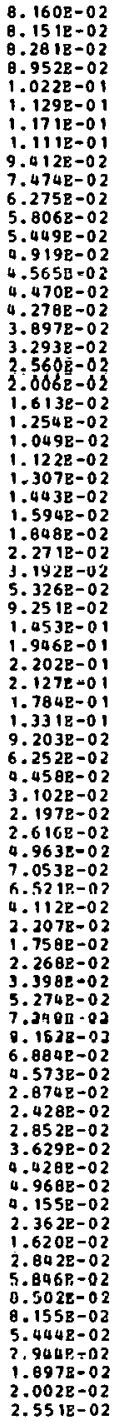 & 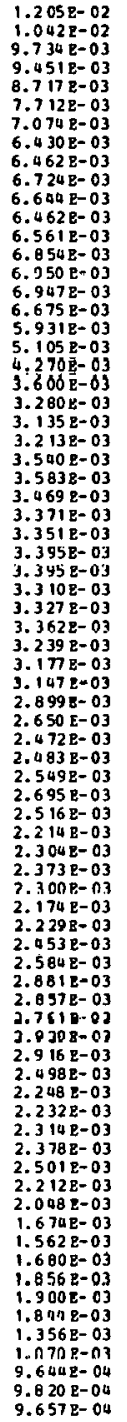 & 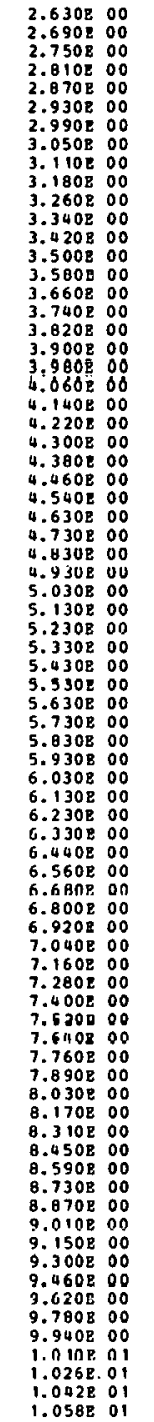 & 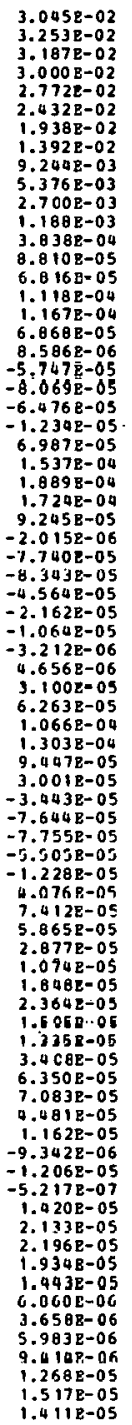 & 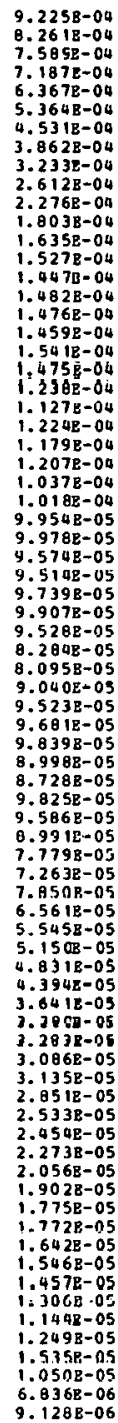 \\
\hline
\end{tabular}

I HTEG RATED DATA

\begin{tabular}{|c|c|c|c|c|}
\hline or or & ENeRC & $\begin{array}{l}\text { GYY IH TE } \\
\text { GPY }\end{array}$ & $\begin{array}{c}\text { I-Sectron } \\
\text { (B/SR) }\end{array}$ & $\begin{array}{l}\text { ERBOR } \\
\text { (B/SB) }\end{array}$ \\
\hline 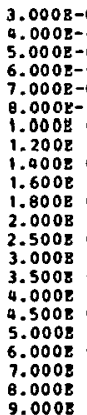 & 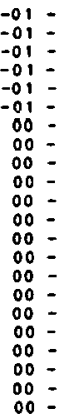 & 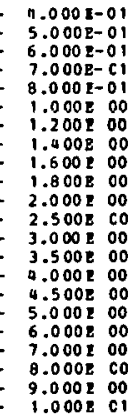 & 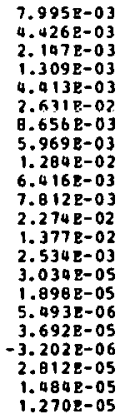 & 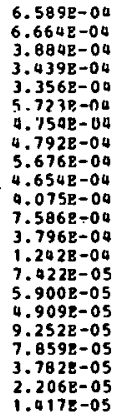 \\
\hline
\end{tabular}




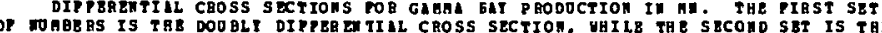

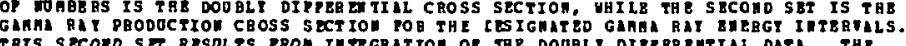

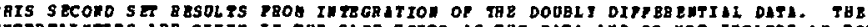

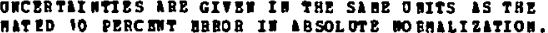

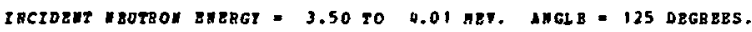

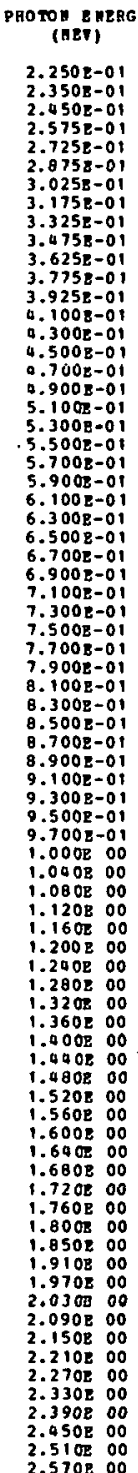

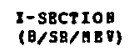

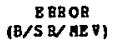

ERoron zheBGr

$\begin{array}{ll}9.1378-02 & 1.5548-02 \\ 9.2018-02 & 1.3022-02\end{array}$

$1.3422-02$
$1.2528-02$
1.23025

$.0168-01 \quad 1.2018-02$

$\left(\begin{array}{ll}3578-0, & \quad, 1028-02\end{array}\right.$

$\begin{array}{ll}1.4738-01 & 8.027 \mathrm{E}-03 \\ 1.0318-01 & 7.905 \mathrm{E}-03\end{array}$

$.2318-01 \quad 7.9218-03$

$\begin{array}{ll}1.0058-01 & 0.088 \mathrm{E}-03 \\ 8.3998-02 & 8.042 \mathrm{z}-03\end{array}$

$8.3788-02$
$8.36399 \mathrm{E}-03$

$6.963 \mathrm{~B}-\mathrm{C2} \quad 8.159 \mathrm{8}-03$

$6.738-02 \quad 7.894 \mathrm{E}-03$

$\begin{array}{ll}6.0568-02 & 7.954 \mathrm{E}-03 \\ -.4388-02 & 0.1158-03\end{array}$

$\begin{array}{ll}5.4388-02 & 8.1158-03 \\ 5.173-02 & 7.6002-03\end{array}$

.

$7.1558-09$
$6.5562-03$

3. $9968-02$

$5.474 \mathrm{E}-0.3$

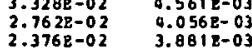

$4.1358-03$

$\begin{array}{ll}2.2128-02 & 4.539 \mathrm{E}-03 \\ 2.6628-02 & 4.363 \mathrm{E}-03 \\ 2.968 \mathrm{C}-02 & 4.092 \mathrm{E}-03\end{array}$

$4.092 \mathrm{z}-03$

$4.0228-03$
$4.036 \mathrm{z}-0.03$

$\begin{array}{ll}3.2418-02 & 4.0368-03 \\ 3.6918-02 & 0.0572-03\end{array}$

$53 \mathrm{~B}-0$
0.02

$4.052 \mathrm{z}-03$
$3.945 \mathrm{E}-0.03$

$3.987 \mathrm{E}-03$

$9.0308-02$
$1.3400-0$

$4.0558-03$

$\begin{array}{ll}1.810 \mathrm{R}-01 & 4.059 \mathrm{R}-03 \\ 2.110 \mathrm{R}-0.1 & 3.982 \mathrm{E}-03\end{array}$

$1238-0$

$3.833 \mathrm{E}-03$

$3.6178-03$
$3.4118-03$

$1.4208-0$

3.140 -03

$7.1108-02$

$3.073 \mathrm{E}-03$

$\begin{array}{ll}5.348-02 & 3.1228-03 \\ 4.0148-02 & 3.3918-03\end{array}$

3.2878-02 3.1508-03

$2.783 \mathrm{~B}-03$
$2.8178-03$

$\begin{array}{ll}5.8538-02 & 2.8178-03 \\ 7.9998-02 & 2.7842-03\end{array}$

2.630800

x-s ECrion

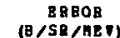

2.690800

$2.810800 \quad 4.5738-02$

2.070800

2.995000

3.050800
3.1108000

3.180800

3. 260800
3.3408 00
3.500800

3.420200

3.500800

3.660200

3.740800
3.8208 00

3.8208
3.9008 00

3.980800
4.060800
4.1408

4.140800
0.220800
4.3008

4.220800
4.300800

4.3008
4.380800

4.460800
0.500800
4.5008

4.630800
4.7308
0.7300

4.730800
8.8308000

4.930800
$\square .930800$
5.9308

4.930800
5.030800

5.130200

5.330800

5.530200

5.630200
5.730200

5.8308 00

5.930800
6.030800
6.2308

6.030200
6.130200
6.300

6,230800

$6.330 \mathrm{~B}$ 00
$6.440 \mathrm{~B} 00$

6.680800

$\begin{array}{ll}7.3298-02 & 2.7328-03 \\ 4.7058-02 & 2.830 \mathrm{E}-03 \\ 2.0248-02 & 2.732 \mathrm{E}-03 \\ 2.3218-02 & 2.985 \mathrm{E}-03\end{array}$

.6808 00

6.920800

7.040800
7.160800

7.1608
7.2808 00

7.400800

7.520200
7.640200

77.7602000

7.890800

8.030800
8.170800

8.1702
9.310800

$8.450 \mathrm{R} 00$

8.5908
8.7300
8.00

8.870800

9.010800

9.300800
9.460800

9.620800

9.940800

1.0108 o
10268

1.022801
1.0582

$1.4748-03$

$308 \mathrm{e}-02$

. $7608-02$

...916z-02

$485 \mathrm{~B}-0$

$1.5278-02$

$9.435 \mathrm{~B}-03$

$6.3228-03$
$3.5668-003$

(5)

$1.6688-03$
$5.0648-04$

. $1662-09$

$5.4998-05$
$5.5838-05$

$2.4382-00$

列

1.998-00

0.307 e- 05

$-1.3198-00$
$-1.0662-00$
-3

$-3.9378-05$
$2.172-05$

$2.172-05$
$6.0808-05$

$6.6708-05$
$8.3028-05$
$0.7092-05$

.

$-4.037 \mathrm{E}-05$
$-1.091 \mathrm{z}-00$

$-0.2668-05$

$4.5238-05$
$1.400 \mathrm{O}-04$
$1.9392-08$

$.696 \mathrm{R}-09$

$0.6388-05$
$1.232-05$

$.6498-06$

$-3.055 \mathrm{R}-05$

$-5.5298-05$
$-5.0058-05$
-5.5028

$-3.562 \mathrm{E}-05$

$7.7942-07$
$4.0322-05$

..210 -05

$5.1958-05$

2.4388-0

$-8.0898-0$

5. $0382-06$

$2.2218-05$

$4.0868-05$

$2.318 \mathrm{R}-05$
$9.1008-06$

$7.616 \mathrm{~B}-06$

$1.0908-05$

$1.3400-05$

$1.49 \mathrm{p}-05$

$1.7138-05$
$1.5992-05$

2.

$2.8318-06$
$4.5728-06$
$0.5319-06$

8.5312-06

$1 \cdot 133 \mathrm{~B}-05$

$1.5098-05$

$1.8982-05$
$1.4748-05$

..2728-

1. $00325-03$

$7.9018-04$

7.1758-04

$6.0578-09$
$5.0408-08$

$4.2298-00$

$3.5768-04$
$3.2758-00$

$2.5098-04$

$2.1908-00$
$1.9645-004$

2.2828-09

$1.9878-04$

$1.9868-00$
$1.6778-00$

1.6778-0

$1.0478-04$

$1.7008-00$

$1.6368-04$
$1.3348-04$
1.515

i. $1578-00$

i. $1568-04$

$1.1058-00$

$1.0868-04$

$1.1298-08$
$1.2798-04$

I. $279 \mathrm{e}-04$

$1548-04$
$1.0308-04$

$1.0948-04$
$1.0358-04$
$1.018-04$

$9.9218-05$

$1.011 \mathrm{E}-0$.
$9.001 \mathrm{~B}-05$

8. $793 \mathrm{Bz}-05$

$8.3908-05$

6.8478-05

$6.5878-05$
$5.9278-05$
$5.093-05$

$3.0942-05$

3. $7003-05$

$3.7008-05$

$3.5008-05$
$3.0303-05$

2.7568-05

$2.3418-05$

1. $8758-05$

$1.7028-05$

$1.4198-05$

1. $3318-05$

1. $3598-95$

$1.4018-05$
$1.3568-05$

$.3568-05$
$i .2118-05$

$1.476 \mathrm{~B}-05$

$1.5208-05$
$1.0698-05$
9.53086

$0.538 z-06$
$9.7318-06$

I UTEG BATED DATA

\begin{tabular}{|c|c|c|c|c|}
\hline oros & 18 & IF & $\begin{array}{c}1-5 E C t I O B \\
(B / S R)\end{array}$ & $\begin{array}{l}\text { BaBOR } \\
(\mathrm{B} / \mathrm{SR})\end{array}$ \\
\hline 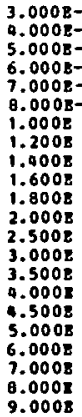 & $\begin{array}{l}-01= \\
-01= \\
00 \\
00 \\
00 \\
00 \\
00 \\
00 \\
00 \\
00= \\
00= \\
00 \\
00= \\
00= \\
00 \\
00= \\
00= \\
00= \\
00= \\
00=\end{array}$ & 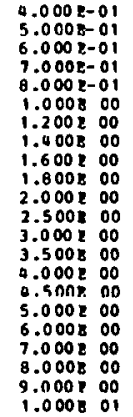 & 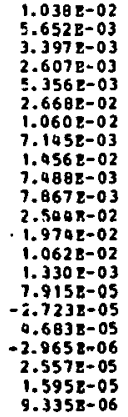 & 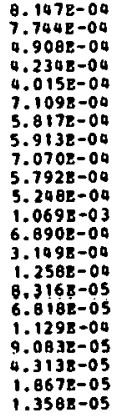 \\
\hline
\end{tabular}




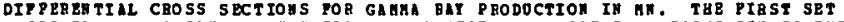

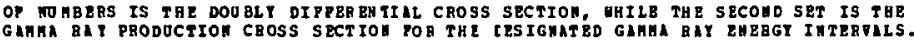

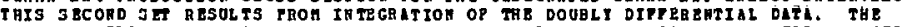

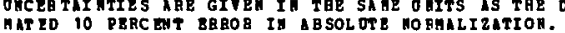

IKCIDERT MEOTROU EMERGT $=4.01$ TO $4.50 \mathrm{BET}$. ANGLE $=125$ DEGBEES.

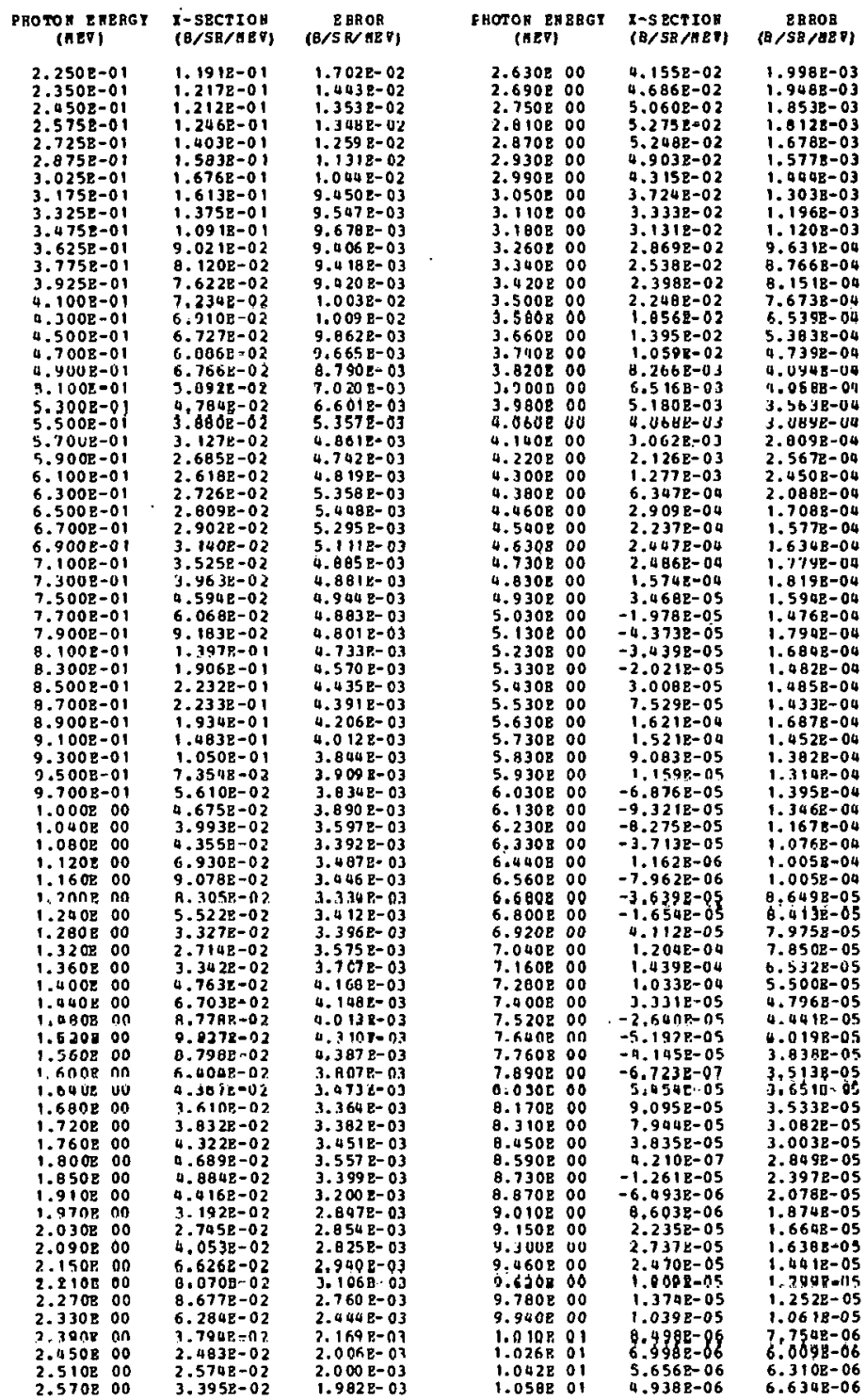

I MTEG RATED DATA

\begin{tabular}{|c|c|c|c|c|}
\hline 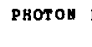 & $\begin{array}{r}\text { ENRRG } \\
\text { (ER }\end{array}$ & If & $\begin{array}{c}\text { - SectroH } \\
(\mathrm{B} / \mathrm{SB})\end{array}$ & $\begin{array}{l}\text { EA8OB } \\
(B / S B)\end{array}$ \\
\hline 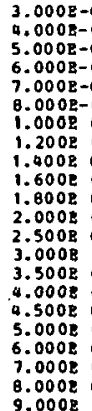 & 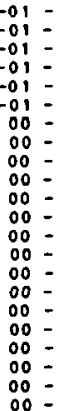 & 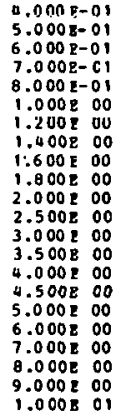 & 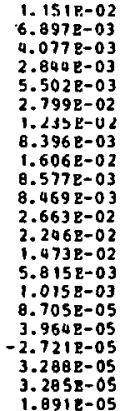 & 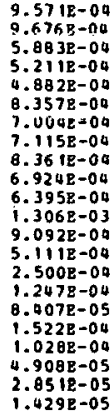 \\
\hline
\end{tabular}




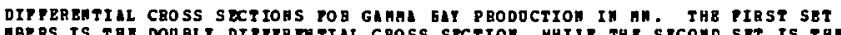

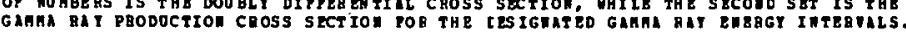

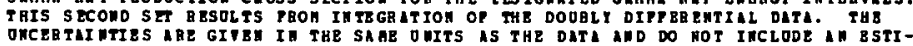

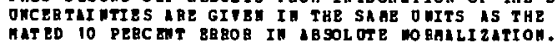

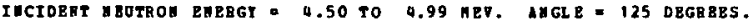

\begin{tabular}{|c|c|c|c|c|c|c|}
\hline $\begin{array}{l}\text { OTON ERERGT } \\
\text { (AEV) }\end{array}$ & $\begin{array}{l}\text { X-SBCTIOB } \\
\text { (B/SB/GEV) }\end{array}$ & $\begin{array}{l}\text { E RROR } \\
\text { JSR }\end{array}$ & $\begin{array}{c}\text { EBoton BN } \\
\text { (HEV) }\end{array}$ & & $\begin{array}{l}\text { x-SECTION } \\
\text { (B/SR/AEV) }\end{array}$ & $\begin{array}{c}\text { ERROB } \\
(B / S B / A B V)\end{array}$ \\
\hline 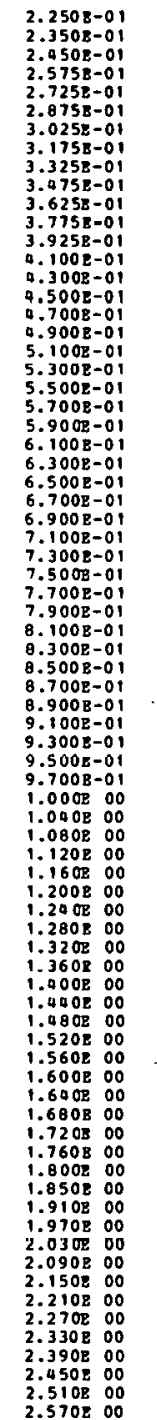 & 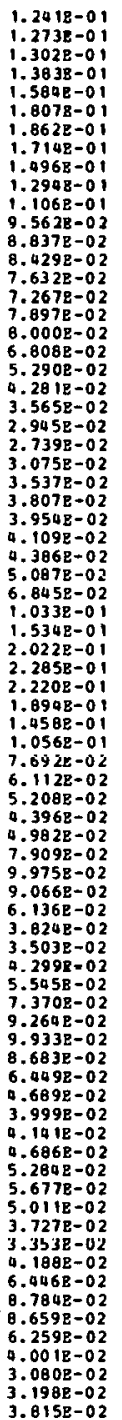 & 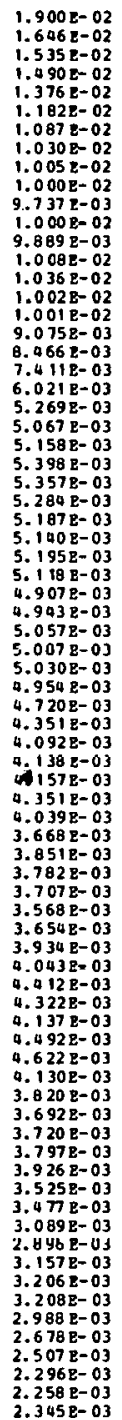 & 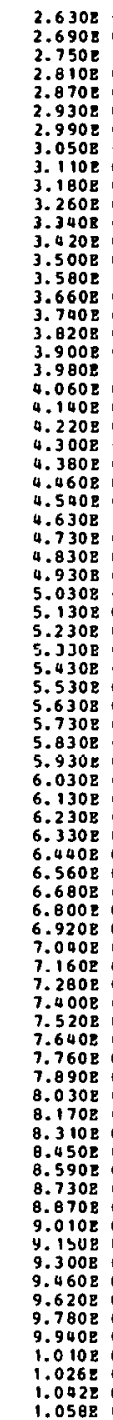 & $\begin{array}{l}00 \\
00 \\
00 \\
00 \\
00 \\
00 \\
00 \\
00 \\
00 \\
00 \\
00 \\
00 \\
00 \\
00 \\
00 \\
00 \\
00 \\
00 \\
00 \\
00 \\
00 \\
00 \\
00 \\
00 \\
00 \\
00 \\
00 \\
00 \\
00 \\
00 \\
00 \\
00 \\
00 \\
00 \\
00 \\
00 \\
00 \\
00 \\
00 \\
00 \\
00 \\
00 \\
00 \\
00 \\
00 \\
00 \\
00 \\
00 \\
00 \\
00 \\
00 \\
00 \\
00 \\
00 \\
00 \\
00 \\
00 \\
00 \\
00 \\
00 \\
00 \\
00 \\
00 \\
00 \\
00 \\
00 \\
00 \\
00 \\
00 \\
00 \\
00 \\
00 \\
01 \\
01 \\
01 \\
01\end{array}$ & 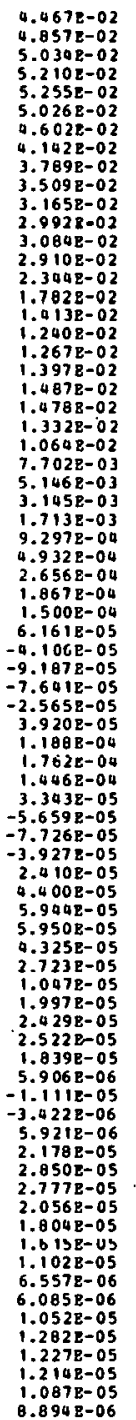 & 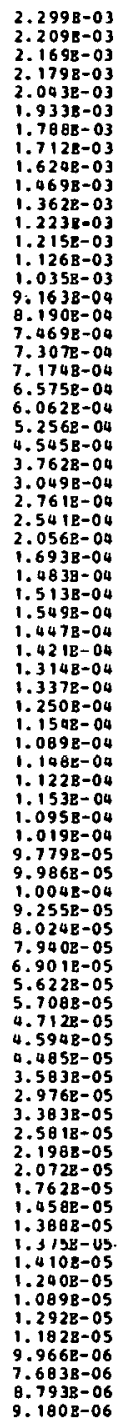 \\
\hline
\end{tabular}

I TTEG RATED DATA

\begin{tabular}{|c|c|c|c|c|}
\hline tron & EN RE & 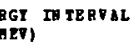 & $\begin{array}{c}1-\text { SECTIOA } \\
\text { (B/SR) }\end{array}$ & $\begin{array}{l}\text { EAEOB } \\
\text { (B/SB) }\end{array}$ \\
\hline 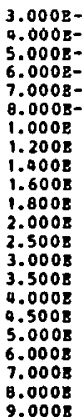 & $\begin{array}{l}-0 \\
-0 \\
-0 \\
-0 \\
-0 \\
-0 \\
00 \\
00 \\
00 \\
0 \\
00 \\
00 \\
00 \\
00 \\
00 \\
00 \\
00 \\
00 \\
00\end{array}$ & 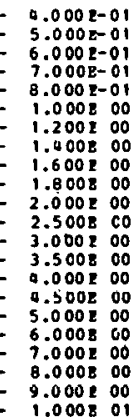 & 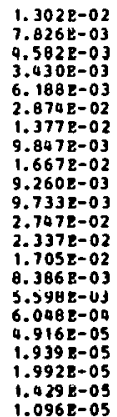 & $\begin{array}{l}1.0088-03 \\
9.9198-04 \\
6.4498-04 \\
5.2808-00 \\
5.0628-04 \\
9.1718-04 \\
7.7318-00 \\
7.6798-04 \\
8.7528-00 \\
1.6048-04 \\
6.8618-04 \\
1.0208-03 \\
1.0748-03 \\
7.0358-04 \\
9.2728-09 \\
2.0628-04 \\
1.0308-00 \\
1.3158-04 \\
9.9868-05 \\
5.2638-05 \\
2.2708-09 \\
1.2758-05\end{array}$ \\
\hline
\end{tabular}


DITPEREATIAL CROSS SBCTIOUS POR GA HAL GAY PBODOCTIOH IU AN THB TIRST SET

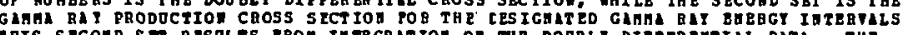

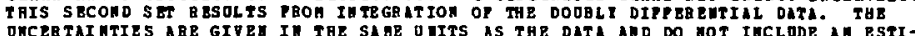
OKCEBTAI HTIES ABE GIVE IN TAE SAME OIITS AS THE OATA AND DO NOT IMCLODE AN ESTI

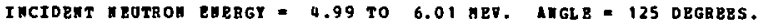

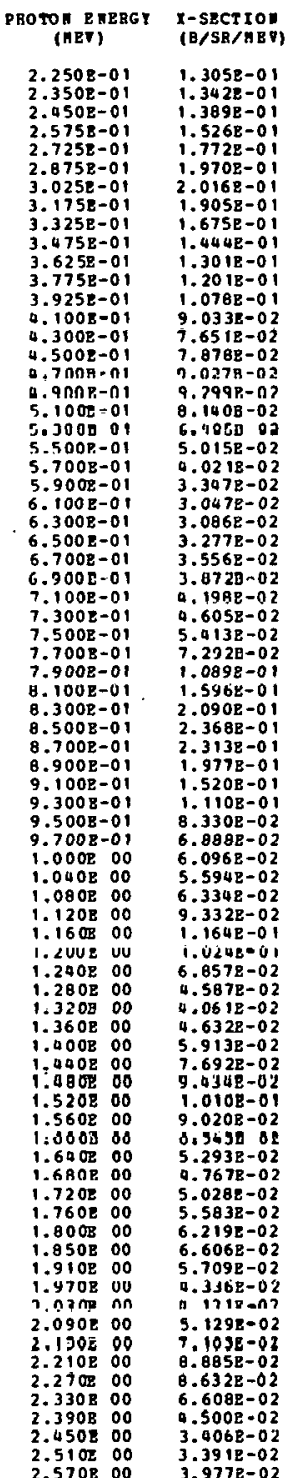

REROR
(B/S\& AEV)

PBOTOM EREBGT
(IBV)

I-S BCTIOS

BRBOR

$2.044 \mathrm{E}-02$
$1.757 \mathrm{E}-02$

$0.656 \mathrm{E}-02$

$2.630 \mathrm{~B} 00$
$2.690 \mathrm{E} 00$
$2.750 \mathrm{E}$
00

$5.154 \mathrm{E}-02$
$5.403 \mathrm{E}-02$

$5.4298-02$

$1.5902-02$

$2.810 \mathrm{~B} 00$

2.930800

$3.050 \mathrm{P} 00$

$3.110 \mathrm{E}$
300
3.1808

3. 180800

$5.1178-02$

$4.201 \mathrm{E}-02$

$1.0668-02$

$1.043 \mathrm{R}-02$

$1.002 \mathrm{R}-02$

$1.009 \mathrm{E}-02$

$1.000 \mathrm{E}-02$

i. $140 \mathrm{E}-02$

$1.080 \mathrm{E}-02$

3.340800

3.420800
3.500800

3.5600 B 00

3.660800
3.71108 OO

3.1208 OO
$3.900 \mathrm{E}$

9. $\operatorname{RgSR-0}$

$9.4518-03$
7.358082

$6.396 \mathrm{~B}-03$

$5.456 \mathrm{E}-03$
$5.185 \mathrm{R}-03$

$5.4168-03$

2.098289

$4.140 \mathrm{~B} 00$

$4.220 \mathrm{P} 00$
4.300800

4.300800

4.460800
4.540800

$6.219 \mathrm{~B}-03$

$5.6418-03$

4.630500

4.7300 00

$4.830 \mathrm{P} 00$
$4.930 \mathrm{E} 00$

$5.635 \mathrm{E}-0$

$5.620 \mathrm{P}-03$

$5.407 \mathrm{E}-03$

$5.288-03$

5.030800
5.130800

5.230800

$\begin{array}{ll}3.748 \mathrm{E}-02 & 1.649 \mathrm{~B}-03 \\ 3.515 \mathrm{~B}-03\end{array}$

$1.468 \mathrm{~B}-03$
$3.417 \mathrm{E}-02$

$3.534 \mathrm{E}-02 \quad \because .2778-03$

$3.3098-02$
$2.9158-02$

$\begin{array}{ll}2.295 \mathrm{~B}-02 & 1.000 \mathrm{R}-03 \\ 1.027 \mathrm{R}-02 & 9.6428-04\end{array}$

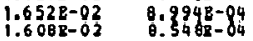

$1.6002-02$

$1.808 \mathrm{z}-02$

$7.4468-04$

$1.992 \mathrm{E}-02 \quad 6.858 \mathrm{BR}-04$

$\begin{array}{ll}6.4278-04 \\ 1.4868-02 & 5.5428-04\end{array}$

$1.251 \mathrm{E}-02 \quad 4.990 \mathrm{~B}-04$

$8.8828-03 \quad 4.7328-04$

$6.752 \mathrm{E}-03 \quad 4.072 \mathrm{E}-04$

$5.569 \mathrm{E}-033.519 \mathrm{~B}-04$

$\begin{array}{ll}4.6078-03 & 3.306 \mathrm{P}-04 \\ 4.0408-04\end{array}$

$2.6958-00$

$\begin{array}{ll}3.0368-03 & 2.4758-04 \\ 2.366 \mathrm{~B}-03 & 2.1758-04\end{array}$

$5.430800 \quad 1.0368-03 \quad 2.1148-04$
$5.530 \mathrm{E} 00 \quad 1.036 \mathrm{E}-03$

$5.630 \mathrm{R} 00 \quad 6.012 \mathrm{~B}-04 \quad 1.920 \mathrm{~B}-04$

$4.927 \mathrm{E}-03$

$4.7148-03$

$4.4618-03$

$4.3108-03$

4. $4098-03$

$4.568 \mathrm{z}-03$

4. $286 \mathrm{E}-03$

$3.828 \mathrm{E}-03$

$3.746 \mathrm{E}-03$

3.170 $8=0$

$3.773 \mathrm{E}-03$

$4.1168-03$

$4.249 \mathrm{E}-03$

$4.559 \mathrm{E}-03$

$.490 \mathrm{E}-0$

$4.938 \mathrm{~B}-03$

$3.913 \mathrm{p}-03$

$3.906 \mathrm{E}-03$

$4.009 \mathrm{R}-03$
$3.946 \mathrm{Z}-03$

$3.998 \mathrm{~B}-03$

$3.642 \mathrm{E}-03$
$3.642 \mathrm{E}-03$

$3.642 \mathrm{E}-03$
$3.392 \mathrm{E}-03$

301 Ren

$3.205 \mathrm{E}-03$
$3.3498-09$

3. 4 i $\mathrm{i} \mathrm{e}-\mathrm{d} \dot{3}$

$3.309 \mathrm{z}-03$

$2.907 \mathrm{E}-03$
$3.566 \mathrm{E}-03$

2. $437 \mathrm{R}-03$

5.730800

$3.6238-04 \quad 1.7718-04$

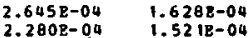

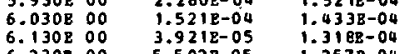

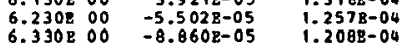

$\begin{array}{lll}6.330 \mathrm{P} 00 & -8.860 \mathrm{~B}-05 & 1.200 \mathrm{BP}-04 \\ 6.440 \mathrm{~B} 00 & -5.369 \mathrm{~B}-05 & 1.020 \mathrm{~B}-04\end{array}$

$6.560 \mathrm{E} 00 \quad 5.011 \mathrm{E}-05$
0.0008

$6.800 \mathrm{R} 00 \quad 9.770 \mathrm{E}-05 \quad 7.582 \mathrm{~B}-05$

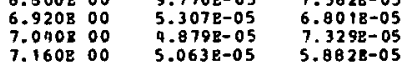

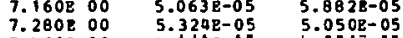

$7.400 \mathrm{E}$ o0 $\quad 4.013 \mathrm{~B}-05 \quad 4.291 \mathrm{~B}-05$

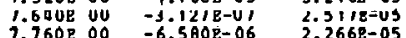

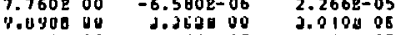

$8.030 \mathrm{R} 00 \quad 1.006 \mathrm{R}-05 \quad 1.811 \mathrm{IR}-05$

$\begin{array}{lll}8.170800 & 2.1148-05 & 1.6938-05 \\ 8.310800 & 1.4618-05 & 1.8428-05\end{array}$

$8.450 \mathrm{~B} 00 \quad 3.244 \mathrm{~B}-05$

$1.7448-05$

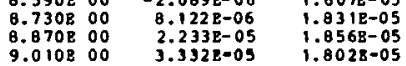

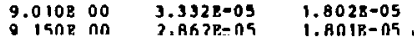

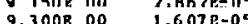

$1.6878-05$

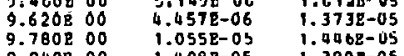

9.9408 oO $1.4082-05$

$1.3908-05$

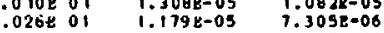

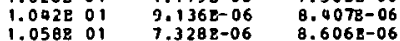

I ITBCDATBD DATA,

PHOTON EHBBGY IN IEQVIL (HEV)

I-SECTION
(B/SB)

$3.000 \mathrm{~B}-01=4.000 \mathrm{z}-01$
$4.000 \mathrm{~B}-01=5.000 \mathrm{z}-01$

$4.000 \mathrm{~B}-01=5.000 \mathrm{E}-01$
$5.000 \mathrm{z}-01=6.000 \mathrm{E}-01$

$6.000 \mathrm{~B}-01=7.000 \mathrm{E}-01$

$.0008-01=8.000 \mathrm{z}-01$

$1.000800=1.200100$

$1.200800=1.400800$

$1.600800=1.600200$

$1.800800=2.000200$

$2.000800-2.500800$

$3.500800-4.000800$

$4.000 \mathrm{E} 00-4.500 \mathrm{E}$ cO

.5002 00 $=5.000200$

$5.000 \mathrm{~B}$ 00 $=6.000 \mathrm{~B}$

$7.000800=8.000000$

$9.000800=9.000200$

$1.4918-0$

$1.0478-03$
$1.065 \mathrm{E}-03$
$5.0038-03$

$6.885 \mathrm{~B}-0.06$

$5.8858-0$
$0.5158-03$

.0308-03 9.

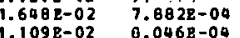

$\begin{array}{rr}.7238-02 & 9.0468-009 \\ .3508-00\end{array}$

$7.9518-04$

.1262-02 $7.2198-09$

$3.9328-02 \quad 0.0788-03$

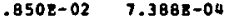

$1.053 \mathrm{z}-02 \quad 0.068 \mathrm{~g}-04$

8.586 E-03 $3.3168-04$

.5988-0 $1.9468-04$

$2.1248-00$

$3.376 \mathrm{E}-05 \quad 3.0608-05$

$\begin{array}{ll}1.2818-05 & 1.7698-05 \\ 1.043 \mathrm{~B}-05 & 1.5700-05\end{array}$ 
DIPPERETTIAL CROSS SECTIONS POB GAMA BAY PBODOCTIOA IN MD. THE PIBST SET

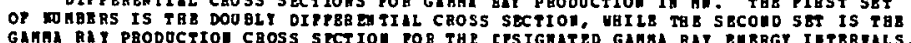

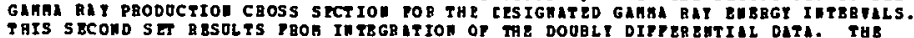

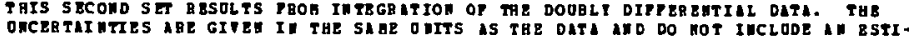

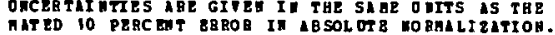

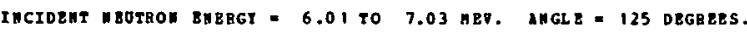

\begin{tabular}{|c|c|c|c|c|c|}
\hline 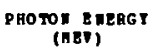 & $\begin{array}{l}x \text {-SBCTIOn } \\
(\theta / S B / A 37)\end{array}$ & $\begin{array}{c}\text { ERROR } \\
\text { (B/S\&/ARV) }\end{array}$ & $\begin{array}{l}\text { PBOTON EREBGt } \\
\text { (HEV) }\end{array}$ & $\begin{array}{l}\text { I-SECTIOW } \\
\text { (B/SB/AEV) }\end{array}$ & $\begin{array}{c}\text { EBBOR } \\
\text { (B/SB/AEU) }\end{array}$ \\
\hline 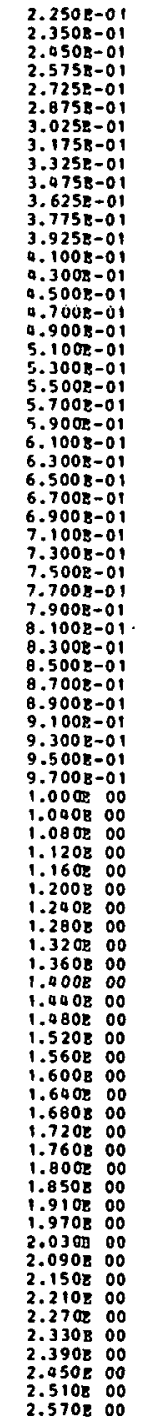 & 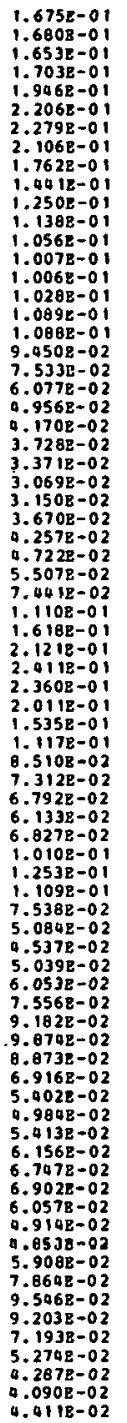 & 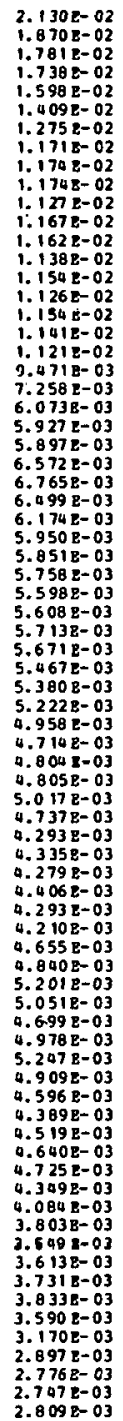 & 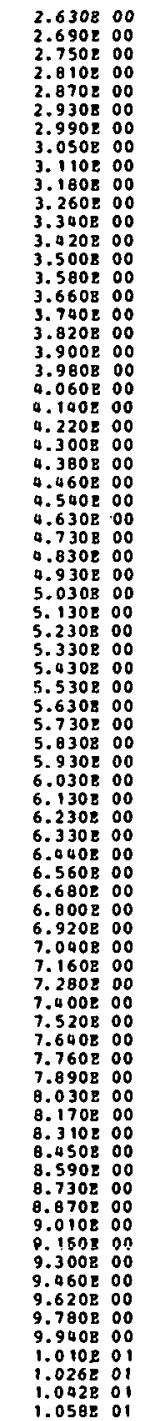 & 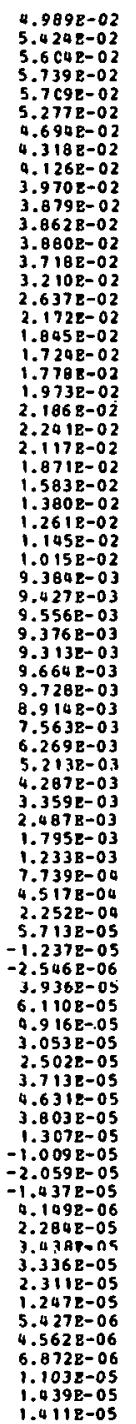 & 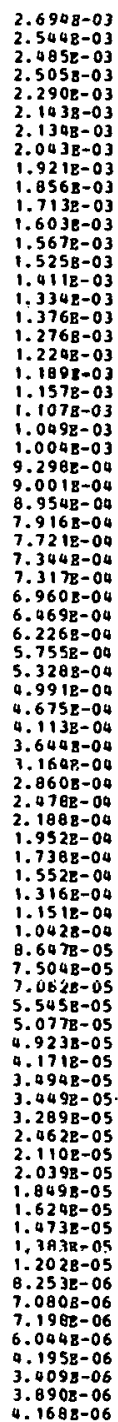 \\
\hline
\end{tabular}

I HTEGATED DATA

\begin{tabular}{|c|c|c|c|c|}
\hline orou & $\begin{array}{l}\text { Ey } 28 G T \\
\text { TAET }\end{array}$ & (n) & $\begin{array}{c}2-58 C \text { Yion } \\
(8 / 5 \mathrm{SB})\end{array}$ & $\begin{array}{l}\text { 88808 } \\
\text { (B/SB) }\end{array}$ \\
\hline 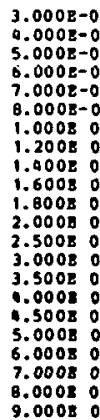 & $\begin{array}{l}00= \\
00= \\
00= \\
00= \\
00= \\
00= \\
00= \\
00=\end{array}$ & 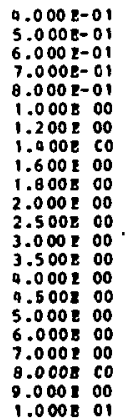 & 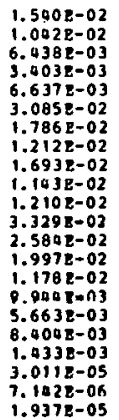 & 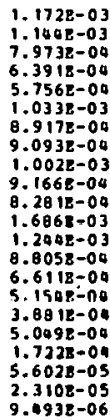 \\
\hline
\end{tabular}




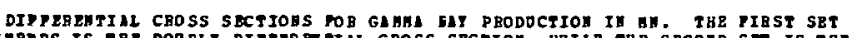
OP WO

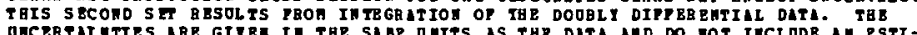

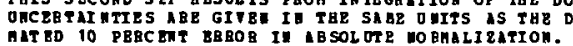

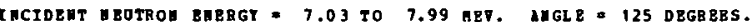

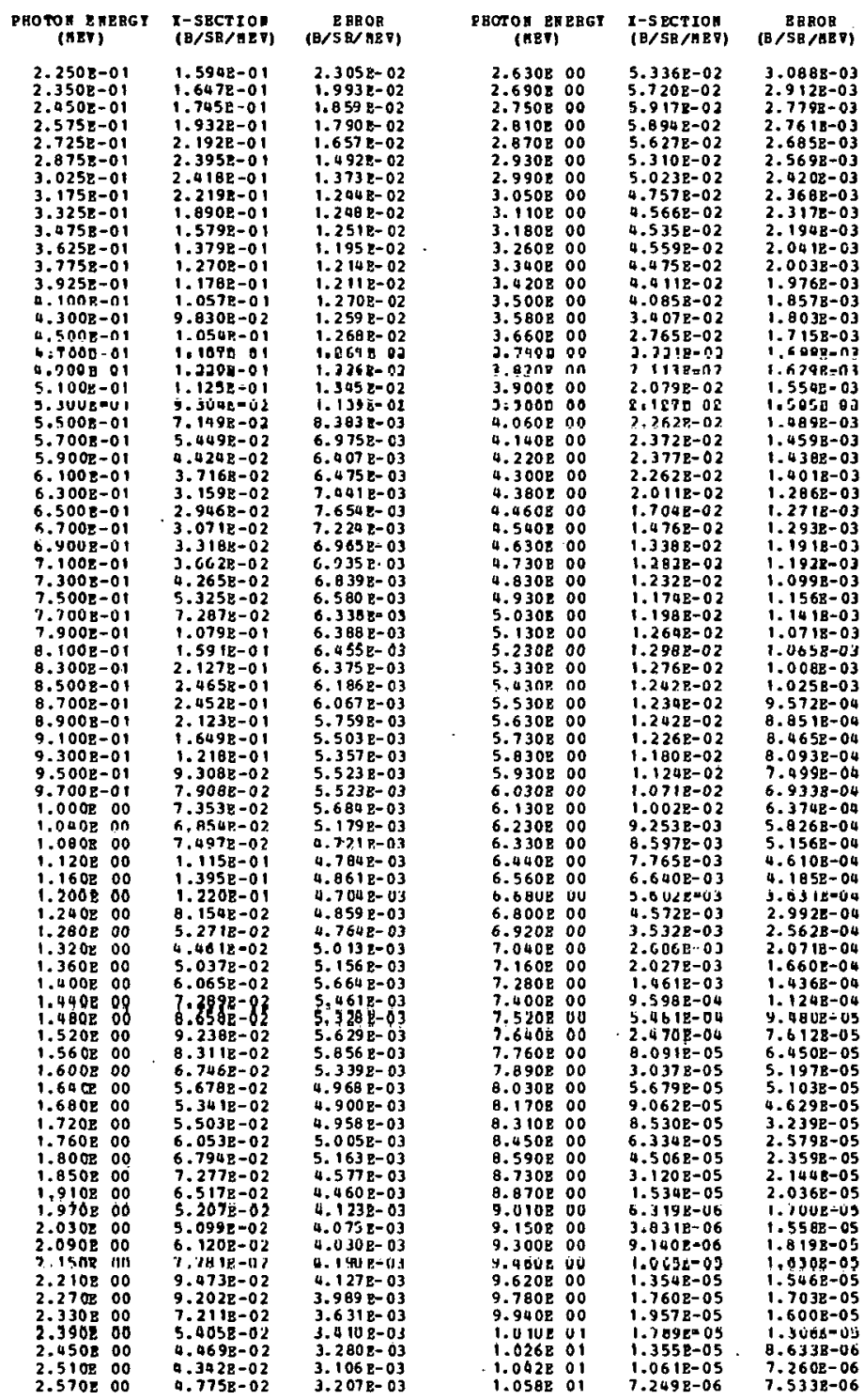

I GTEOATED DATL

\begin{tabular}{|c|c|c|c|c|}
\hline toron & ENE & $\begin{array}{l}\text { BGI III I } \\
\text { BEVI }\end{array}$ & $\begin{array}{c}\text { 1-SEction } \\
(\theta / S R)\end{array}$ & $\begin{array}{l}\text { BRBOB } \\
(B / S B)\end{array}$ \\
\hline 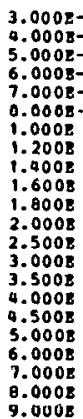 & 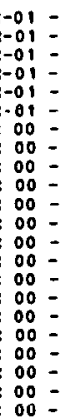 & 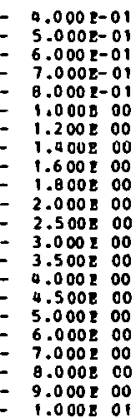 & 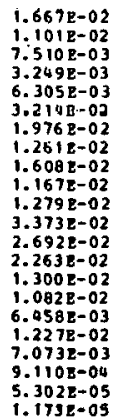 & 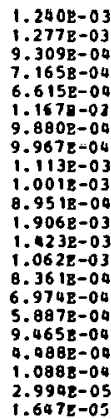 \\
\hline
\end{tabular}




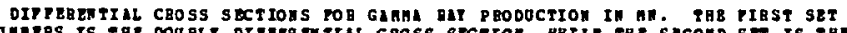

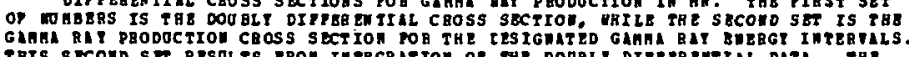

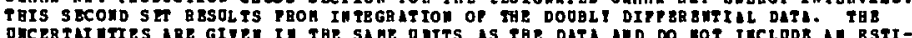

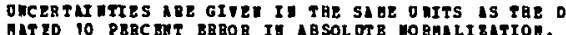

IMCTDEHT N8OTROI EMERGI $=1.9950$ 8.98 MET. ANGLE $=125$ DEGRBBS

\begin{tabular}{|c|c|}
\hline 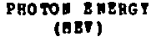 & $\begin{array}{l}\text { X-SECTION } \\
(\theta / S B / H E V)\end{array}$ \\
\hline 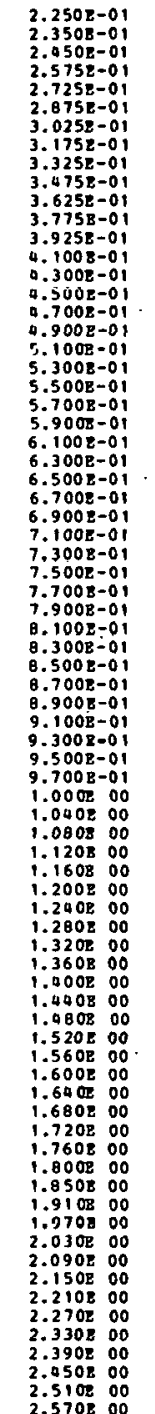 & 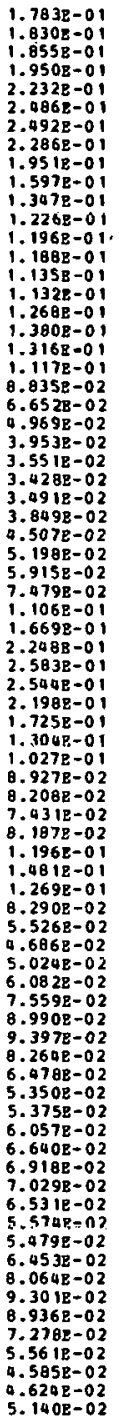 \\
\hline
\end{tabular}

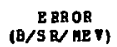

EHORON ENBEG

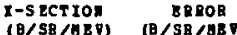

$2.5868-02$
2.2948
2.02

$2.158 \mathrm{z}-0$

$2.059 \mathrm{E}-02$
$1.862 \mathrm{E}-02$

$1.862 \mathrm{E}-02$
$1.612 \mathrm{E}-02$

$1.5218-02$

$1.460 \mathrm{z}-02$

$1.460 \mathrm{E}-02$
1. $370 \mathrm{~g}-02$

$1.370 \mathrm{E}-02$
$1.3318-02$

1. $320 \mathrm{z}-02$

1. $359 \mathrm{R}-02$

1. $360 \mathrm{E}-02$

1.437 E-02

$1.5968-02$

$1.5648-02$
$1.300 \mathrm{E}-02$

$9.0938-03$

$7.5388-03$

$7.0658-03$
$7.1302-03$

$7.760 \mathrm{z}-03$

$7.678 \mathrm{~B}-03$

$7.484 \mathrm{E}-03$

$7.366 \mathrm{E}-03$

$7.1578-03$

$7.108 \mathrm{z}-03$

$.068 \mathrm{p}-03$

$7.070 \mathrm{R}-03$

$6.893 \mathrm{~B}-03$
$6.719 \mathrm{z}-03$

$6.7728-0$

$6.5188-03$

$5.567 \mathrm{E}-03$

5. $778 \mathrm{z}-03$

5. 9 COR- 03
$6.195 \mathrm{R}-03$

$6.195 \mathrm{R}-03$
$5.657 \mathrm{z}-03$

5. $3218-0$

5. $486 \mathrm{E}-03$
5. $3852 \mathrm{z}-03$

5.1912-03

$5.4582-03$
$5.1838-03$

5. $1838-03$

5.6502-03

5. $923 \mathrm{E}-03$

$5.633 \mathrm{Q}-03$

$5.9922-03$
$6.252 \mathrm{z}-03$

$6.252 \mathrm{z}-03$
$5.7948-03$

5. $490 \mathrm{z}-0$

. $306 \mathrm{E}-03$

5. $529 \mathrm{E}-03$

5.900 -03

$5.0718-03$
$4.6812-03$

$4.6812-09$
$9.5078^{-0}-03$

$9.5078-0.93$
$4.570 \mathrm{~B}-0.03$

$4.389 \mathrm{E}-0$

$4.530 \mathrm{~B}-03$

$4.3938-0$

$4.1328-03$
$3.8238-03$

$3.823 \mathrm{E}-03$
$3.644 \mathrm{E}-03$
$3.553 \mathrm{E}-03$

FHOSON
(AE)

\subsection{5}

(1)

2.630200
2.690800
2.7508
2.700

2. 270800

2.990800

3.0508
3,1100

3.180800

3.2608
3.3408 00

3.420800

3.5008
3.500200

3.650800

3.700
3.9200

3.900000

9.060800

4.140800
4.220800

4.300800

4.380800
4.450200
4

4.500800

4.630200
0.7300800
.9308

4.830800

5.930800
5.030800
5.5308

5.1308
500

5.23302 00

5.4308
5.5308 00

5.530200
5.6308

5.6308
5.7300

5.830800

6.030000

$6.130 \mathrm{P} 00$
$6.230 \mathrm{~B} 00$

6.330200
6.440800

6.4408
6.5600
6.600

$6.680 \mathrm{E} 00$

6.800200
6.920800

7.000800

7.1608 00

7.400800

7.520200

$? .760800$

7.890800
8.030800

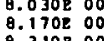

8.310800
8.450800
0.730800

8.4502
8.590000

8.730800
8.870800
0.070800

$9.670 \mathrm{R}$
$9.010 \mathrm{~B}$

9.1508 00

9.460800

9.620200
9.780200

9.780800
9.900800

9.9402 o0

1.0268
1.0028
1.0568

$\begin{aligned} & 5.7778-02 \\ & 5.7298-02\end{aligned} 3108-03$

$5.580 \mathrm{Q}-02 \quad 3.216 \mathrm{~B}-03$

$5.1998-02 \quad 2.8722-03$

$\begin{array}{ll}4.9078-02 & 2.8948-03 \\ 0.8028-02 & 2.8318-03\end{array}$

$.9518-02 \quad 2.5768-03$
0.7618202

$0.761202 \quad 2.4718-03$

$\begin{array}{ll}4.000-02 & 2.5478-03 \\ 3 & .3268-03\end{array}$

0.3078-02 ? 2.2398-03

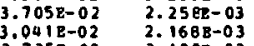

$3.0418-02$

$2.615 \mathrm{~B}-02 \quad 2.033 \mathrm{~B}-03$

$\begin{array}{ll}2.5088-02 & 2.0098-03 \\ 2.5778-02 & 1.9908-03\end{array}$

$2.853 \mathrm{~B}-02 \quad 1.9288-03$

$\begin{array}{ll}2.9708-02 & 1.9578-03 \\ 2.7658-02 & 1.8282-03\end{array}$

2.75502

1.: $2828-03$

2.9608-02 $1.676 \mathrm{~B}-03$

$1.2168-02$
$1.782 \mathrm{E}-02$

$1.4938-02 \quad 1.6458-03$

$1.5838-03$
$1.5168-02$
$1.5188-03$

$1.026 \mathrm{E}-02 \quad 1.543 \mathrm{~B}-0.3$

$\begin{array}{ll}1.515 \mathrm{~B}-02 & 1.5518 \mathrm{~B}-03 \\ 1.5318 \mathrm{R}-02 & 1.480 \mathrm{~B}-03\end{array}$

$1.4168-02 \quad 1.5178-03$

$1.3938-02$
$1.49988-03$

$1.4532-02 \quad 1.402 \mathrm{E}-03$

$\begin{array}{ll}1.445 \mathrm{R}-02 & 1.3658-03 \\ 1.429 \mathrm{~B}-02 & 1.363 \mathrm{~B}-03\end{array}$

$1.4048-02 \quad 1.3228-03$

$1.3642-02 \quad 1.2308-03$

$\begin{array}{ll}.3 \\ 1.346 \mathrm{R}-02 & 1.154 \mathrm{~B}-03 \\ 1.313 \mathrm{~B}-02 & 1.0998-03 \\ 1.0068 & \end{array}$

$1.326 \mathrm{E}-02 \quad 1.066 \mathrm{~B}-03$

$\begin{array}{ll}.315 \mathrm{R}-02 & 1.0018-03 \\ 1.206 \mathrm{~B}-02 & 9.275 \mathrm{~B}-04\end{array}$

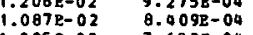

$1.005 \mathrm{E}-02 \quad 7.6938 \mathrm{~B}-04$

$9.0248-03$
$.1998118 R-04$

$9.599 \mathrm{E}-03$
$6.696 \mathrm{~B}-04$

$4.9458-04$

\begin{tabular}{l}
$6.5728-03 \quad 4.4318-04$ \\
\hline
\end{tabular}

$0.6138-03 \quad 3.4098-04$

$\begin{array}{ll}3.5508-03 & 2.9468-04 \\ 2.6078-03 & 2.5978-000\end{array}$

$\begin{array}{ll}2.678-03 & 2.5478-09 \\ 1.908-03 & 2.1228-04\end{array}$

$1.5302-03 \quad 1.7005-04$

$7.8618-00 \quad .0758-00$

$4.530 \mathrm{P}-04 \quad 9.696 \mathrm{~B}-05$

$2.9388-04$
$1.3498-04$$\cdot \begin{aligned} & 7.8948-05 \\ & 7.7678-05\end{aligned}$

.

$3.6788-05$

$1 \because 072=09$

$5.687 \mathrm{E}-05$

$5.8538-05$

$3.5998-05$

$1.648 \mathrm{z}-05$

$1.0198-05$
$1.2978-05$

$9048-05$
$0.998-02$

4. $397 \pi-05$

$.0158-05$
$.0158-05$

$2.0518-05$
2.505

$2.5618-05$

2.3 $325-05$

$1.432 \mathrm{~B}-05$
$8.322 \mathrm{~B}-06$

$1.2878-05$
$1.0258-05$

$8.9258-06$
$8.250 \mathrm{~B}-06$

IATEG TATED DATA

\begin{tabular}{|c|c|c|c|c|}
\hline horol & $\begin{array}{r}\text { ZERG } \\
\text { IHE }\end{array}$ & IN IRQVAL & $\begin{array}{c}I-58 C T I O H \\
(B / S R)\end{array}$ & $\begin{array}{l}B \mathrm{BOB} \\
(\mathrm{B} / \mathrm{SB})\end{array}$ \\
\hline $\begin{array}{l}3.0008-1 \\
4.0008- \\
5.0008- \\
6.0008- \\
7.0008- \\
8.0008- \\
1.0008 \\
1.2008 \\
1.0008 \\
1.6008 \\
1.6008 \\
2.0008 \\
2.5008 \\
3.0008 \\
3.5008 \\
9.0008 \\
9.5008 \\
5.0008 \\
6.0008 \\
1.0008 \\
8.0008 \\
9.0008\end{array}$ & 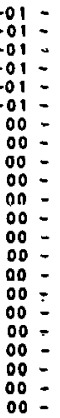 & 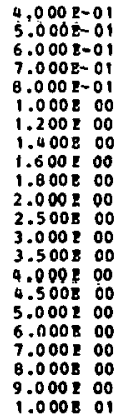 & 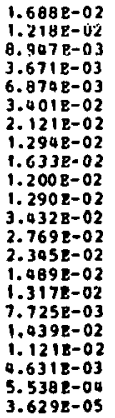 & $\begin{array}{r}1.4158-03 \\
1.4108-03 \\
1.0538-03 \\
7.4938-04 \\
7.0868-04 \\
1.2648-03 \\
1.1088-03 \\
1.0998-03 \\
1.1928-03 \\
1.0978-03 \\
9.7908-04 \\
2.1098-03 \\
1.6338-03 \\
1.2858-03 \\
1.0568-03 \\
9.0008-04 \\
7.9508-04 \\
1.4118-03 \\
8.9058-00 \\
3.6008-04 \\
9.2638-05 \\
3.1988-05\end{array}$ \\
\hline
\end{tabular}




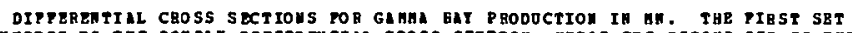

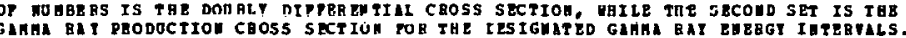
THIS SECONO SET BESOLTS TEOA INTEGR ATION OP THE DOOBLY DIFTEREUTIAI DATA. THB

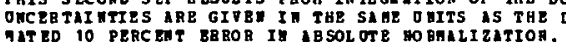

INCIDEHT NBOTRON BNEBGT $=0.98$ IO $10.01 \mathrm{MET}$. AMGLE $=125$ DRGBERS.

\begin{tabular}{|c|c|c|c|c|c|}
\hline $\begin{array}{l}\text { 10 POM EnERG } \\
\text { (AET) }\end{array}$ & $\begin{array}{l}\text { I-SECTIOA } \\
\text { (B/SB/AEV) }\end{array}$ & 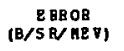 & $\begin{array}{c}\text { FHOTON EHBBC } \\
\text { (ABV) }\end{array}$ & $\begin{array}{l}\text { I-S ECTION } \\
\text { (B/SR/ABV) }\end{array}$ & $\begin{array}{c}\text { ERROR } \\
\text { (B/SB/AEV) }\end{array}$ \\
\hline 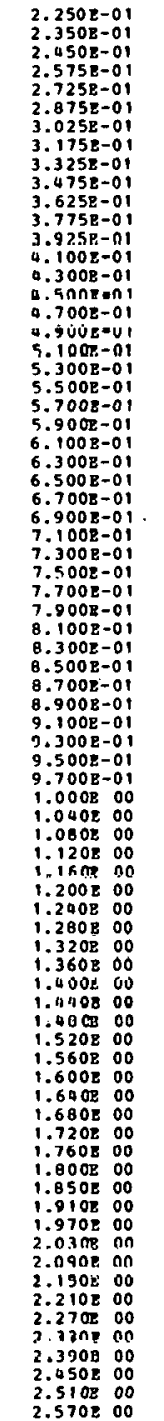 & 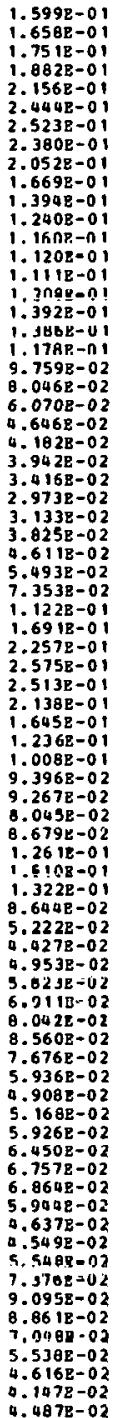 & 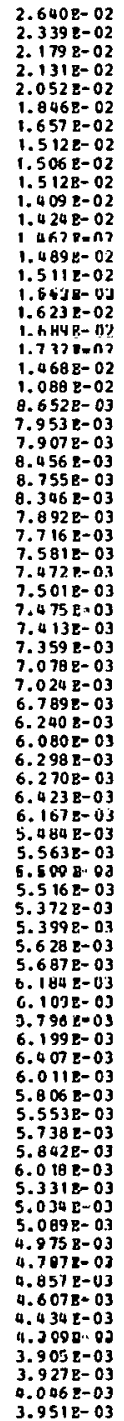 & 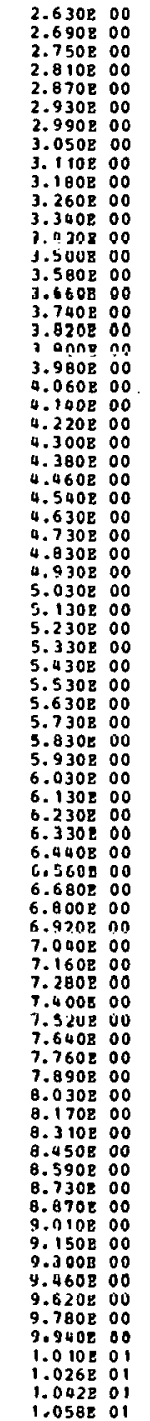 & 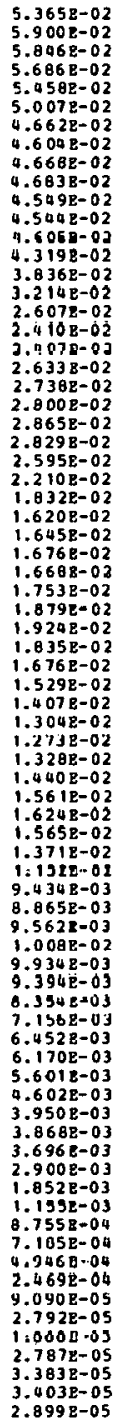 & 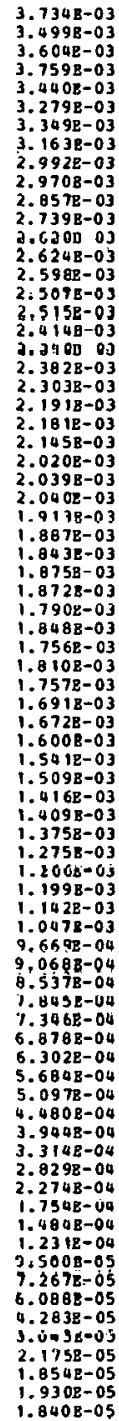 \\
\hline
\end{tabular}

I HTELTED DATS

\begin{tabular}{|c|c|c|c|c|}
\hline 201: & $\begin{array}{l}\text { EMBG } \\
\text { (HE) }\end{array}$ & $\mathrm{y}_{\mathrm{I}}^{\mathrm{TN} \mathrm{TE}}$ & $\begin{array}{c}I-\text { SEctIon } \\
(B / S B)\end{array}$ & $\begin{array}{l}\text { BEROA } \\
\text { (B/SB) }\end{array}$ \\
\hline 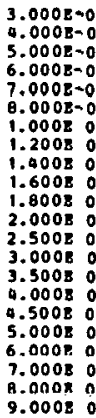 & $\begin{array}{l}00 \\
00 \\
00 \\
00 \\
00 \\
00 \\
00 \\
00 \\
00 \\
00 \\
00 \\
00 \\
00 \\
00\end{array}$ & 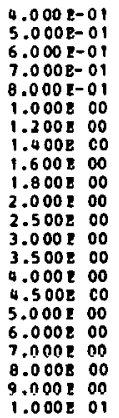 & 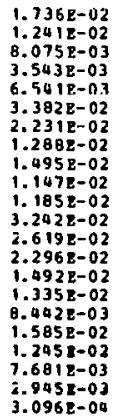 & $\begin{array}{r}1.4878-03 \\
1.5748-03 \\
1.1878-03 \\
8.2808-04 \\
7.5508-04 \\
1.3388-03 \\
1.1508-03 \\
1.1148-03 \\
1.2258-03 \\
1.5568-03 \\
1.0458-03 \\
2.2278-03 \\
1.0108-03 \\
1.9348-03 \\
1.2378-03 \\
1.0778-03 \\
9.5168-04 \\
1.7258-03 \\
1.2608-03 \\
7.4888-04 \\
3.1998-04 \\
7.6588-05\end{array}$ \\
\hline
\end{tabular}


OIPTEREWTIL CBOSS SECTIONS POB GIMAA BAT PRODOCTION IN HN. THB PIRST SET

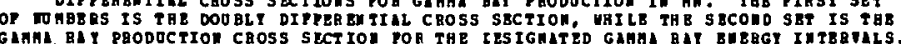

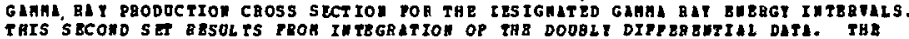

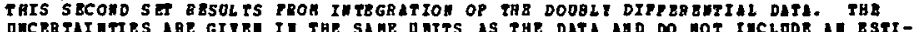

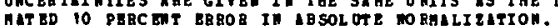

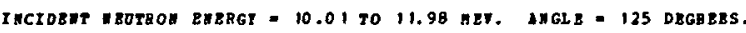

\begin{tabular}{|c|c|}
\hline $8 \mathrm{E}$ & - SEC \\
\hline 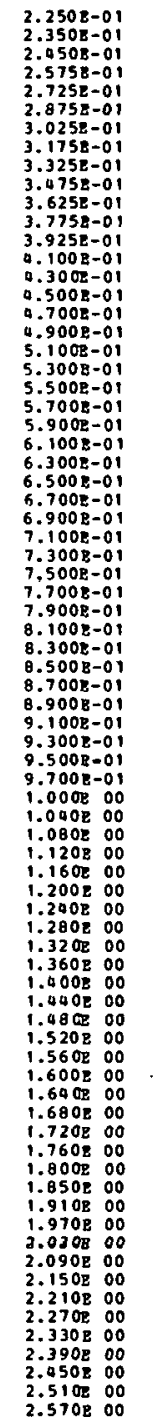 & 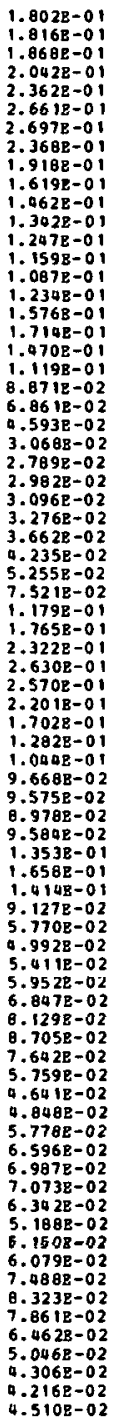 \\
\hline
\end{tabular}

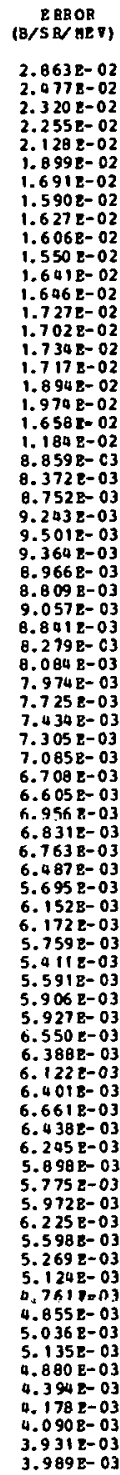

PBoton Ba 2 EGT

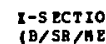

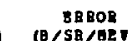

$2.630 \mathrm{~B} O$

$5.0268-02$

$3.9818-0$

2.6308
2.6908
2.7508

$2.750 \mathrm{~B}$
$2.0110 \mathrm{E}$

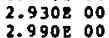

3.050800

3.110800
3.180800

3.180800

3.340800

3.420800
3.500800

3.580200

3.660E 00

3.820800

3.9 AnR $0 \mathrm{OA}$

4.060200
4.140800

4.220800

4.300800

4.3808
4.4608

8.540800

$4.630 \mathrm{R} 00$
4.730800

4.730800
4.830800

4.930200

5.130200

5.230800
5.330200

$5.430 \mathrm{O} 00$

$5.530 \mathrm{e}$.

5.730800

5.030200

3. $3.9618 \mathrm{~B}-03$

3. $6698-03$

$3.553 E-03$

$5.5048-02$

$5.1578-02$
$4.803 \mathrm{E}-02$

3. $3998-03$

3.3518-0

$3.3208-03$
$3.2078-03$

3.03JE-03

4.3718-02 3.0108-03

$0.2238-02 \quad 2.9343-03$

$3.7502-03$
3

$2.9538-02 \quad 2.7508-03$

$2.6158-02$
$2.7003-03$

$2.5958-02 \quad 2.530 \mathrm{~B}-03$

$2.685 \mathrm{E}-02 \quad 2.357 \mathrm{t}-03$

$2.6238-02 \quad 2.3798-03$

$2.6658-02 \quad 2.3138-03$

2.2158-03

$2.0018-02 \quad 2.233 \mathrm{E}-03$

$2.275 \mathrm{~B}-02 \quad 2.2018-03$

$\begin{array}{ll}2.116 \mathrm{P}-02 & 2.124 \mathrm{~B}-03 \\ 2.017 \mathrm{~B}-02 & 2.140 \mathrm{~B}-03\end{array}$

$\begin{array}{ll}2.017 \mathrm{~B}-02 & 2.140 \mathrm{~B}-03 \\ 1.9148-02 & 2.1968-03 \\ 1.6098-02 & 2.0868-03\end{array}$

$02 \quad 2.1458-03$

$1.609 \mathrm{R}-02$
$1.561 \mathrm{2}-02$

$1.584 \mathrm{E}-02 \quad 2.0008-03$

$1.7582-02$. $2.129 \mathrm{E}-03$

$1.008 \mathrm{E}-03$

$1.9198-02 \quad 1.928 \mathrm{~B}-03$

$1.8758-02 \quad 1.8908-03$

$\begin{array}{ll}1.7118-02 & 1.8698-03 \\ 1.5538-02 & 1.8138-03\end{array}$

6.0308 $00 \quad 1.5538-02 \quad 1.8138-03$

6.130800

$\begin{array}{rr}1.4968-02 & 1.8878-03 \\ 1.4758-02 & 1.6888-03\end{array}$

$\begin{array}{ll}1.4758-02 & 1.68888 \\ 1.4758-02 & 1.6738-03\end{array}$

$6.330 \mathrm{~g} 000 \quad 1.4758-25$

6.560800

$\begin{array}{ll}1.6278 \mathrm{z}-02 & 1.445 \mathrm{t}-03\end{array}$

$\begin{array}{ll}1.5262-02 \\ 1.3568-02 & 1.4278-03\end{array}$

6.800800

6.920800

7.040800

7.280800

7.400800
7.5208000
7.960800

7.6408 .00

7.760200
7.890200
0.970800

0.030800

8.170200

8. 310200

8.450800
8.590800

8.590800
8.730800

8. 870800

9. 150800

9.300800

9.620800

9.780800

1.0108 ol

1.026801

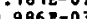

$1.3368-03$
$1.2518-03$

$8.500 \mathrm{z}-03$
$.1 .0958-03$

$8.1238-03 \quad 9.9228-04$
0.9280

$7.9468-03 \quad 9.3748-00$

$7.1618-03 \quad 0.3448-04$

$6.739 \mathrm{E}-03 \quad 7.937 \mathrm{~B}-04$

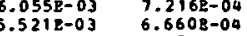

5.0562-03 $6.4518-04$

$5.8318-04$

$\begin{array}{ll}3.7108-03 & 5.9498-04\end{array}$

$\begin{array}{ll}2.0448-03 & 4.7958-94 \\ 2.1068-03 & 3.8158-04\end{array}$

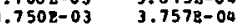

$1.6508-03$
1.5612

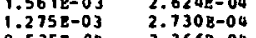

$8.5358-004 \quad 3.3662804$

.0528 of $2.7948-000$

$3.1018-04$
$1.2318-04$

$\begin{array}{ll}1.5908-04 & 1.2318-04 \\ 1.6978-00\end{array}$

I OTEGBLTEO DLTA

\begin{tabular}{|c|c|c|c|c|}
\hline oror & $\begin{array}{l}\text { BMRR } \\
\text { (O) }\end{array}$ & iv 181 & $\begin{array}{c}I-S 8 C \text { TIO } \\
(B / S B)\end{array}$ & $\begin{array}{l}\text { EQBOA } \\
(B / S B)\end{array}$ \\
\hline 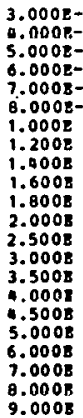 & $\begin{array}{l}-01= \\
-01= \\
01= \\
01= \\
01 \\
01= \\
-01= \\
00= \\
00= \\
00= \\
00= \\
00= \\
00= \\
00= \\
00= \\
00= \\
00= \\
000 \\
00= \\
00= \\
00= \\
00= \\
00=\end{array}$ & 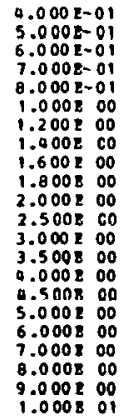 & $\begin{array}{l}1.7608-02 \\
1.3508-02 \\
9.2408-03 \\
3.065 \mathrm{z}-03 \\
6.532 \mathrm{z}-03 \\
3.493 \mathrm{E}-02 \\
2.4318-02 \\
1.3918-02 \\
1.499 \mathrm{z}-02 \\
1.1248-02 \\
1.2598-02 \\
3.1278-02 \\
2.6318-02 \\
2.298 \mathrm{z}-02 \\
1.5618-02 \\
1.3038-02 \\
1.0608-02 \\
1.7578-02 \\
1.5158-02 \\
8.7118-03 \\
5.9738-03 \\
1.9968-03\end{array}$ & 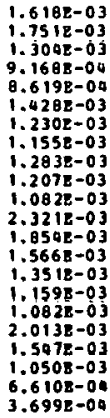 \\
\hline
\end{tabular}


DIPPEREATIAL CROSS SBCTIOHS POR GABM GAY PRODUCTION IN AM. THE PIBST SET

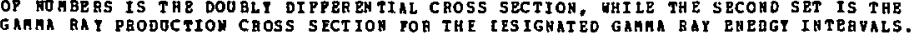

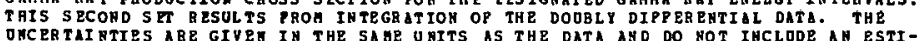

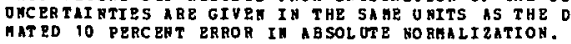

INCIDERT NEOTEON ENERGT $=11.98$ TO 13.95 MEV. ANGL $=125$ DEGREBS.

\begin{tabular}{|c|c|}
\hline $\begin{array}{c}10 \text { TON ERPRG } \\
\text { (IRP) }\end{array}$ & $\begin{array}{l}\text { X-SBCTION } \\
\text { (B/SR/ABV) }\end{array}$ \\
\hline 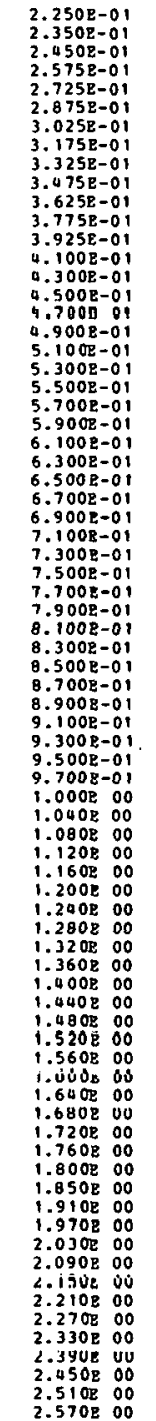 & 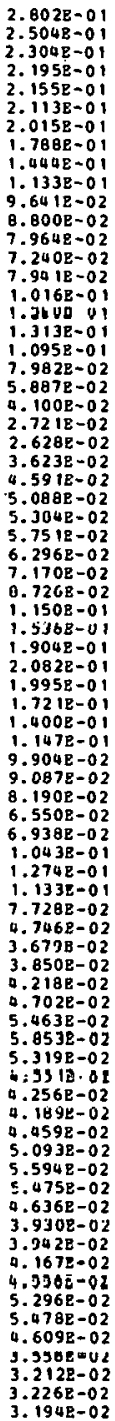 \\
\hline
\end{tabular}

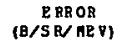

FHOTON EHREGT

$x-5$ Ection

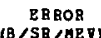

$3.274 \mathrm{E}-02$

2.630800
2.690800

$3.3738-02$

$3.7218-03$

(2.55

$2.251 \mathrm{E}-02$

$2.0672-0$

$2.010 \mathrm{E}-02$
$1.897 \mathrm{E}-02$

$1.824 \mathrm{E}-02$

$1.803 \mathrm{E}-02$

$1.828 \mathrm{E}-02$
$1.856 \mathrm{E}-02$

$1.908 \mathrm{E}-02$

$2.033 \mathrm{E}-02$

$2.057 \mathrm{~B}-02$

(i)

$1.904 \mathrm{E}-02$

$8578-02$

$1.206 \mathrm{E}-02$

$1.079 \mathrm{E}-\mathrm{C2}$
$1.066 \mathrm{~g}-02$

$1.066 \mathrm{~g}-02$
$1.039 \mathrm{~g}-02$

$1.039 \mathrm{~g}-02$
$1.138 \mathrm{E}-02$

$1.138 \mathrm{~B}-02$
$1.115 \mathrm{E}-02$

$1.032 \mathrm{E}-02$

2. 021803

9. $4132-03$

$9.033 \mathrm{E}-03$

$0.304 \mathrm{E}-03$
$8.005 \mathrm{~B}-03$

$8.0904-03$

$8.2012-03$
$8.298-03$

$8.122 \mathrm{P}-03$

8. $078 \mathrm{8}-03$
$7.756 \mathrm{~g}-03$

$7.7568-03$
$7.135 \mathrm{~g}-03$

$7.1358 \mathrm{~g}-03$
$6.741 \mathrm{~B}-03$

. $103 \mathrm{E}-0$

?.3918-03

7.012 $12-03$

6. $3528-03$

6. $205 \mathrm{E}-03$

$.946 \mathrm{E}-0 \mathrm{~J}$

5. $868 \mathrm{~B}-03$
$6.200 \mathrm{~g}-03$

6. $4028-03$

$6.003 \mathrm{~B}-03$
$6.307 \mathrm{z}-03$

6. $6258-03$

2.750800
2.8108
2.8000

2.8108 00

$2.870 R$
2.9300
2.900

3.050800

3.110800
3.180800

3. $260 \mathrm{e} 00$

3. 340800
3. 420800

3.500800

3. $500 \mathrm{Q} 00$

Tinn $\mathrm{n}$

3.820800
3.900200

3.9 $80 \mathrm{O}$ OO

$4.060 \mathrm{R}$
$4.140 \mathrm{O} O$

4.220800
4.300800

4.300800

4.3808
4.4608

540200

$4.630 \mathrm{~B} 00$
4.730800

4.830200

4.930200

5.030800
5.130800

$5.330 \mathrm{~B} 00$

5.430800
5.530200

5.530200
5.630800

5.7308 OO

5.930800

$6.030 \mathrm{P} 00$
$6.130 \mathrm{E} 00$

6.230200

6.330800

6.440800
6.560200

$6.600 \mathrm{~B} 00$

6.920800

7.040800

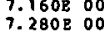

7.400800

1.6408 UU
7.7608

$6.0458-03$

$6.154 \mathrm{E}-03$

$5.524 \mathrm{R}-03$

$5.305 \mathrm{E}-03$

$5.5712-03$
$5.657 \mathrm{P}-0$.

r.vugy 0

$0.030 \mathrm{E}$
8.1700

8. 310200

8.450800

$8.590 \mathrm{R} 00$
$8.730 \mathrm{O} 00$

8.870800

9.010200
9.150200

$5.293 \mathrm{E}-0$

$.4612-0$

$4.520 \mathrm{R}-03$
$4.402 \mathrm{e}-03$

9.1508
9.3008 00

.8418-02 3.816E-0

$.174 \mathrm{E}-02$
$.1448-02$

$3.240 \mathrm{E}-02$

$2.8998-02$

$2.8488-02$

$3.0418-0$

$3.0848-02$
$3.1518-02$

$3.155 \mathrm{E}-02$

$2.8662-02$

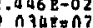

$1.8258-02$

$1.897 \mathrm{R}-02$

. $86 \mathrm{AE}-02$

$1.948 \mathrm{~g}-02$
$2.153 \mathrm{~g}-02$

$.179 \mathrm{E}-02$

$1.8288-02$
$1.3508-02$

$1.313 \mathrm{E}-02$

$1.4708-02$

$1.4508-07$

$1.019 \mathrm{E}-02$

$1008-02$

$1.2248-02$

1. $284 \mathrm{E}-02$

$1.1718-02$

1. $300 \mathrm{E}-02$

$1.4348-02$
$1.4188-02$

$1.299 \mathrm{e}-02$

$1.1258-02$
$9.2168-03$

a.281P-03

$1.003 \mathrm{E}-02$
$1.075 \mathrm{~B}-02$
1.0202

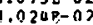

.. $024 \mathrm{~B}-03$

$6.8732-03$
$0.9058-03$

. $078 \mathrm{e}-03$

$4.4438=03$
$5.0428-03$

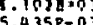

$5.4358-03$
$3.5718-03$
$3.0508-03$

$3.800 \mathrm{E}-03$
$3.084 \mathrm{E}-03$

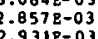

$2.9912-03$

$\begin{array}{ll}0 & 0\end{array}$

1.0018 .03

$9.620 \overline{\mathrm{E}} 00 \quad 1.067 \mathrm{8}-03$

9.7808 00

$4.539 \mathrm{~g}-03$
$4.246 \mathrm{E}-03$

$4.152 \mathrm{E}-03$

3. $019 \mathrm{gen}$

9.940200

$\because 1912-03$

$1.1148-03$
1.0698003

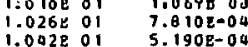

$695 \mathrm{~S}-03$
$709 \mathrm{E}-03$

$3.7008-03$

$3.394 \mathrm{~B}-03$
3. $30 \mathrm{Bg}-03$

$3,3558-03$

$3.0838-03$

$9678-03$

$2.980 \mathrm{~B}-03$
$2.934 \mathrm{Q}-03$

2.8268-03

$2.806 \mathrm{~B}-03$

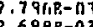

$2.6988-03$

$2.7128-03$

$2.61728-03$
$2.548-03$

2.5358-03

$2.4718-0.3$
$2.3978-0.3$
$2.4138-03$

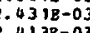

$3.366-0.0$

2. $368 \mathrm{E}-0 \mathrm{3}$

$2.278 \mathrm{~B}-03$

2. $1578-03$

$2.266 \mathrm{~B}-03$

$2.1848-03$
$2.136 \mathrm{~B}-03$

$2.113 \mathrm{~B}-0$

2.0828-03

$2.1022-03$

$2.019 \mathrm{E}-03$

$2.0478-03$
$2.0242-03$
1.03

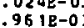

1.

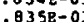

$1.7468-03$

$.679 \mathrm{~B}-03$
$1.609 \mathrm{~B}-03$

1. 53 a

1. $4538 \mathrm{E}-0.03$

. $3598-0$

1. $3114-03$

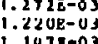

1. $1418-03$

$-1058=0$

$9.835 \mathrm{~B}-04$

$9.7968-04$

$9.7738-04$

8. $3028-04$

(3)

$6.231 \mathrm{P}-04$
G.
$6718-04 !$

7. $0538-00$

$4.368 \mathrm{ge}-04$
$4.767 \mathrm{~B}-04$

$4.7678-04$

.3210
$3.9798-04$

$\begin{array}{llll}3.8148-03 & 1.0428 & 5.1908-04 \\ 3.0902-03 & 1.058801 & 2.625 \mathrm{~B}-04\end{array}$

3. $1038-04$

IMTET DaTPD nat"

\begin{tabular}{|c|c|c|c|c|}
\hline IOTON E & $\begin{array}{r}\text { EHEBG } \\
\text { (ARE }\end{array}$ & (7) IK TERVAL & $\begin{array}{c}\text { x-SECTION } \\
(B / S R)\end{array}$ & $\begin{array}{l}8880 R \\
(B / 5 B)\end{array}$ \\
\hline 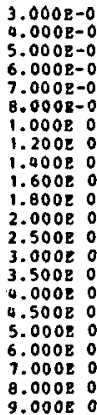 & $\begin{array}{l}01= \\
00= \\
00= \\
00= \\
00= \\
00= \\
00= \\
00= \\
00= \\
00= \\
00= \\
00= \\
00= \\
00= \\
00= \\
00= \\
00=\end{array}$ & 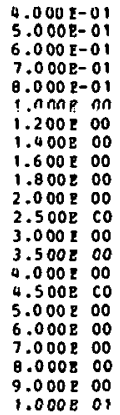 & 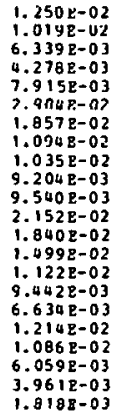 & 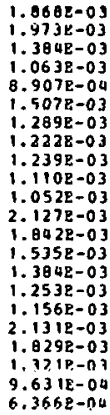 \\
\hline
\end{tabular}


DIPTERERTIAL CBOSS SECTIONS TOR GAHAA BAT PRODUCTION IM MY. THE PIRST SET OP WOABES IS THE DOU BLY DIPYER ENTIAL, CROSS SBCTION, YHILE THE SRCOHD SBT IS THE

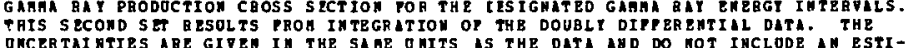

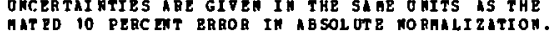

INCIDENT NBUTRON EMERGI = 13.95 TO $16.95 \mathrm{MEV}$. ANGLE = 125 DBGRZES.

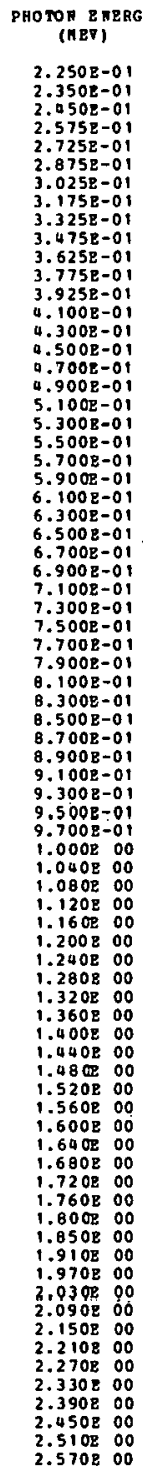

\begin{tabular}{|c|c|}
\hline $\begin{array}{l}\text { - SECTION } \\
B / S R / A B D)\end{array}$ & $\begin{array}{c}\text { EROOR } \\
\text { (B/SR ARV) }\end{array}$ \\
\hline 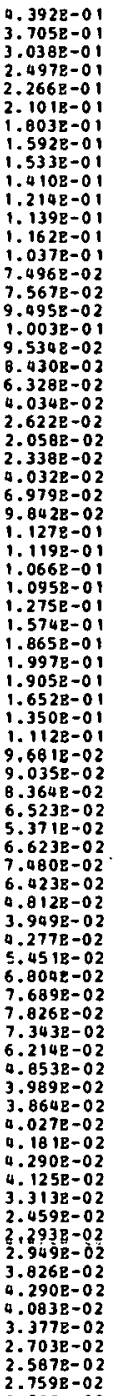 & 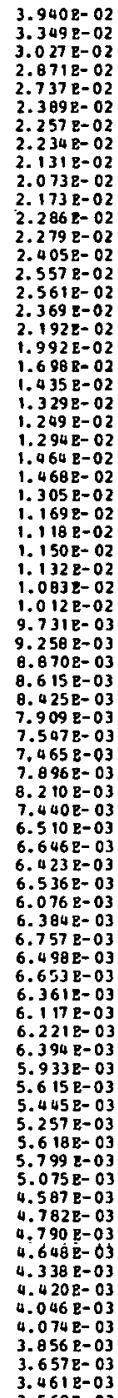 \\
\hline
\end{tabular}

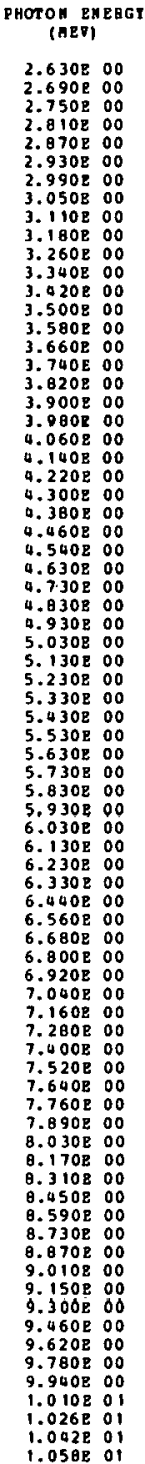

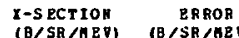

$2.4028-02 \quad 3.748 \mathrm{E}-03$ $2.5968-02 \quad 3.420 \mathrm{E}-03$ $2.785 \mathrm{~B}-02$
$2.557 \mathrm{~B}-02$ $3.578-02 \quad 3.2578-03$ $2.2858=02 \quad 3.1828-03$ $\begin{array}{ll}2.2898-02 & 3.04 E E-03 \\ 2.193 \mathrm{E}-02 & 3.144 \mathrm{E}-03 \\ 1.837 \mathrm{E}-02 & 3.1448-03\end{array}$ $\begin{array}{ll}1.8378-02 & 3.1448-03 \\ 1.5768-02 & 3.1418-03\end{array}$ $1.576 \mathrm{E}-02$
$1.714 \mathrm{E}-02$
$2.9918-03$ $2.0518-02 \quad 2.861 \mathrm{E}-03$ $1.8568-020158-03$ $1.736 \mathrm{E}-02 \quad 2.633 \mathrm{E}-03$ $1.577 \mathrm{E}-02 \quad 2.6068-03$ $1.253 \mathrm{E}-02 \quad 2.558 \mathrm{E}-03$ $2.5038-03$ (18E-0 $1.0918-02 \quad 2.6768-03$ $.323 \mathrm{E}-02-3.359 \mathrm{E}-03$ $1.4848-02 \quad 2.2978-03$ $1.481 \mathrm{R}-02 \quad 2.211 \mathrm{E}-03$ 1.3618-02 $2.3038-03$ $1.069 \mathrm{E}-02 \quad 2.2 .16 \mathrm{E-0}$ $9.9298-03 \quad 2.2578-0$ $6.000 \mathrm{E}-03 \quad 2.167 \mathrm{~B}-03$ $6.335 \mathrm{R}-03 \quad 2.140 \mathrm{~B}-0$ $6.6918-03 \quad 2.187 \mathrm{E}-0$ $5.2822-03 \quad 2.0902-03$ $3.910 \mathrm{z}-03 \mathrm{~B}$ $5.3118-03 \quad 2.0008-03$ $6.7028-03 \quad 2.0338-03$ $1.976 \mathrm{~B}-03$
$1.931 \mathrm{~B}-03$ $8.216 \mathrm{E}-03 \quad 1.900 \mathrm{E}-03$ $6.921 \mathrm{z}-03 \quad 1.885 \mathrm{~B}-03$ $5.3828-03 \quad 1.8538-03$ 1.8728-0 $1888-03 \quad 1.8028-03$ $7.7678-03 \quad 1.7158-03$ $6.637 \mathrm{E}-03 \quad 1.653 \mathrm{E}-0$

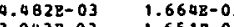
$3.0438-03 \quad 1.6518-03$ $4.5528-03 \quad 1.4828-03$ $5.625 \mathrm{E}-03$ $6.2208-03 \quad 1.350 \mathrm{z}-03$ $5.726 \mathrm{E}-03 \quad 1.200 \mathrm{E}-03$ $4.352 \mathrm{R}-03 \quad 1.1918-0$ $2.9738 \mathrm{E}-1.130 \mathrm{e}-0 \mathrm{~g}$ 1. $.0218-03 \quad 1.0298-03$ $3.5198-03 \quad 1.006 \mathrm{E}-03$ $3.101 \mathrm{R}-03 \quad 9.839 \mathrm{E}-0$ $2.269 \mathrm{E}-03 \quad 9.647 \mathrm{E}-09$ $1.795 \mathrm{z}-03 \quad 9.080 \mathrm{E}-0$ 1.57203 $1.177 \mathrm{E}-03$ a $114 \mathrm{R}-00$ $1.2418-03 \quad 7.2968-04$ $1.3808-03 \quad 6.279 \mathrm{R}-04$ $1.6618-03 \quad 6.4528-04$ $1.715 \mathrm{E}-03 \quad 6.052 \mathrm{E}-00$ (.). $5.198 \mathrm{E}-04 \quad .797 \mathrm{E}-04$ $3.647 \mathrm{E}-04 \quad 3.3518-00$ $2.926 \mathrm{E}-04 \quad 3.616 \mathrm{E}-04$ $3.129 \mathrm{E}-04 \quad 4.2318-04$ $\begin{array}{ll}3.498 \mathrm{E}-04 & 2.670 \mathrm{R}-04 \\ 3.910 \mathrm{E}-00 & 1.688 \mathrm{E}-00\end{array}$ $2.911 \mathrm{R}-04 \quad 2.264 \mathrm{E}-04$

I HTEG RATBD OATA

\begin{tabular}{|c|c|c|c|c|}
\hline OPOH & $\begin{array}{l}\text { BY घAC } \\
\text { (AR }\end{array}$ & II IN & $\begin{array}{c}2-\text { EBCTI ON } \\
\text { (B/SR) }\end{array}$ & $\begin{array}{l}\text { 8BROR } \\
\text { (B/SB) }\end{array}$ \\
\hline $\begin{array}{l}3.0008-0 \\
9.0008-0 \\
5.0008-0 \\
6.00008-0 \\
7.0008-0 \\
8.0008-0 \\
1.0008 \\
1.2008 \\
1.0008 \\
1.6008 \\
1.0008 \\
2.0008 \\
2.5008 \\
3.0008 \\
3.5008 \\
4.0008 \\
9.5008 \\
5.0008 \\
6.0008 \\
7.0008 \\
8.0008 \\
9.0008\end{array}$ & $\begin{array}{l}01= \\
01 \\
01 \\
00 \\
00 \\
00 \\
00 \\
00 \\
00 \\
00 \\
00 \\
00 \\
00\end{array}=$ & 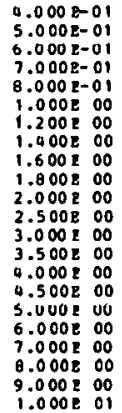 & 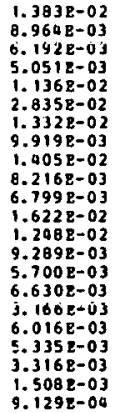 & $\begin{array}{l}2.205 E-03 \\
2.415 E-03 \\
1.3428-03 \\
1.3428-03 \\
1.1008-03 \\
1.678 z-03 \\
1.3708-03 \\
1.2908-03 \\
1.256 E-03 \\
1.1118-03 \\
9.798 z-04 \\
2.1008-03 \\
1.6068-03 \\
1.4678-03 \\
1.2978-03 \\
1.1418-03 \\
1.0818-03 \\
1.9478-03 \\
1.5638-03 \\
1.0878-03 \\
9.0328-00 \\
9.6558-04\end{array}$ \\
\hline
\end{tabular}




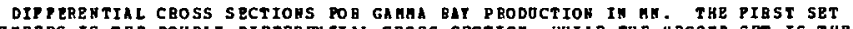

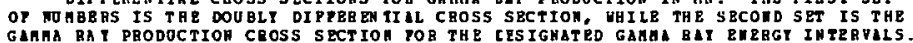
TAIS SECOHD SET RESULTS PROA IHTR GR ATION OP THE DOOBLP DIPPER EHTIAL DATA. THE

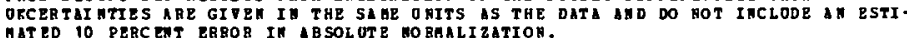

IKCTDENT HEUTROA EHERGT $=16.95$ TO 19.95 HEV, ANGLE $=125$ DEGREES.

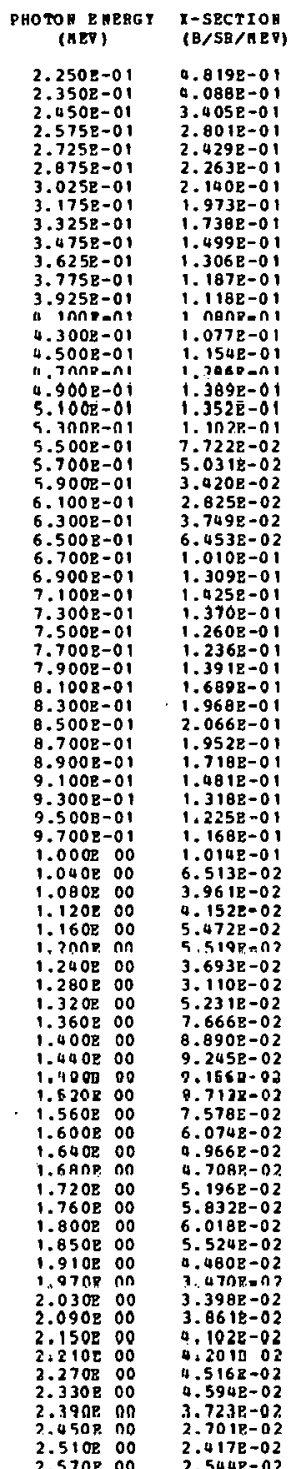

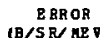

EHOTON EREBGI T-SECTION EREOB

$5.628 \mathrm{~g}-02$

$4.7878-02$
$4.532 \mathrm{~B}-02$

$4.317 \mathrm{E}-02$

$3.760 \mathrm{R}-02$

$3.321 \mathrm{R}-02$

$2.9738-02$
$2.919 \mathrm{E}-02$

$2.890 \mathrm{E}-02$

$2.829 \mathrm{E}-02$

$2.804 \mathrm{E}-02$
$3.128 \mathrm{E}-02$

3. $296 \mathrm{E}-02$

$3.669 \mathrm{E}-02$
$3.398 \mathrm{E}-02$

2. $959 \mathrm{E}-02$

$2.6708-02$

$2.042 \mathrm{z}-02$

$1.759 \mathrm{z}-02$
$1.732 \mathrm{z}-02$

$1.768 \mathrm{~B}-02$

$1.8618-02$
$1.838 \mathrm{E}-02$

$1.717 \mathrm{~B}-02$

$1.617 \mathrm{E}-02$
$1.535 \mathrm{z}-02$
$1.527 \mathrm{e}-02$

1. 518 R-02

$1.478 \mathrm{E}-02$

$1.436 \mathrm{~B}-02$
$1.309 \mathrm{E}-02$

1. $3318-02$

1. $302 \mathrm{E}-02$

1. $1918-02$

$1.109 \mathrm{P}-02$
$1.050 \mathrm{R}-02$

$1.050 \mathrm{~B}-02$
$1.083 \mathrm{~B}-02$

1. $101 \mathrm{E}-02$

$1.036 \mathrm{E}-02$
$\mathrm{~B} .900 \mathrm{~B}-03$

8. $900 \mathrm{~B}-0 \mathrm{~J}$
$8.860 \mathrm{z}-03$

8. $4848-03$

B. $699 \mathrm{~B}-03$

$9.001 \mathrm{E}-03$

$8.857 \mathrm{p}-03$

$9.229 \mathrm{E}-03$
$9.749 \mathrm{z}-03$

$9.7498-03$
$8.9638-03$

$0.11818 \cdot 03$

8. $4412-03$

7. $949 \mathrm{z}-03$

$7.522 \mathrm{~g}-03$

7. $4528-03$

$7.367 \mathrm{~g}-03$

6.827 $\mathrm{g}-03$

6. $2478-03$

2.630800

$2.643 \mathrm{~B}-02$

$\left(2 B / A B D_{1}\right.$

$2.750 \mathrm{R} 00 \quad 2.865 \mathrm{E}-02 \quad 4.4 \mathrm{CBR}-03$

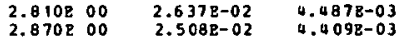

$2.930800 \quad 2.704 \mathrm{~B}-02 \quad 4.152 \mathrm{~B}-03$

$2.990 \mathrm{~B} 00 \quad 2.797 \mathrm{P}-02 \quad 4.180 \mathrm{~B}-03$

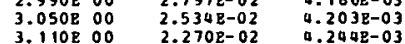

$\begin{array}{lll}3.180800 & 2.308 \mathrm{~B}-02 & 4.169 \mathrm{~g}-03\end{array}$

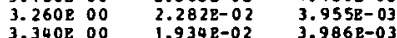

$3.420800 \quad 1.8828-02 \quad 9.0058-03$

$3.580200 \quad 2.1548002$

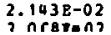

$3.820 \mathrm{E} 00$

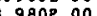

$4.060 \mathrm{R} 00$

$1.638 \mathrm{~B}-02$
$1.098 \mathrm{~B}-02$

ก..372R-0.3

140800
$1.0268-02$
$3.2908-03$

$\begin{array}{llr}.220 \mathrm{E} 00 & 1.229 \mathrm{E}-02 & -3.067 \mathrm{~B}-03 \\ 1.231 \mathrm{E}-02 & 3.000 \mathrm{~B}-03\end{array}$

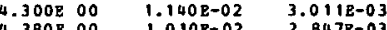

.

$4.540800 \quad 7.098 \mathrm{P}-03 \quad 2.735 \mathrm{~B}-03$

$\begin{array}{ll}4.730 \mathrm{~B} & 00 . \\ 4.830 \mathrm{~B} & 00 \\ 5.0308 & \end{array}$

4.930800

5.030800
5.130800
5.2308

$5.330 \mathrm{E} 000$

$\begin{array}{ll}5.4308 & 00 \\ 5.5308 & 00\end{array}$

5.630800

5.830800
5.9308

$6.030 \mathrm{~B} 00$

6.1300
6.300

6. $330 \mathrm{R} 00$

6.440800
$6.560 \mathrm{E}$
6.60

6.800000

6.920800
7.040800

$7.160 \mathrm{R}$ oO

7.2808000
7.400800

$7,4428-03$

$7.619 \mathrm{R}-03$

$6.933 \mathrm{R}-03$
$7.690 \mathrm{R}-0.03$

$2.5868-03$

$2.5268-03$
$288-03$

$2.6148-03 \quad 2.4598-03$

$2.3318-03$

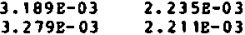

3.5848-03 2. $297 \mathrm{~B}-03$

$\begin{array}{ll}4.3048-03 & 2.2178-03 \\ 4.603 \mathrm{z}-03 & 2.182 \mathrm{~B}-03 \\ 6.03 & \end{array}$

$5.3938-03 \quad 2.1178-03$

$6.3978-03 \quad 2.0818-03$

$4.609 \mathrm{z}-03 \quad 2.051 \mathrm{~B}-03$

1. $834 \mathrm{~B}-03$

$9.2168-04 \quad 1.8258-03$

$3.994 \mathrm{E}-03$

$5.889 \mathrm{z}-03 \quad 1.573 \mathrm{E}-03$

$5.343 \mathrm{~B}-03 \quad 1.455 \mathrm{~B}-03$

$2.605 \mathrm{~B}-03$

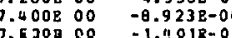

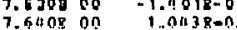

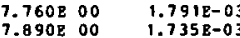

อ. $030800 \quad 1.2358-03$

0
0

8. 310200

8.590800

8.730200
8.8700 00
9.1508

$9 . n \operatorname{lng}$ an

9.150800

5. $936 \mathrm{z}-03$

$6.1018-03$

$9,460 \mathrm{O} 00$

9.780000
9.9408000
.9

5. $9312-03$

$5.4642-03$
$5.3518-0.3$
$5.3632-03$

9.9408
1.0108

1.0268 ? 1.0428

$6.407 \mathrm{R}-04$

6. $9430 \mathrm{p}$

$1.0878-03$

$7.6538-04$

$-1.0862-04$

$-4.433 \mathrm{~B}-05$

$2.5302-04$
$5.7562=01$

$5.0228-04$
$5.0578-04$

$3.8018-04$

5. $157 \mathrm{P}-03$

1.9862-04

?. $307 \mathrm{P}-03$

$1.200 \mathrm{E}-03$

$1.1027-97$

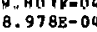

$9.2298-04$

$8.4258-04$

8.0268-04

$8.0538-04$
$7.4518-04$

$6.6368-09$

$6.327 \mathrm{R}-04$
$5.779 \mathrm{~g}-04$
$6.66218-04$

5. $1628 \mathrm{R}-04$

$4.6618-04$

$4.0498-04$
$4.1558-04$

3. $8638 \mathrm{~B}-04$

$3.2 \mathrm{AP}-0 \mathrm{Q}$
$3.6678-04$

$3.3198-84$

$1.0612-04$
$2.0038-04$

I RTEG RATED OATA

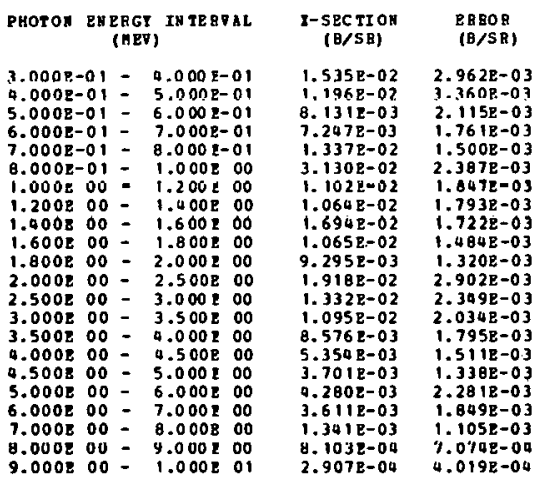




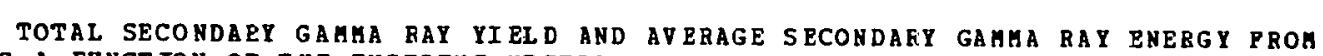
MN AS A PONCT TON OF THE INCIDENT NE OTRON ENERGT. THESE DATA RESULT PBOM A PULSE HEIGHT HEIGHTING AUALYSIS POR PULSE HEIGHTS GREATER THAN 0.620 HEV. UNCERTAINTIES ARE GIVEN IN RARENTHIESES IN THE SAHE UNITS AS THE DATA. THE UNCERTAINTIES IN TOTAL YIELD DO BOT INCLODE A 10 PERCENT ERROR IN ABSOLOTE NORAALIZATION. THE ANGLE IS 125 DEGREES.

\begin{tabular}{|c|c|c|c|}
\hline T. & $\begin{array}{l}\text { NERG } \\
\text { PREA D } \\
\text { (GEV) }\end{array}$ & $\begin{array}{l}\text { BCONDARY } \\
\text { YIELD }\end{array}$ & $\begin{array}{l}\text { Y PHOTON } \\
\text { (B/SR) }\end{array}$ \\
\hline $\begin{array}{l}0.150 \\
0.251 \\
0.351 \\
0.451 \\
0.551 \\
0.650 \\
0.747 \\
0.845 \\
0.948 \\
1.127 \\
1.375 \\
1.621 \\
1.878 \\
2.126 \\
2.379 \\
2.636 \\
2.870 \\
3.112 \\
3.371 \\
3.631 \\
3.886 \\
4.129 \\
4.376 \\
4.621 \\
4.864\end{array}$ & $\begin{array}{l}0.101 \\
0.100 \\
0.100 \\
0.101 \\
0.099 \\
0.098 \\
0.096 \\
0.102 \\
0.103 \\
0.256 \\
0.240 \\
0.250 \\
0.264 \\
0.232 \\
0.274 \\
0.240 \\
0.227 \\
0.257 \\
0.261 \\
0.259 \\
0.251 \\
0.236 \\
0.257\end{array}$ & 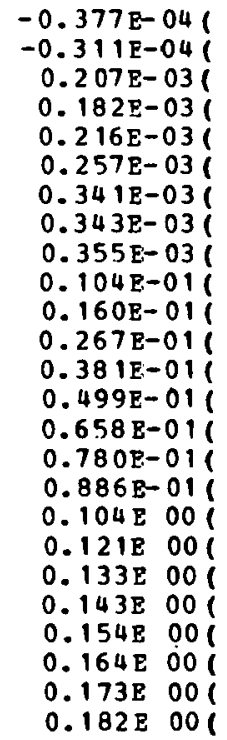 & 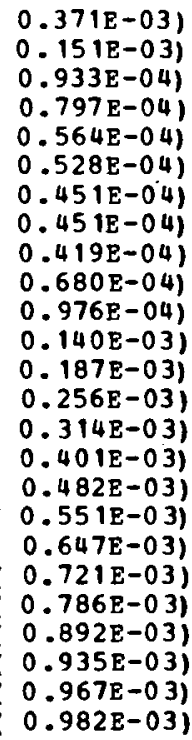 \\
\hline
\end{tabular}

AV ERA GE EN ERGY

$$
\text { ( } \mathrm{BEV}
$$

$-0.275 \mathrm{E} \quad 02(0.272 \mathrm{E} \quad 03)$ $0.142 \mathrm{E} 02(0.696 \mathrm{E} \quad 02)$ $0.504 \mathrm{E} 01(0.251 \mathrm{E} 01)$ $0.484 \mathrm{E} 01(0.238 \mathrm{E} 01)$ $0.431 \mathrm{E} 0110.130 \mathrm{E} 011$ $0.466 \mathrm{E} \mathrm{01}(0.109 \mathrm{E} 01)$ $0.420 \mathrm{E} 01(0.652 \mathrm{E} 00)$ $0.424 \mathrm{E} 01(0.645 \mathrm{E} 00)$ $0.380 \mathrm{E} 01(0.528 \mathrm{E} 00)$ $0.961 \mathrm{E} 00(0.105 \mathrm{E}-01)$ $0.946 \mathrm{E} 00(0.863 \mathrm{E}-02)$ $0.103 \mathrm{E} 01(0.749 \mathrm{~B}-02)$ $0.109 \mathrm{E} 01(0.726 \mathrm{E}-02)$ $0.121 \mathrm{E} 01(0.833 \mathrm{E}-02)$ $0.132 \mathrm{~B} 01(0.84 \mathrm{~B}-02)$ $0.132 \mathrm{E}$ OI( $0.84 \mathrm{E}-02)$ - 150 or o - TsOe OT( O. 108E-0

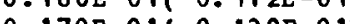
$0.170 \mathrm{E}$ OTR $0.120 \mathrm{E}-01)$ $0.176 \mathrm{E}$ OI( $0.126 \mathrm{E}-0 \mathrm{~T})$ $0.182 \mathrm{E}$ O1 $0.132 \mathrm{E}-01)$ $0.186 \mathrm{E}$ OI 0.14 1E-O $0.191 \mathrm{E} 01(0.142 \mathrm{E}-01)$ $0.195 \mathrm{E} 01(0.145 \mathrm{E}-01)$ $0.199 E 01(0.141 E-01)$

$\begin{array}{cc}\text { INC.NT. } & \text { EN ERGY } \\ \text { ENERGY } & \text { SPREAD } \\ \text { (HEV) } & \text { (HEV) } \\ & \\ 5.243 & 0.506 \\ 5.755 & 0.518 \\ 6.272 & 0.516 \\ 6.778 & 0.497 \\ 7.256 & 0.459 \\ 7.739 & 0.506 \\ 8.271 & 0.559 \\ 8.765 & 0.427 \\ 9.243 & 0.529 \\ 9.758 & 0.502 \\ 10.241 & 0.463 \\ 10.721 & 0.496 \\ 11.235 & 0.533 \\ 11.739 & 0.474 \\ 12.228 & 0.504 \\ 12.749 & 0.537 \\ 13.245 & 0.455 \\ 13.712 & 0.480 \\ 14.205 & 0.506 \\ 14.725 & 0.534 \\ 15.497 & 1.010 \\ 16.477 & 0.950 \\ 17.471 & 1.038 \\ 18.460 & 0.940 \\ 19.438 & 1.016\end{array}$

SECONDART PHOTON YIELD (B/SR)

DVERAGE RNERG (HEV)

$0.194 \mathrm{E} 00(0.967 \mathrm{E}-03)$ $0.208 \mathrm{E} 00(0.104 \mathrm{E}-02)$ $0.216 \mathrm{E} 00(0.106 \mathrm{E}-02)$ $0.234 \mathrm{E} 00(-0.114 \mathrm{E}-\mathrm{C} 2)$ $0.246 \mathrm{E} 00(0.128 \mathrm{E}-02)$ $0.242 \mathrm{E} 00(0.125 \mathrm{E}-02)$ $0.261 \mathrm{E} 00(0.130 \mathrm{E}-02)$ $0.277 \mathrm{E} 00(0.150 \mathrm{E}-02)$ $0.260 \mathrm{E} 00(0.145 \mathrm{E}-02)$ $0.283 \mathrm{E} 00(0.162 \mathrm{E}-02)$ $0.294 \mathrm{E} 00(0.182 \mathrm{E}-02)$ $0.287 \mathrm{~B} 00(0.1898-02)$ $0.283 \mathrm{~B}$ 00( $0.196 \mathrm{~B}-02)$ $0.268 \mathrm{~B} 00(0.215 \mathrm{~B}-02)$ $0.268 \mathrm{E} 00(0.215 \mathrm{E}-02)$ $0.235 \mathrm{E}$ 00( $0.210 \mathrm{E}-02)$ $0.217 \mathrm{E}$ OO $0.224 \mathrm{E}-02)$ $0.186 \mathrm{~B}$ 00 $0.260 \mathrm{E}-02)$ $0.173 \mathrm{0O}(0.266 \mathrm{E}-02)$ $0.173 \mathrm{E} 00(0.266 \mathrm{E}-02)$ $0.169 \mathrm{E} 00(.0 .304 \mathrm{E}-02)$ $0.161 \mathrm{E} 00(0.258 \mathrm{E}-02)$ $0.158 \mathrm{E}$ 00( $0.289 \mathrm{E}-02)$ $0.166 \mathrm{E} 00(0.313 \mathrm{E}-02)$ $0.182 \mathrm{E} 00(0.357 \mathrm{E}-02)$ $0.194 \mathrm{E} 00(0.399 \mathrm{E}-02)$
$0.204 \mathrm{E} 01(0.1318-01)$ $0.211 E 0110.135 \mathrm{E}-0$ 11 $0.221 \mathrm{E} 0110.139 \mathrm{E}-011$ $0.229 \mathrm{E} 01(0.142 \mathrm{E}-01)$ $0.239 \mathrm{E} 01(0.156 \mathrm{E}-01)$ $0.248 \mathrm{E} 01(0.160 \mathrm{E}-0)$ $0.257 \mathrm{E} \mathrm{O1}(0.162 \mathrm{E}-01)$ $0.266 \mathrm{E} 01(0.183 \mathrm{E}-0)$ $0.273801(0.191 \mathrm{E}-01)$ $0.281 \mathrm{E} 01(0.203 \mathrm{E}-01)$ $0.287 \mathrm{E}$ C1 $0.224 \mathrm{E}-011$ $0.2 \mathrm{G3E} 01(0.242 \mathrm{E}-011$ $0.256 \mathrm{E} 0110.257 \mathrm{~B}-011$ $0.295 \mathrm{~B} 01(0.298 \mathrm{~B}-011$ $0.286 \mathrm{O} 01(0.3248-011$ $0.285 \mathrm{E} 01(0.366 \mathrm{~B}-01)$ $0.277 \mathrm{E} 01(0.4418=01)$ $0.263 \mathrm{E} 0110.4638-011$ $0.253 \mathrm{~B} 01(0.498 \mathrm{~B}-011$ $0.243 \mathrm{E} 01(0.543 \mathrm{E}-01)$ $0.227 \mathrm{E} 01(0.452 \mathrm{E}-01)$ $0.210 \mathrm{E}$ OI ( $0.4858-01)$ 0.2 CBE OI $0.500 E-011$ 0.2 CBE OI $0.530 \mathrm{E}-01)$ $0.213 \mathrm{E} 01(0.562 \mathrm{E}-01)$ 
TOTAL SECONJABY GAHA RAY YIELD AND A ARRAGE SECONDARY GaHHA RAT EMERGY $F$ ROM MN AS A PUNCTIOS O? THE INCIDERT NEUT RCN BNERGY. THESE DATA RESULT FRC. HEIGHT AEIGHTING ANALYSIS FOR PUL SE HEIGHTS GREATER THAN 0.220 HEV. [NCERTAINTIES ARE GIVZN IN PARENTHIESES IN THE SAMP ONITS AS THE DATA. THE ONCERTAIHTIES IN TOTAL YIE-D LO JOT INCLUDE A. 10 PERCENT ERROR IN ABSOLOTE NORGALIZATION. THE ANGLE IS 125 DEGREZS.

\begin{tabular}{|c|c|}
\hline $\begin{array}{l}N C, N T \text {. } \\
\text { NE RGY } \\
\text { (IEVV) }\end{array}$ & $\begin{array}{l}\text { ENE RG Y } \\
\text { SPREA D } \\
\text { (HEV) }\end{array}$ \\
\hline $\begin{array}{l}0.150 \\
0.251 \\
0.351 \\
0.451 \\
0.551 \\
0.650 \\
0.747 \\
0.845 \\
0.948 \\
1.127 \\
1.375 \\
1.621 \\
1.878 \\
2.126 \\
2.379 \\
2.636 \\
2.870 \\
3.112 \\
3.371 \\
3.631 \\
3.886 \\
4.129 \\
4.376 \\
4.621 \\
4.864\end{array}$ & $\begin{array}{l}0.101 \\
0.100 \\
0.100 \\
0.101 \\
0.099 \\
0.098 \\
0.095 \\
0.102 \\
0.103 \\
0.256 \\
0.240 \\
0.250 \\
0.264 \\
0.232 \\
0.274 \\
0.240 \\
0.227 \\
0.257 \\
0.261 \\
0.259 \\
0.251 \\
0.236 \\
0.257 \\
0.233 \\
0.252\end{array}$ \\
\hline
\end{tabular}

SECONDARY PHOTON TIEL: (B/SR)

$0.130 \mathrm{E}-03(0.6022-03)$ $0.515 \mathrm{E}-04(0.229 \mathrm{E}-03)$ $0.277 \mathrm{E}-0 \mathrm{3}(0.143 \mathrm{E}-03)$ $0.360 \mathrm{E}-03(\mathrm{C} .116 \mathrm{~F}-03)$ $0.348 \mathrm{E}-03(0.825 \mathrm{~s}-04)$ $0.519 \mathrm{E}-03(0.778 \mathrm{E}-04)$ $0.533 \mathrm{E}-03(0.815 \mathrm{E}-0.41$ $0.616 \mathrm{E}-03(0.978 \mathrm{E}-04)$ $0.628 \mathrm{E}-03(0.106 \mathrm{E}-03)$ $0.119 \mathrm{E}-01(0.163 \mathrm{E}-03)$ $0.185 \mathrm{E}-01(0.213 \mathrm{r}-031$ $0.314 \mathrm{E}-01(0.267 \mathrm{E}-031$ $0.449 \mathrm{E}-0110.328 \mathrm{E}-031$ $0.495 \mathrm{E}-01(0.400 \mathrm{E}-03 \mathrm{~B}$ $0.78 \mathrm{E}-01 \mathrm{l} 0.400 \mathrm{E}-03$ $0.789-0110.475 E-03$ $0.934 \mathrm{E}-01(0.584 \mathrm{E}-03)$ . $0.125 \mathrm{E}$ OO $0.763 \mathrm{E}-03$ $0.146 \mathrm{E} 00(0.903 \mathrm{E}-03$ 0.161 E 00 ( $0.100 E-02$. $0.174 \mathrm{E}$ 00 (O. TO9E-02) $0.186 \mathrm{E}$ 0O C. 120E-02. C. $200 \mathrm{E}$ 00( $\mathrm{C} .125 \mathrm{E}-02$ ) $0.210 \mathrm{E} 00$ ( C. 133E-02 $0.221 E$ OC $C .136 E-02$ :
AV ERAGE EN ER GY

$$
\text { (AEV) }
$$

$\begin{array}{llllll}0.835 \mathrm{E} & 011 & 0.391 \mathrm{E} & 0.2\end{array}$ $0.930 \mathrm{E}$ O1 $0.419 \mathrm{E} 02)$ $0.382 \mathrm{E} 01(0.212 \mathrm{E} 01)$ $0.260 \mathrm{E} 01(0.99 \mathrm{CE} 00)$ $0.278 \mathrm{E} 01(0.770 \mathrm{E}$ OJ) $0.247 \mathrm{E}$ 01( $0.447 \mathrm{E}$ OD) $0.280 \mathrm{E} 01(0.481 \mathrm{E} 0 \mathrm{~J})$ $0.253 \mathrm{E} 01(0.442 \mathrm{E} 0.0)$ $0.228 \mathrm{E} 01(0.419 \mathrm{E} 00)$ $0.883 \mathrm{E} 00(0.145 \mathrm{E}-01)$ $0.865 E 00(0.117 E-01)$ 0.9288 00( $0.9148-02)$ $0.974800(0.9298-02)$ 0.1078 O1( $0.8408-02)$ $116 \mathrm{E} 01(0.331 \mathrm{E}-02)$ 0.127 Q $0.139 \mathrm{E}$ O - 148E OT( $0.112 \mathrm{E}-01)$ . 152E OT( O. 11EE-O . 156E OI( O. ILEE-O?) $0.160 \mathrm{E}$ OI( $0.127 E-01)$ $0.163 \mathrm{E}$ O1( $0.126 \mathrm{E}-01)$ $0.167 \mathrm{E} 01(0.130 \mathrm{E}-0$ !) $0.170 \mathrm{E} 01(0.128 \mathrm{E}-0 \mathrm{I})$

\section{BNERGY SPREAD (HEV) (IEV) \\ SECONDARY PHOTON Y IELD $(B / S B)$}

$\begin{array}{lll}5.243 & 0.506\end{array}$ 5.7550 .518 6.2720 .516 $6.778 \quad 0.497$ $7.256 \quad 0.459$ 7.7390 .506 8.2710 .559 $8.765 \quad 0.427$ 9.2430 .529 $9.758 \quad 0.502$ 10.2410 .463 10.7210 .496 $11.235 \quad 0.533$ 11.7390 .434 12.2280 .574 $12.749 \quad 0.537$ 13.2450 .537 13.2450 .455 13.7120 .480 14.7250 .534 15.7250 .534 $15.497 \%$ 16.4770 .950 17.4711 .038 18.4600 .940 $19.438 \quad 1.016$
$0.235 \mathrm{E} \mathrm{OOC} 0.132 \mathrm{E}-02)$ $0.253 \mathrm{E} 00(0.139 \mathrm{E}-02)$ $0.263 \mathrm{E} 00(0.145 \mathrm{E}-02)$ $0.283 \mathrm{E} 00(0.154 \mathrm{E}-02)$ $0.299 E$ 00( $0.164 E-02)$ $0.294 \mathrm{E} \mathrm{DO}(0.162 \mathrm{E}-02)$ $0.315 \mathrm{E} \mathrm{DO}(0.177 \mathrm{E}-02)$ $0.336 \mathrm{E} 00(0.202 \mathrm{E}-02)$ $0.314 \mathrm{E} 00 .(0.190 \mathrm{E}-02)$ $0.337 \mathrm{E}$ O0( $0.220 \mathrm{E}-02)$ $0.355 \mathrm{E} 00(0.240 \mathrm{E}-02)$ $0.350 \mathrm{E} 00(0.242 \mathrm{E}-02)$ $0.340 \mathrm{~B} \mathrm{O0}(0.256 \mathrm{P}-02)$ $0.321 \mathrm{E} 30(0.288 \mathrm{~B}-02)$ $0.321 \mathrm{~B}$ ग0 $0.288 \mathrm{E}-02)$ $0.265 \mathrm{~B}$ 00 $0.29 \mathrm{E}-02$ $0.246 \mathrm{~B}$ DO $0.33 \mathrm{E}-02$ $0.233 \mathrm{~B}$ D $0.355 \mathrm{E}-02)$ 0.23 B DO $0.35 \mathrm{~B}-\mathrm{C2}$ $0.219 \mathrm{E}$ 00 $0.380 \mathrm{E}-02$ $0.215=$ DO $0.350 \mathrm{E}-021$ 0.212 D $010.377 \mathrm{~B}-02)$ 0.212 o $0.378-02$ $0.221 \mathrm{E}$ ग( $0.431 \mathrm{E}-02)$ $0.257 \mathrm{E} 20(0.583 \mathrm{E}-02)$

\section{AV ERAGE ENERGT} (AEV)

$0.175801(0.1188-01)$ $0.180 \mathrm{E} 01(0.120 \mathrm{E}-01)$ $0.188 \mathrm{E} 01(0.125 \mathrm{E}-0)$ $0.195 \mathrm{E} 01(0.127 \mathrm{E}-0)$ $0.204 E 01(0.135 E-01)$ $0.211 \mathrm{E} 01(0.140 \mathrm{E}-01)$ $0.219 \mathrm{E} 01(0.147 \mathrm{~B}-01)$ $0.226 \mathrm{E} 01(0.163 \mathrm{E}-0.1)$ $0.232 E 01(0.168 E-01)$ $0.241 E 01(0.187 E-01)$ $0.244 \mathrm{E} 01(0.198 \mathrm{E}-01)$ $0.247 \mathrm{E} 0110.207 \mathrm{E}-011$ $0.253 \mathrm{E} 0110.228 \mathrm{~B}-011$ $0.253 \mathrm{E} 01(0.271 \mathrm{~B}-01)$ $0.244 \mathrm{E} 01(0.2948-01)$ $0.240 \mathrm{E} 01(0.322 \mathrm{E}-01)$ $0.240 E$ O $1(1(0.322 \mathrm{E}-01)$ 0.2308 O1( $0.373 \mathrm{E}-01)$ $0.217 \mathrm{E} 01(0.396 \mathrm{E}-01)$ $0.207801(0.4328-01)$ 0.178 or $0.4078-011$ $0.16580110 .3648=011$ $0.16580110 .3958-011$ 0.1658 011 $0.3658-011$ $0.169 E 0110.465 z-011$ 
ORNL/TM-5531

\section{INTERNAL DISTRIBUTION}
1. L. S. Abbott
2. R. G. Alsmiller, Jr.
3. C. F. Barnett
4. G. T. Chapman
5. J. F. Clarke
6. C. E. Clifford
7. G. de Saussure
8. J. K. Dickens
9. C. Y. Fu
10. H. Goldstein (Consultant)
11. D. J. Horen
12. W. E. Kinney
13. D. C. Larson
14. T. A. Love
15. F. C. Maienschein
16-25. G. L. Morgan
26. F. R. Mynatt
27. E. Newman

28. E. M. Oblow

29. R. W. Peelle

30. F. G. Perey

31. R. W. Roussin

32-35. RSIC

36. D. Steiner

37. M. L. Tobias

38. C. R. Weisbin

39. A. Zucker

40. P. F. Fox (Consultant)

41. W. W. Havens (Consultant)

42. A. F. Henry (Consultant

43. R. E. Uhrig (Consultant

44-45. Central Research Library

46. ORNL - Y-12 Technical Library

47. Laboratory Records, ORNL RC

48. ORNL Patent Office

49-50. Laboratory Records

\section{EXTERNAL DISTRIBUTION}

51. John D. Anderson, Physics Department, E. Division, Lawrence Livermore Laboratory, Livermore, CA 94550

52. D. Auton, Defense Nuclear Agency, Tactical Nuclear Division, Washington, DC 20305

53. H. H. Barschal1, Department of Physics, University of Wisconsin, Madison, WI 53706

54. R. C. Block, Nuclear Engineering \& Science Department, Rensselaer Polytechnic Institute, Troy, NY 12181

55. C. D. Bowman, Nuclear Sciences Division, RAD/P, National Bureau of Standards, Washington, DC 20234

56. R. E. Chrien, Physics Department, Brookhaven National Laboratory, Upton, NY 11973

57. C. L. Cowan, General Electric Company - BRDO, 310 de Guigne Drive, Sunnyvale, CA 94086

58. R. A. Dannels, Westinghouse Electric Corp., Nuclear Energy Systems, P. 0. Box 355, Pittsburgh, PA 15230

59. D. Dudziak, Los Alamos Scientific Laboratory, Los Alamos, NM 87544

60. R. L. Egli, Director, Reactor Division, ERDA, ORO

61. D. R. Finch, E. I. Dupont de Nemours \& Co., Savannah River Lab., P. 0. Box 117, Aiken, SC 29801

62. E. G. Fuller, B102 Radiation Physics Building, National Bureau of Standards, Washington, DC 20234

63. S. Gerstl, Los Alamos Scientific Laboratory, Los Alamos, NM 87544

64. R. C. Haight, Lawrence Livermore Laboratory, Livermore, CA 94550

65. J. Hardy, Bettis Atomic Power Laboratory, P. 0. Box 79, West Mifflin, PA 15122 
66. P. B. Hemmig, Division of Reactor Research and Development, ERDA, Washington, DC 20545

67. N. E. Holden, Knolls Atomic Power Laboratory, P. 0. Box 1072, Schenectady, NY 12301

68. R. J. Howerton, Lawrence Livermore Laboratory, Livermore, CA 94550

69. H. H. Hummel, Building 208, Room W-205, Argonne National Lab., Argonne, IL 60439

70. H. E. Jackson, Argonne National Laboratory, Argonne, IL 60439

71. M. Kalos, AEC Computing \& App. Math. Center, Courant Inst. of Math Sciences, New York Univ., 251 Mercer Street, New York, NY 10012

72. R. J. LaBauve, Los Alamos Scientific Laboratory, P. 0. Box 1663, Los Alamos, NM 87544

73. B. Leonard, Battelle-Northwest, P. 0. Box 999, Richland, WA 99352

74. A. E. Livolsi, Babcock \& Wilcox Co., Power Generation Division, P. 0. Box 1260, Lynchburg, VA 24505

75. C. R. Lubitz, G2-316, Knolis Atomlc Power Laboratory, P. 0. Box 1072, Schenectady, NY 12301

76. D. Mathews, Gulf General Atomic, P. 0. Box 608, San Diego, CA 92112

77. C. W. Maynard, University of Wisconsin, Madison, WI 53706

78. J. McCrosson, E. I. Dupont de Nemours \& Co., Savannah River Lab., P. 0. Box 117, Aiken, SC 29801

79. W. McElroy, Hanford Engineering Development Laboratory, P. 0. Box 1970, Richland, WA 99352

80. Michael S. Moore, P Division, Los Alamos Scientific Laboratory, Los Alamos, NM 87544

81. D. W. Muir, Los Alamos Scientific Laboratory, Los Alamos, NM 87544

82. R. J. Neuhold, Reactor Physics Branch, Reactor Development \& Technology, ERDA, Washington, DC 20545

83. H. W. Newson, Dept. of Physics, Duke Univ., Durham, NC 27706

84. V. J. Orphan, Science Applications Inc., P. 0. Box 2351, La Jolla, CA 92037

85. N. C. Paik, Westinghouse Electric Corp., Advanced Reactor Div., Waltz Mill Site, P. 0. Box 158, Madison, PA 15663

86. S. Pearlstein, National Neutron Cross Section Center, Brookhaven National Laboratory, Uptom, NY 11973

87. E. M. Pennington, Building 208, Argonne National Laboratory, 9700 S. Cass Avenue, Argonne, IL 60439

88. Enloe T. Ritter, Nuclear Science, Division of Physics Research, ERDA, Washington, DC 20545

89. G. L. Rogosa, Assistant Director for Nuclcar Sciences, Division of Physical Research, ERDA, Washington, DC 20545

90. Robert Schenter, Hanford Engineering Development Laboratory, P. 0. Box 1970, Richland, WA 99352

91. R. Sher, Dept. of Mechanical Engineering, Stanford University, Stanford, CA 94305

92. A. B. Smith, Argonne National Laboratory, Argonne, IL 60439

93. J. R. Smith, Aerojet Nuclear Company, P. 0. Box 1845, Idaho Falls, ID 83401 
94. Leona Stewart, Los Alamos Scientific Laboratory, Los Alamos, NM 87544

95. Bruce Twining, Systems and Applications Studies Branch, Division of Controlled Thermonuclear Research, ERDA, Washington, DC 20545

96. W. H. Walker, Applied Mathematics Branch, Chalk River Nuclear Laboratory, Atomic Energy of Canada, Ltd., Chalk River, Ontario, Canada

97. J. M. Williams, Division of Controlled Thermonuclear Research, ERDA, Washington, DC 20545

98. P. G. Young, Los Alamos Scientific Laboratory, Los Alamos, NM 87544

99. Research and Technical Support Division, ERDA, ORO

100-126. Technical Information Center, Oak Ridge 